\title{
Incorporating Computational Fluid Dynamics Into The Preliminary Design Cycle
}

Jonathan Knighton Shelley

Brigham Young University - Provo

Follow this and additional works at: https://scholarsarchive.byu.edu/etd

Part of the Mechanical Engineering Commons

\section{BYU ScholarsArchive Citation}

Shelley, Jonathan Knighton, "Incorporating Computational Fluid Dynamics Into The Preliminary Design Cycle" (2005). Theses and Dissertations. 631.

https://scholarsarchive.byu.edu/etd/631

This Thesis is brought to you for free and open access by BYU ScholarsArchive. It has been accepted for inclusion in Theses and Dissertations by an authorized administrator of BYU ScholarsArchive. For more information, please contact scholarsarchive@byu.edu, ellen_amatangelo@byu.edu. 
INCORPORATING COMPUTATIONAL FLUID DYNAMICS INTO THE

PRELIMINARY DESIGN CYCLE

\author{
By \\ Jonathan Knighton Shelley \\ A thesis submitted to the faculty of \\ Brigham Young University \\ in partial fulfillment of the requirements for the degree of
}

Master of Science

Department of Mechanical Engineering

Brigham Young University

August 2005 
Copyright (C) 2005 Jonathan Knighton Shelley

All Rights Reserved 
BRIGHAM YOUNG UNIVERSITY

GRADUATE COMMITTEE APPROVAL

\author{
of a thesis submitted by \\ Jonathan Knighton Shelley
}

This thesis has been read by each member of the following graduate committee and by majority vote has been found satisfactory.

Date

Date

Date
C. Greg Jensen, Chair

Kenneth W. Chase

Spencer P. Magleby 


\section{BRIGHAM YOUNG UNIVERSITY}

As chair of the candidate's graduate committee, I have read the thesis of Jonathan Knighton Shelley in its final form and have found that (1) its format, citations, and bibliographical style are consistent and acceptable and fulfill university and department style requirements; (2) its illustrative material including figures, tables, and charts are in place; and (3) the final manuscript is satisfactory to the graduate committee and is ready for submission to the university library.

Date

Accepted for the Department
C. Greg Jensen

Chair, Graduate Committee
Matthew R. Jones

Graduate Coordinator

Accepted for the College
Alan R. Parkinson
Dean, Ira A. Fulton College of Engineering and Technology




\begin{abstract}
INCORPORATING COMPUTATIONAL FLUID DYNAMICS INTO THE PRELIMINARY DESIGN CYCLE

\author{
Jonathan Knighton Shelley \\ Department of Mechanical Engineering \\ Master of Science
}

Industry is constantly looking for ways to bring new or derivative products to market in the shortest amount of time for the least amount of money. To accomplish this, Industry has adopted Computer Aided Engineering (CAX) tools that perform structural, flow, manufacturing, and cost analysis. The way in which a company utilizes these CAX tools can determine the success of these new products.

One of these tools that Industry often struggles with in the preliminary design of a product is Computational Fluid Dynamics (CFD). Some of the challenges presented by CFD are the time it takes to create a CAD model, generate a valid grid for analysis, obtain a solution, post-process results, and review the result. The objective of this thesis is to develop an approach that will reduce the time it takes to go from a concept to a 
solution ready for review. This approach, addresses how to first, build CAD model for use in downstream applications, second, automate the grid generation process, and third, automate the post process and documentation of the results.

Using this approach a conceptual study of a two stream mixing problem was performed. The approach showed that the creation of the first model took about twenty percent longer than the standard practice used in industry today. However, once the first model was completed, different concepts could be added to the CAD model and be ready for analysis in less than half the time when compared to standard practices. This time savings can then be used to explore more concepts. After each model was analyzed, it was post-processed using an automated script. With the post-processed results the Design Review Tool (DRT) was developed to automate the documentation of the results. Using the DRT each post-processed case was organized into a web page and saved for review in less than five seconds.

This approach will enable the aerospace, automotive, and other industries to use CFD to more effectively explore the design space in the development of new and derivative products. This research demonstrates the process to reduce the time required to go from CAD-to-Grid, postprocess the results, and create the documentation needed to develop new products. 


\section{ACKNOWLEDGMENTS}

I would like to first thank my wife and children for their love and support.

Without their support this never would have happened. Second, I want to thank Dr. C.

Greg Jensen, Dr. Stephen Kramer, and the BYU faculty for their encouragement, friendship, help, and support throughout my graduate studies. Lastly I want to thank Pratt and Whitney for supporting this research. 


\section{TABLE OF CONTENTS}

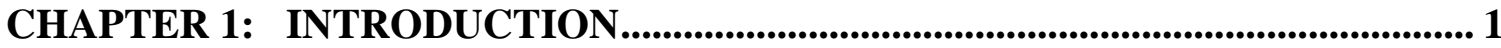

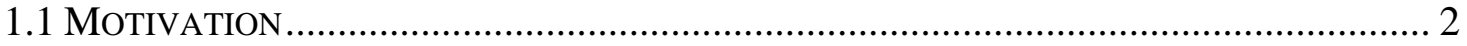

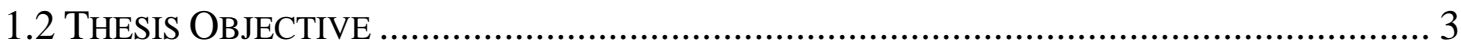

1.3 DeLIMITATION OF THE PROBLEM ………………................................................ 4

CHAPTER 2: LITERATURE REVIEW: ............................................................... 5

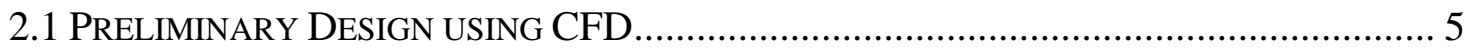

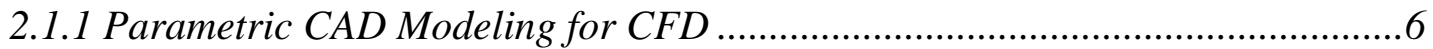

2.2 LINKING COMMERCIAL CAD WITH CAE TOOLS........................................................ 6

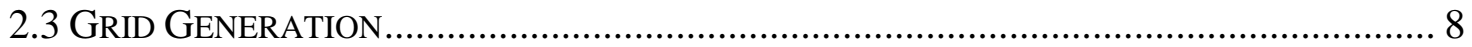

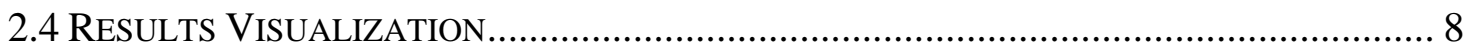

CHAPTER 3: METHOD ……........................................................................................ 11

3.1 CAD Modeling FOR CFD IN PRELIMINARY DESIGN …………………………....... 11

3.1.1 Construct a Master Cross Sectional Sketch (MCSS) ........................................12

3.1.2 Model the Component Using the MCSS References ..........................................13

3.1.3 Assembly of the Various Components..............................................................14

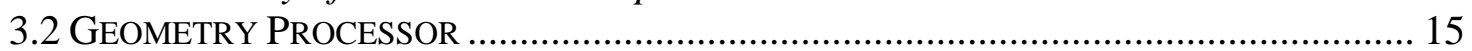

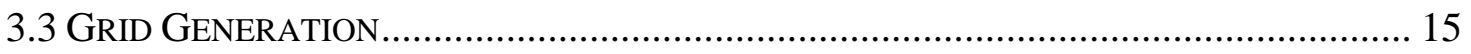

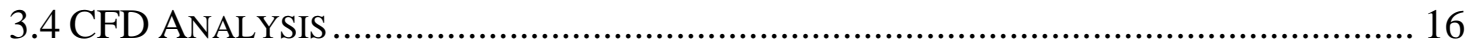

3.4.1 Defining the Boundary Conditions ..............................................................16

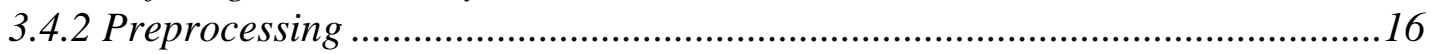

3.4.3 Analyzing the Model ...................................................................................17

3.4.4 Post Process Results ..................................................................................17

3.5 DESIGN REVIEW TOOL ………………......................................................... 17

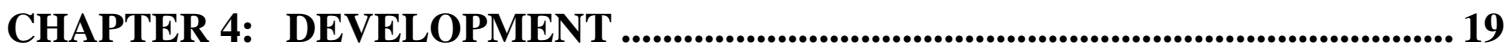

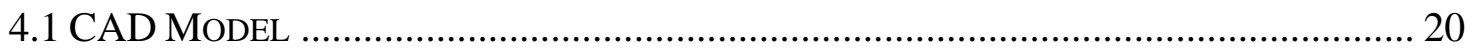

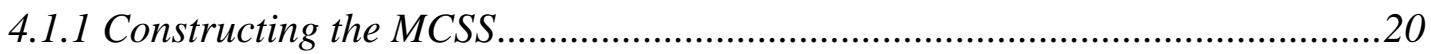

4.1.2 Component Modeling.................................................................................23

4.1.3 Assembly Modeling ......................................................................................2

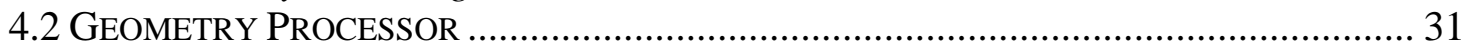

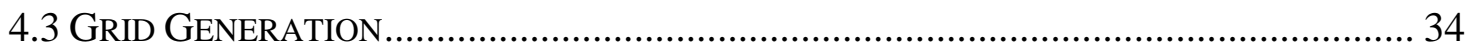

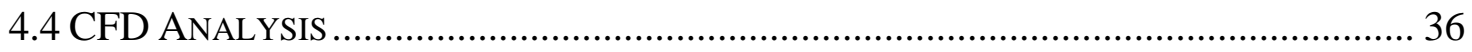

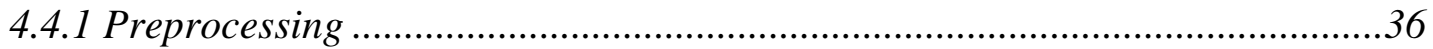

4.4.2 Boundary Conditions ....................................................................................

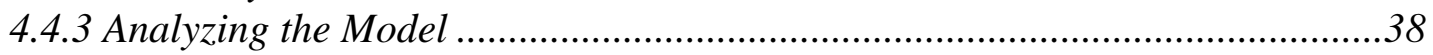

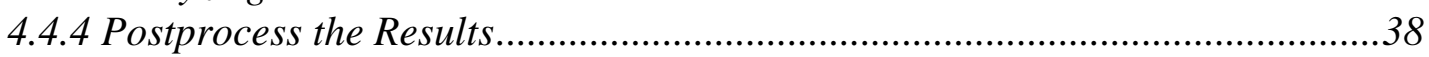

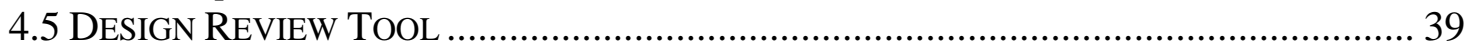


CHAPTER 5: DISCUSSION OF RESULTS

5.1 Results: Automated Grid Generation of Parametric Models .................... 49

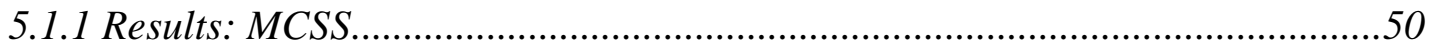

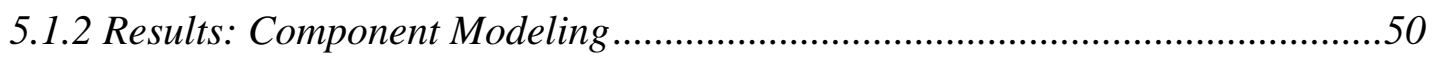

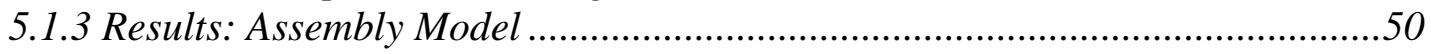

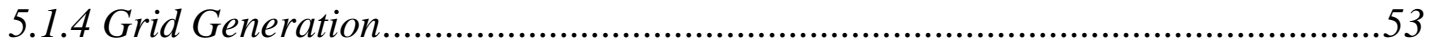

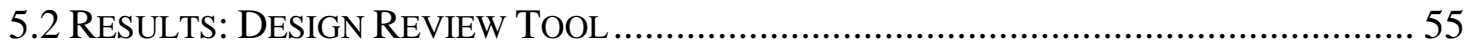

5.2.1 Does the DRT present the results effectively? .............................................56

5.2.2 Are the results presented in a timely manner?..................................................56

5.2.3 Are results of each case setup to be archived for later use? ............................56

CHAPTER 6: CONCLUSIONS ...................................................................................... 59

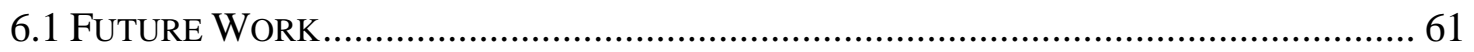

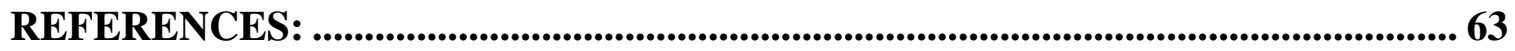

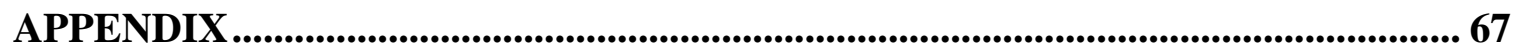

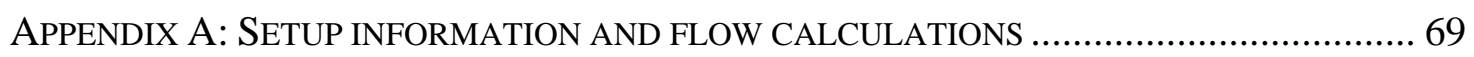

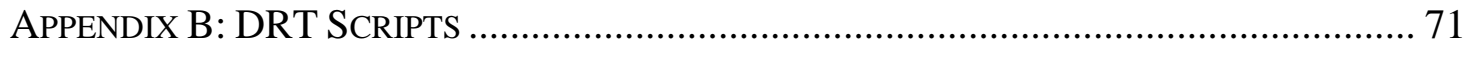

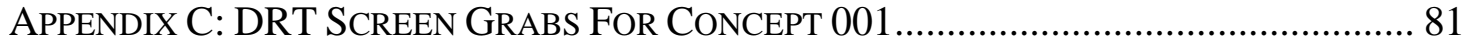




\section{LIST OF FIGURES}

Figure 3-1 Elements for incorporating CFD into preliminary design........................ 11

Figure 3-2 Defining a master cross sectional sketch ........................................... 12

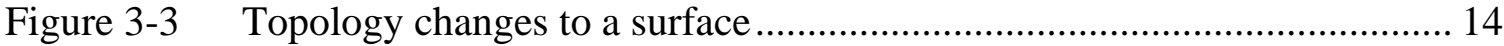

Figure 4-1 Rolls-Royce Trent combustor (Cervenka 2000) ..................................... 19

Figure 4-2 Master Cross Section Sketch................................................................... 21

Figure 4-3 Entities referenced from the MCSS for the front-end concepts ................. 21

Figure 4-4 Entities referenced from the MCSS for the ID and OD liner concepts...... 22

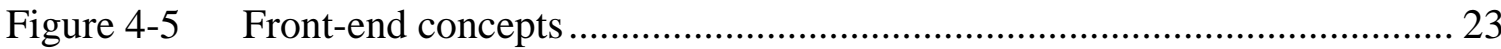

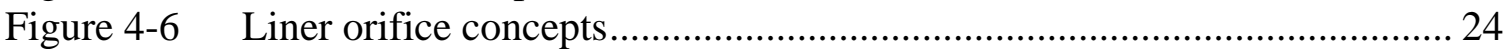

Figure 4-7 Before and after the model was trimmed ................................................ 24

Figure 4-8 Surfaces tagged for mesh generation. ..................................................... 26

Figure 4-9 Family, faces, and curves tagged for front-end concept \#3 ....................... 27

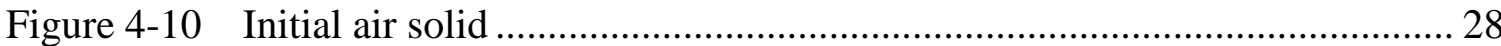

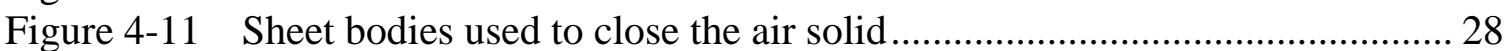

Figure 4-12 Air solid model..................................................................................... 29

Figure 4-13 Assembly issues when a component is toggle off and another one is

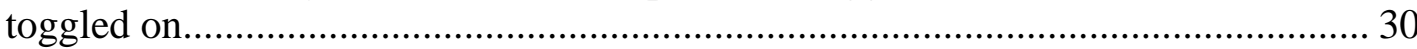

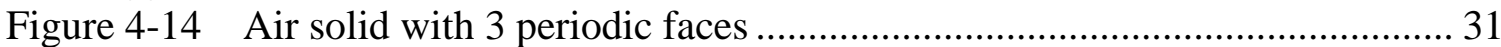

Figure 4-15 With and without running the geometry processor ................................... 34

Figure 4-16 Flow velocities into the plenums.......................................................... 37

Figure 4-17 A screen grab of the preliminary CFD study page ................................. 41

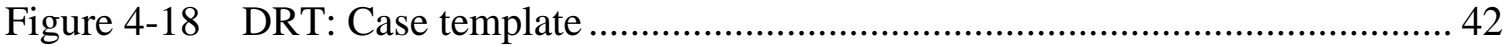

Figure 4-19 DRT: Model section ........................................................................... 43

Figure 4-20 DRT: Grid section ............................................................................ 43

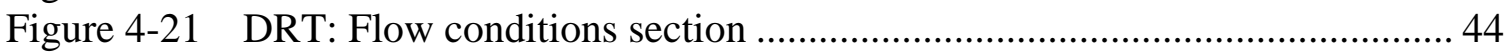

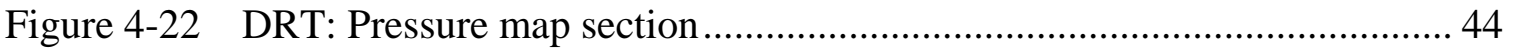

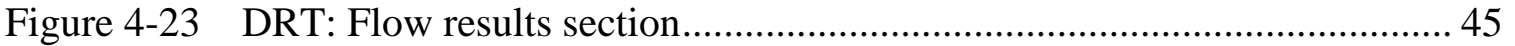

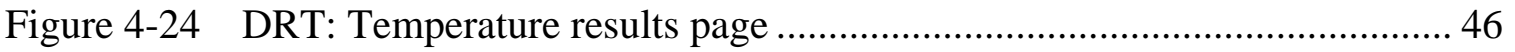

Figure 4-25 DRT: Temperature slice information image .......................................... 46

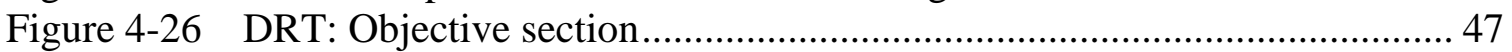

Figure 5-1 Base assembly with front-end concept \#1 and liner hole concept \#3 ........ 51

Figure 5-2 Case 002 air solid assembly with front-end concept \#2 and liner hole

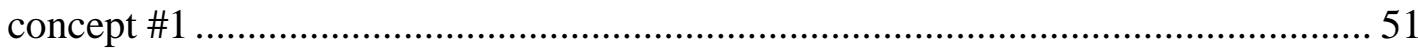

Figure 5-3 Case 006 air solid assembly with front-end concept \#3 and liner hole

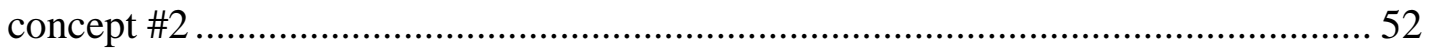

Figure 5-4 ICEM screen capture of the input file that was imported from

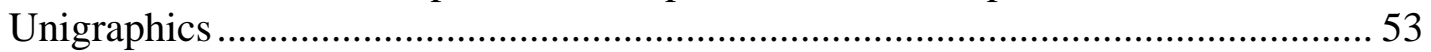

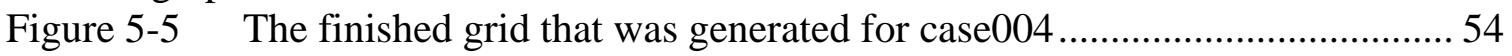




\section{CODE FRAGMENTS}

Code Frag 4-1 Code used to open the CAD model, read in the expression file and

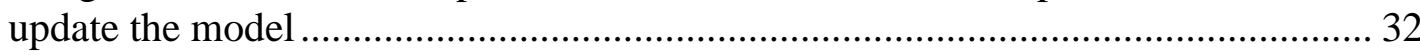

Code Frag 4-2 Find the specified family edges and add points and tag them ............... 33

Code Frag 4-3 Script created to automate the grid generation process ......................... 35

Code Frag 4-4 Code to import geometry from Unigraphics and add density

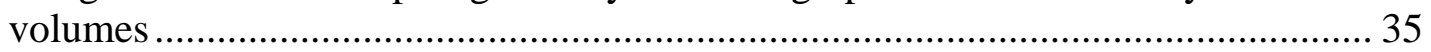

Code Frag 4-5 Ensight commands to read in the model and results............................. 38

Code Frag 4-6 Input needed to define the location of the files for the DRT ................. 39

Code Frag 4-7 Model class module for the DRT .......................................................... 40 


\section{INTRODUCTION}

Today’s highly competitive market place demands high quality, low-cost

products, developed in a timely, rapid fashion. According to Ulrich and Eppinger (2001), effective product development and release requires: planning, concept development, system-level design, detailed design, testing and refinement, and production ramp-up. Conceptual and preliminary design steps define product discriminators and provide improved quality and lower cost. Automated best-in-class conceptual and preliminary design tools facilitate these steps.

Historically companies have been unable to explore all possible concepts and combinations of concepts due to limited resources and competitive pressures. Concepts have and are being carried forward to the detailed design phase based on partial information, partial exploration of the design space, and insufficient development. For most companies still challenged by limited resources and pressures from their competition, their detailed design phase is littered with technical challenges and roadblocks caused by premature termination of the conceptual and preliminary design phases.

Ideally, all possible concepts would be explored before selecting the one that best meets requirements and is deemed ready for detailed design and production. Tools like computer-aided design (CAD), finite element analysis (FEA), and computational fluid dynamics (CFD), provide simulations that are used to numerically compare concepts and help the engineer understand the problems and challenges of particular concepts. 
However, Haimes (2003) states, and personal experience in industry confirms, that getting the first CAD model(s) ready for analysis tends to take weeks to months. CAD models are being integrated with CAE tools as faster and more capable computers become available (Alonso et al. 2003). Limiting factors for these integrated design tools becomes user knowledge, modeling techniques, software capabilities, and computer hardware resources.

\subsection{Motivation}

Today, in industry a fluids engineer works with the designer to create an air solid for a given concept. Typically a cross section is given to the designer to define the 2Dimensional shape. The cross section is commonly unparameterized. Using this unparameterized cross section as a reference the designer creates the air solid. When the air solid is completed, the designer passes the model to the grid specialist. The grid specialist looks over the model and generates the first grid. If there are problems, i.e. leaks, misalignment of surfaces, slivers, etc, the designer is asked correct the model. The designer then changes the model and gives it back to the grid specialist. The grid specialist then creates the final grid. If the fluids engineer wants to run variants of the model or explore different topology concepts, he works with the designer to create the new air solids. This can take anywhere from a few hours to a few weeks. The air solids are then handed to the grid specialist and depending on the changes, it takes between a few hours to a week to generate each new grid. This current process is time consuming, costly, and involves multiple people.

To better utilize the array of modern CAD/CAE tools and the company's computing resources a methodology is needed, which reduces the time required to go 
from CAD/to/Mesh. This timesaving could then be used to explore more concepts before having to select a particular design to carry forward to the detailed design phase.

Another challenge to using CFD in conceptual and preliminary design is the amount of data that must be processed and presented. Currently, the standard practice in industry is to post process the CFD solution and generate images for a set of parameters. The analyst then proceeds to make a presentation. The presentation consists of a discussion of the model, a review of the boundary conditions, and pressure field, and then a review of the results such as temperature, velocities, etc. If performed manually, the time it takes to document each case can take hours. When iterations of the model are performed, the analyst commonly takes the previous presentation as a template and updates it. This process of updating the presentation results in delaying the review of the results until a formalized presentation can be made. A method is needed which will allow data to be processed in a consistent manner and formatted for quick review.

\subsection{Thesis Objective}

The focus of this thesis will be to develop a method that integrates the parametric capabilities of CAD systems with CFD analysis tools in order to solve some of the challenges discussed above. Solving these challenges will enable the conceptual and preliminary design teams to evaluate significantly more concepts in a timely fashion.

Once the method is proven, the author believes that the geometric complexity, quantity of engineering knowledge embedded and the frequency of tool reuse will naturally increase.

To illustrate the methodology developed in this thesis a two stream mixing problem will be analyzed. The following specific questions will be addressed: 
1. Can valid CFD grids be made in an automated way from parametric models that undergo predictable topology changes?

2. Can valid CFD grids be made in an automated way when one concept is replaced with a different concept in an assembly?

3. How can CFD data be evaluated more efficiently for design reviews?

4. How can CFD data be effectively documented for later use?

The CAD models will be created using Unigraphics (UG). ICEMCFD commonly referred to as ICEM, will be used to create the grids required for the CFD analysis package. The CFD solver will be Pratt and Whitney’s in-house code All-Star. Ensight visualization software will be used process the CFD data. To link the various tools together, as well as format the data for future use, the scripting language, Python will be used. It is anticipated that by answering these questions, design teams in industry will be able to explore more concepts using CFD before a concept is selected and moved into the detailed phased.

\subsection{Delimitation of the Problem}

The author believes that the method presented in this work is general enough to be applied to the various commercial CAD and CAE tools. However, for this thesis only the tools mentioned above will be considered. Every attempt will be made to specify loads, boundary conditions, constraints, etc. so that the CFD model will conform to industry practices. 


\section{LITERATURE REVIEW:}

Research work and topics that form the foundation on which this research will build are: preliminary design using CFD, parametric modeling for CFD, linking commercial CAD to CAE tools, grid generation, and presentation of results. In this prospectus a brief summary is given as to current work that supports the direction of this thesis. Where possible, I have included quotes that support and encourage the direction being pursued.

\subsection{Preliminary Design using CFD}

CFD modeling of complex geometry in the aerospace industry is commonly used in the design process (Crocker et al.1998). Incorporating CFD modeling of assemblies into the preliminary design process presents challenges due the time required to generate the first grid and subsequent grids. Grid generation ”is the most labor intensive and time consuming part of computation aerosciences” (Samareh 1999). Haimes et al. (2003) states that the time to go from CAD to generating the first CFD case can take weeks to months. The bulk of the time and labor is spent simplifying the geometry, exporting the model to the CAE tool, repairing the geometry if needed, and tagging the model for gridding. For these reasons Smith (1996) states that research is needed to eliminate grid generation time so that more time can be used to incorporate analysis more fully into the concept generation stage. 


\subsubsection{Parametric CAD Modeling for CFD}

Parametric CAD systems use a "feature tree" approach to construct the solid model. Each feature created is updated in the order that it was created. Features consist of sketches, lines, curves, points, surfaces, solids, etc. Using these features in a tree structure enable the solid model to update easily and have good reusability characteristics (Hoffmann 2001).

CAD is the enabling technology for the creation of solid models, design changes, and down stream analyses (Lee 1999). Ault (1999) states that the development of the CAD model is critical to maintain design intent. Today most engineering analyses use parametric solid model(s) as the foundation for the analysis models (Srinivassen et al. 2001). If a model is built parametrically, design changes can be easily performed (Anderl 1995). The parametric model can be modified by either manually modifying the expressions or by reading in an expression file (Rohm et al. 2000).

\subsection{Linking Commercial CAD with CAE Tools}

Integration of CAD tools to downstream CAE applications becomes an enabler to improving the product development process (Armstrong 2002). Today, there are two approaches to transferring the data from the CAD system to the CAE tool. They are by neutral file format (i.e. STEP and IGES) or direct translators. Krause et al. (1997) discusses an approach to working with neutral formats and how to repair errors caused during the translation in the geometry. If the model has been translated using a neutral file format, the user is required to assign entity parameters to each surface and curve. This method is fine if the model will only be gridded once. 
Direct translators provide a one directional link from the CAD model to the CAE tool. This integration of the CAD and CAE tools for passing complex geometry from the CAD tool to different CAE tools in a reliable and timely fashion has only been available for the past few years (Dawes et al. 2000, Tangirala et al. 2000). Today's direct interfaces allow the "generation of complex-geometry grids without requiring boundary conditions and mesh sizes to be respecified” (Malecki et al. 2001). They do this by storing the necessary information needed for the grid generation phase in object attributes. Subrashekar et al. (1995) said attributes are "ideal candidates to store information relevant to a particular phase in the life-cycle of a product. For EXAMPLE: design, analysis, assembly, process planning, etc.”

However, one of the current limitations to using a direct translator in an automated way is that parametric changes to the geometry can result in topological changes (Hardee et al. 1999). These topological changes can result in new objects that have not been assigned. This will result in an invalid grid when the model is translated and gridded. To address some of these issues, King (2004) developed a tool that would look at feature arrays and User Defined Features (UDF's) and apply the attributes from the parent feature in the array to all of the instances. While this addresses one set of topology problems, it does not address problems such as a holes on a periodic plane or surface that are broken in two resulting in four edges for the hole instead of two, or a hole that lies on a subdivided face resulting in eight edges instead of four. This thesis will look at addressing the topology changes mentioned above that are not part of an array. 


\subsection{Grid Generation}

Crocker (1998) states that "generating a quality grid in a reasonable amount of time” is a significant challenge. Usually, the CAD model has already been designed. For the model to be used in CFD it generally needs simplification. This requires suppressing holes, rounds, chamfers, etc. These simplifications are often performed in the CAD package due to the poor modeling capabilities of the gridding tool. For complex geometries the common gridding technique used is an unstructured grid. Unstructured grids enable the gridding process to become more automated because "they are capable of resolving irregularly shaped domains more easily and with greater efficiency” (Newman et al. 1999). Newman further states that unstructured grids can be "adapted and locally enriched where needed without affecting other regions of the mesh.” Although unstructured grids provide the flexibility to grid all of these irregular shapes, some geometry contains problem spots that require hand fixing. This need for hand fixing prevents the process to be fully automated.

\subsection{Results Visualization}

As computer tools evolve, they enable the designers to generate more and more data. Hogge (2002) states, that after a design study has been performed it can be challenging to sort thru all the data. Most of these visualization tools have two or three ways for the engineer to get at the data. They are the GUI, macros, and API’s (Hogge, 2002). For small concept studies or preliminary design projects API are poorly suited due to the development time required versus an interactive approach and the developer must know the API of the particular system being used (Rohm, 2001). For these reasons, this 
research looks to make use of macros to processes the visualization data due to the ease in creating them for small conceptual studies. 


\section{METHOD}

The following paragraph describes the union of various commercial and noncommercial CAx tools. Currently, the CFD process is manual, error-prone, and too time intensive for use in preliminary design of complex models. While no significant research and development is added to the commercial and noncommercial tools themselves, this work will focus on the development of interfacing the different tools. The following approach will describe in detail the steps that will be used to handle some topology changes as well as replacing components in the assembly model which goes beyond the common parametric changes to an air-solid used in industry today.

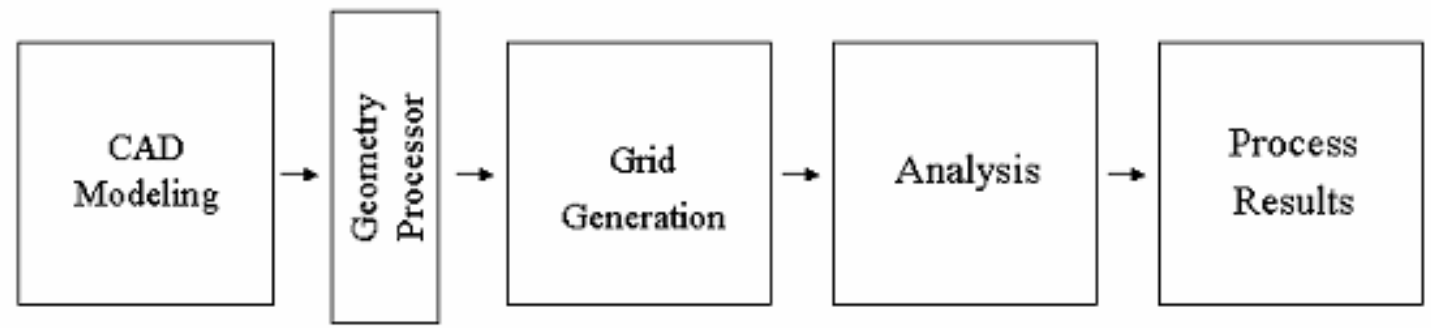

Figure 3-1 Elements for incorporating CFD into preliminary design

\subsection{CAD Modeling for CFD in Preliminary Design}

The CAD modeling strategy can be summarized in three steps;
a. Construct a Master Cross Sectional Sketch (MCSS)
b. Model the component using the MCSS references
c. Assembly of the various components 
If these steps are followed in the consistent and standard way as disclosed below the result will be a robust and reusable model.

\subsubsection{Construct a Master Cross Sectional Sketch (MCSS)}

The purpose of the MCSS is to define key references and features for an assembly. Before a MCSS can be constructed, these key references and features (i.e. Datum planes and axes, Assembly features, Component locations, etc) need to be defined. Below is an example of using datums to define the location of square box and cylinder in an assembly using a MCSS.

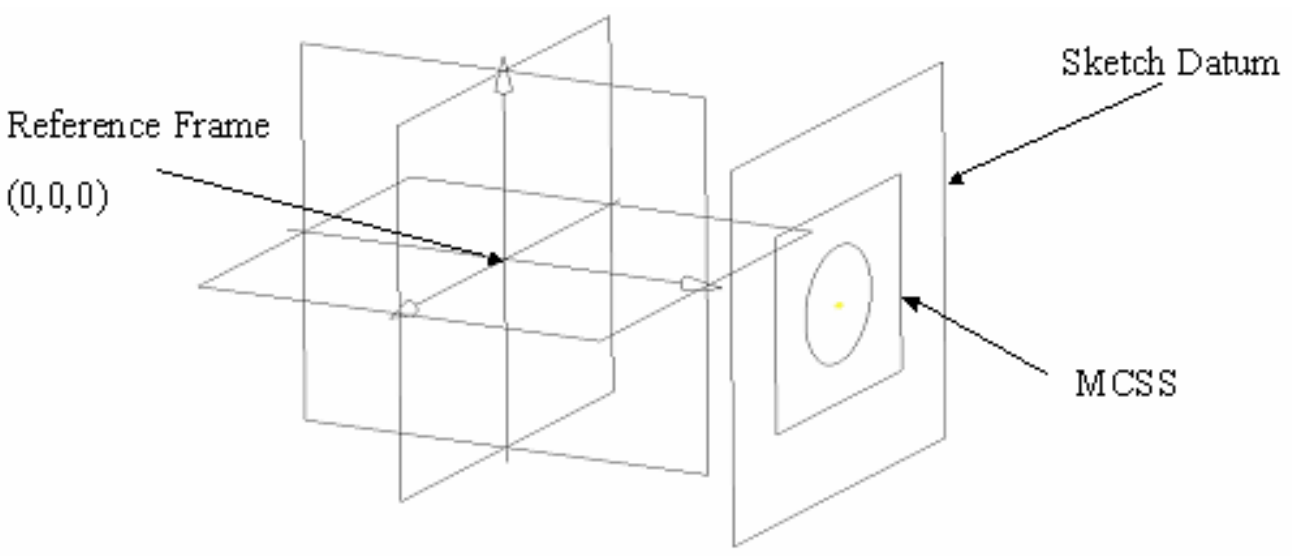

Figure 3-2 Defining a master cross sectional sketch

In the figure above there are four datum planes, three datum axes and one sketch. The datum plane with the sketch of the square and the circle would be an assembly plane or interface where two components would be positioned. By using common datums in the MCSS to locate the different components reduces the time required to create the assembly. Also, changes in the MCSS will propagate through the entire assembly in a consistent manner. 
Once the master sketches are created, the designer(s) can work off the same sketches to make certain that the interface points and locations are consistent. This guarantees that the components will match up properly when it comes to populating the assembly and eliminate unintentional gaps.

\subsubsection{Model the Component Using the MCSS References}

In order for the components to take advantage of the information stored in the MCSS Inter-part geometry and Inter-part dimensioning will be used. Inter-part geometry linking provides a way for geometry defined in the MCSS to be referenced by the different components. The linked geometry enables the various components to be adjusted to changes that occurred in the MCSS. If used properly, inter-part geometry linking helps to ensure that the overall assembly will maintain design intent during design iterations.

Inter-part dimensioning allows information from the MCSS to flow to the individual components. This is done by creating assembly level parameters in the MCSS and linking them to the individual components. For example, if the overall length of the square and the circle defined in the example above were to be ten inches long, parameters such as

$$
\begin{aligned}
& \text { Total_length }=10 \\
& \text { Box_length }=\text { Total_length } / 3 \\
& \text { Cylinder_length }=\text { Total_length-Box_length }
\end{aligned}
$$

can be defined. Parameters such as these will be defined in the MCSS and referenced by the components to define their critical dimensions.

Once the component is modeled, attributes needed for mesh generation will be assigned to the different geometric entities. Defining the element size for each face and 
curve often requires a few iterations before the different features are properly resolved. When the analyst is satisfied with the grid, this information can then be used for subsequent designs. However, when the topology is changed the analyst needs to recognize the topological changes, tag any new geometric entities, and ensure the new entities will be properly resolved. For example, the top face on the left block (below) needs nine entities to properly resolve its shape, while the right block requires an additional edge to resolve its top face.

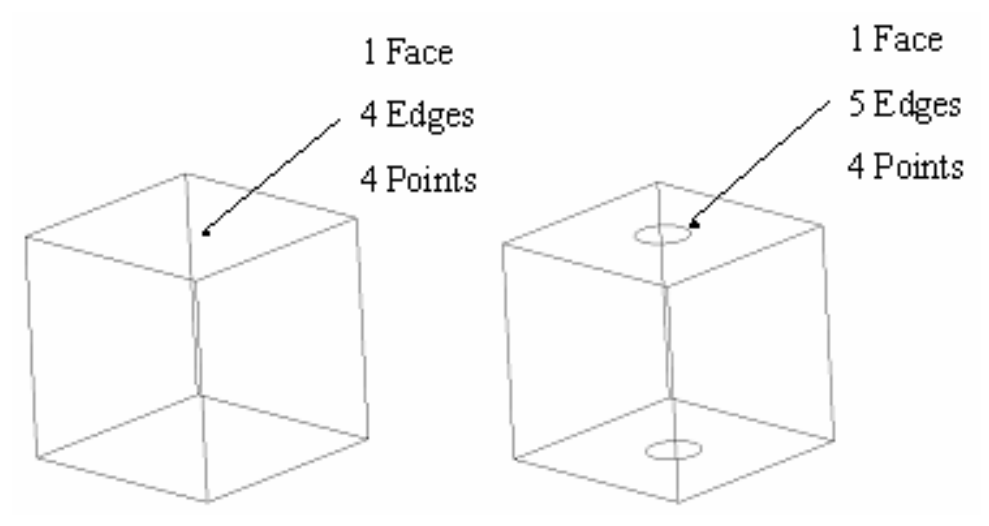

Figure 3-3 Topology changes to a surface

\subsubsection{Assembly of the Various Components}

Once the components are modeled, they will be added to the assembly. If the component modeling step was performed correctly, the various components should require little to no information in order to be assembled correctly. At the assembly level, interface surfaces may be needed to close any gaps between the various components. These interface surfaces may be tied to expressions in order to toggle them on or off , based on a particular configuration. 
Assembly expressions will be used to toggle the parametric components on/off as well as any interface surfaces. This will allow an input file to modify the assembly in order to represent the different concepts.

\subsection{Geometry Processor}

The geometry process step is used to sort through the model looking for any entities, such as edges, that are not currently tagged and assign attributes as needed. For predetermined topological changes, such as the one in Figure 3-3, the geometry processor will need to be able to recognize the new topology and tag the appropriate entities. The geometric process will need to do the following:

1. Collect the attribute names that may contain topology changes

2. Modify the expressions and update the CAD model

3. Search the entities for specified attributes names

4. Check entities to see if they are tagged

5. Assign the proper attributes to any new topology

6. Save the model

\subsection{Grid Generation}

ICEM CFD will be used to create the grid. The first grid will be created interactively to determine the steps required. Each step will be recorded so that the steps can be incorporated into a script. This script will then be used to perform the following steps necessary for gridding.

1. Adding density volumes

2. Generating the grid

3. Smoothing the grid 
4. Saved the grid for review.

The review step is necessary to verify and fix the grid, if needed, before it is preprocessed.

\subsection{CFD Analysis}

\subsubsection{Defining the Boundary Conditions}

Boundary conditions are the inputs required to solve the differential equations used in CFD analysis. Some common boundary condition types are inlet speeds, walls, periodic faces, symmetry planes, etc. Initial boundary conditions must be set before the model is solved. These boundary conditions can then be modified to match the desired conditions. Once these conditions are set they usually require minor changes, if any, for the subsequence derivative models.

When performing a CFD analysis, there are three steps that are required in order to obtain the results. The first step is preprocessing the grid

\subsubsection{Preprocessing}

For this thesis, standard practices were used for the preprocessing. These standard practices include checking the grid for errors, assigning the boundary conditions to the nodes, and breaking up the model to be run on multiple computers. When the grid is ready, the next step is analyzing the model 


\subsubsection{Analyzing the Model}

As stated in the introduction, the focus of this thesis is on the model preparation process and not the analysis results. Since no experimental data is available to validate the results, CFD results will be assumed to be valid and provide evidence that the model preparation is adequate. When the CFD solver has converged to a solution the final step is to post process the results.

\subsubsection{Post Process Results}

Ensight visualization software was used to perform the post processing for each converged solution. To automate this step a command file (macro) must be created for the first case. In general the command file will need to

1. Open the analysis model

2. Load the results file

3. Calculate desired variables for study

4. Generate images of the model

5. Output the data

6. Exit the program

Once the macro file is setup, it will perform all of the subsequent postprocessing in a consistent way.

\subsection{Design Review Tool}

The Design Review Tool (DRT) is created to organize the results gathered from the post processing step in a timely fashion. It also formats the results for later use. To do this, a standard file structure is required to allow the DRT to organize the data into a set 
of web pages that can be easily reviewed by other design team members. The web pages contain

1. Images of the concepts

2. Tables containing flow conditions and results

3. Graphs displaying the results

By presenting this data effectively in an automated fashion, the time required to prepare a design review should be significantly reduced. It also enables the design team to look at the results as soon as they are available as well as storing them for later use. 


\section{DEVELOPMENT}

The method presented in chapter 3, as shown in Figure 3-1, was applied to a two stream mixing problem. One example from industry of a two stream mixing problem is a combustor in a gas turbine engine shown in Figure 4-1. As shown the fuel spray nozzle injects the first stream or hot stream. The holes in the liners allow the injection of the second stream or cold stream.

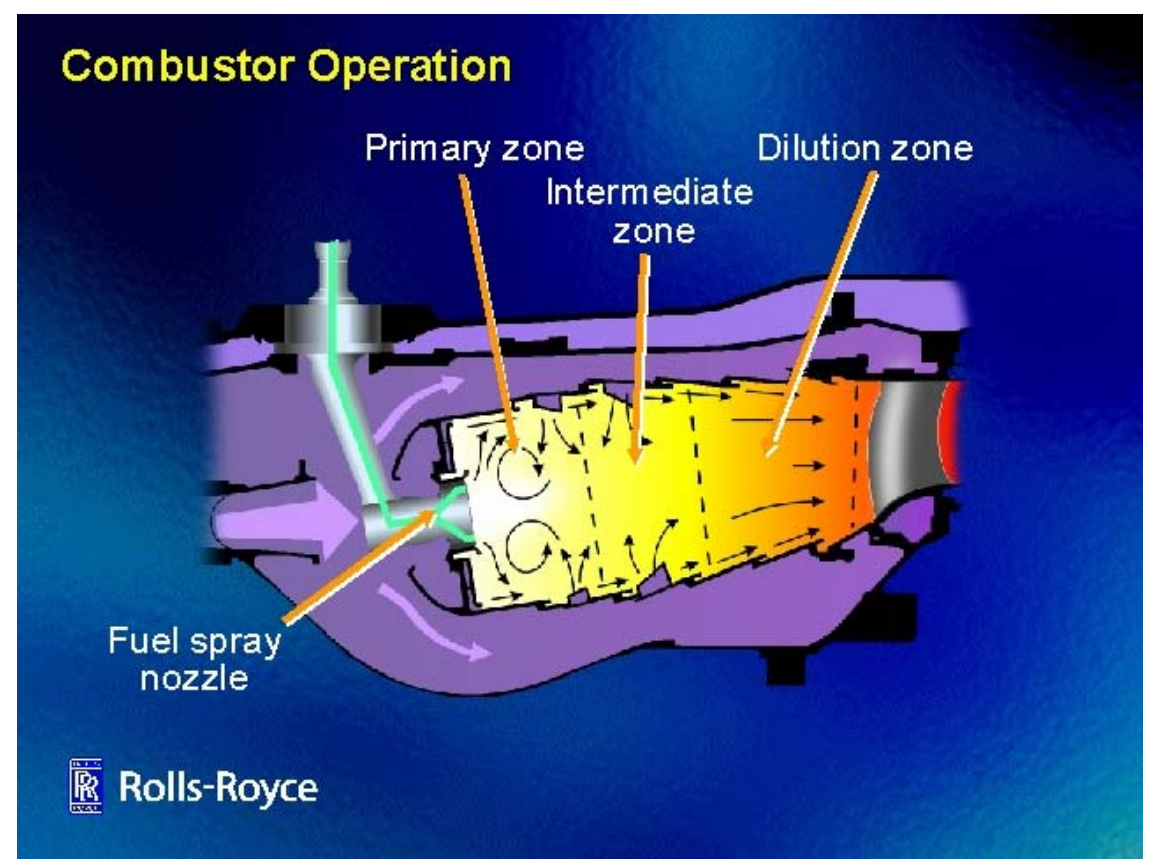

Figure 4-1 Rolls-Royce Trent combustor (Cervenka 2000)

For this study, a simplified representation of a combustor was used. It consists of three different components. These components are a front-end, which introduces the hot 
stream and the outer and inner liners, which introduces the cold stream. Different concepts and configurations were explored for each component to determine which concept provided the most uniform temperature at the exit plane.

\subsection{CAD Model}

The foundation for the two-stream mixing problem is the CAD model. The following sections present the steps used for the creation of the components and assembly.

\subsubsection{Constructing the MCSS}

For the two stream mixing problem, a MCSS was created. In the MCSS the envelope of the assembly, datum references, and assembly interfaces are specified. First, to define the origin, three datum planes were created. Second, to define the centerline of the two-stream mixing problem a datum axis was created. Third, the assembly envelope, containing the mixing chamber and the OD and ID plenums was laid out. Fourth, the injection location for the second stream of air was added. The MCSS for the simplified two stream mixing problem can be seen in Figure 4-2. 


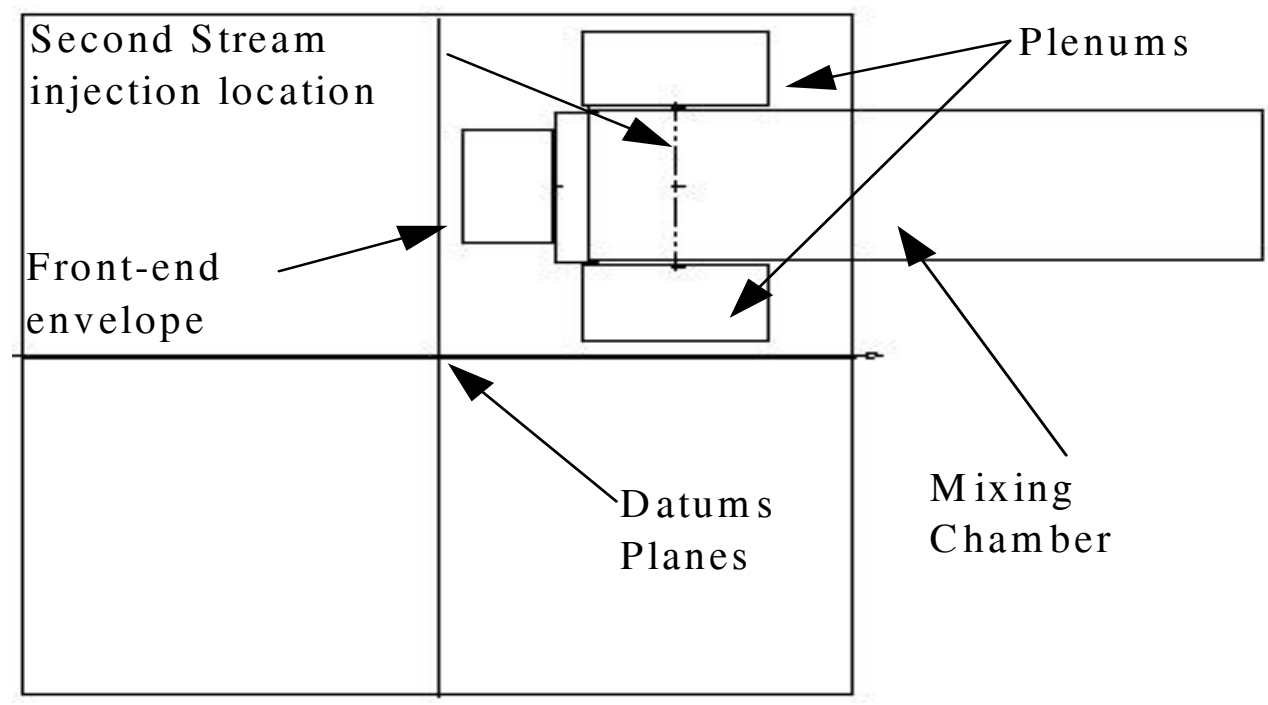

Figure 4-2 Master Cross Section Sketch for the two stream mixing problem

From the MCSS, the different components reference the necessary entities. The datums, lines and point that are referenced by the front-end concepts are shown in Figure 4-3.

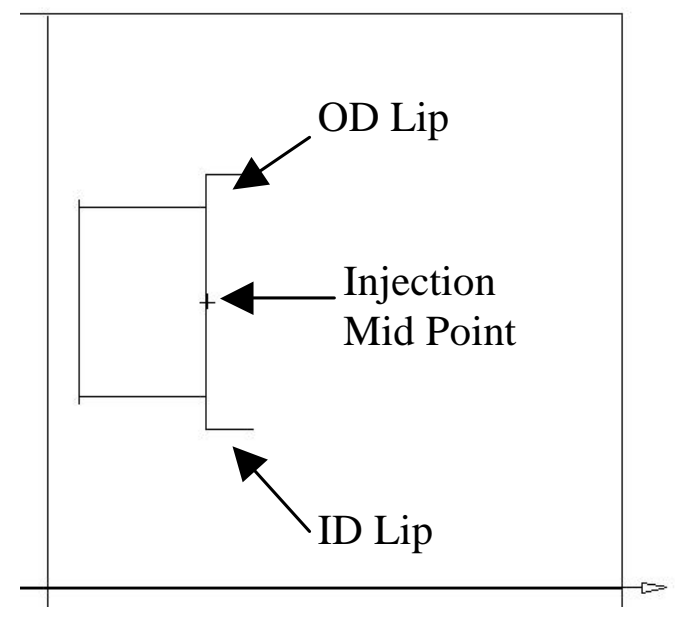

Figure 4-3 Entities referenced from the MCSS for the front-end concepts 
The outer and inner lips indicate where the front-end mates up to the liners, while the Injection point is used by the different front-end concepts to locate their injection streams.

The liners reference the datums and entities as shown in Figure 4-4. These objects are used to define the inner and outer profiles, panel thickness, and the injection location for the cold stream

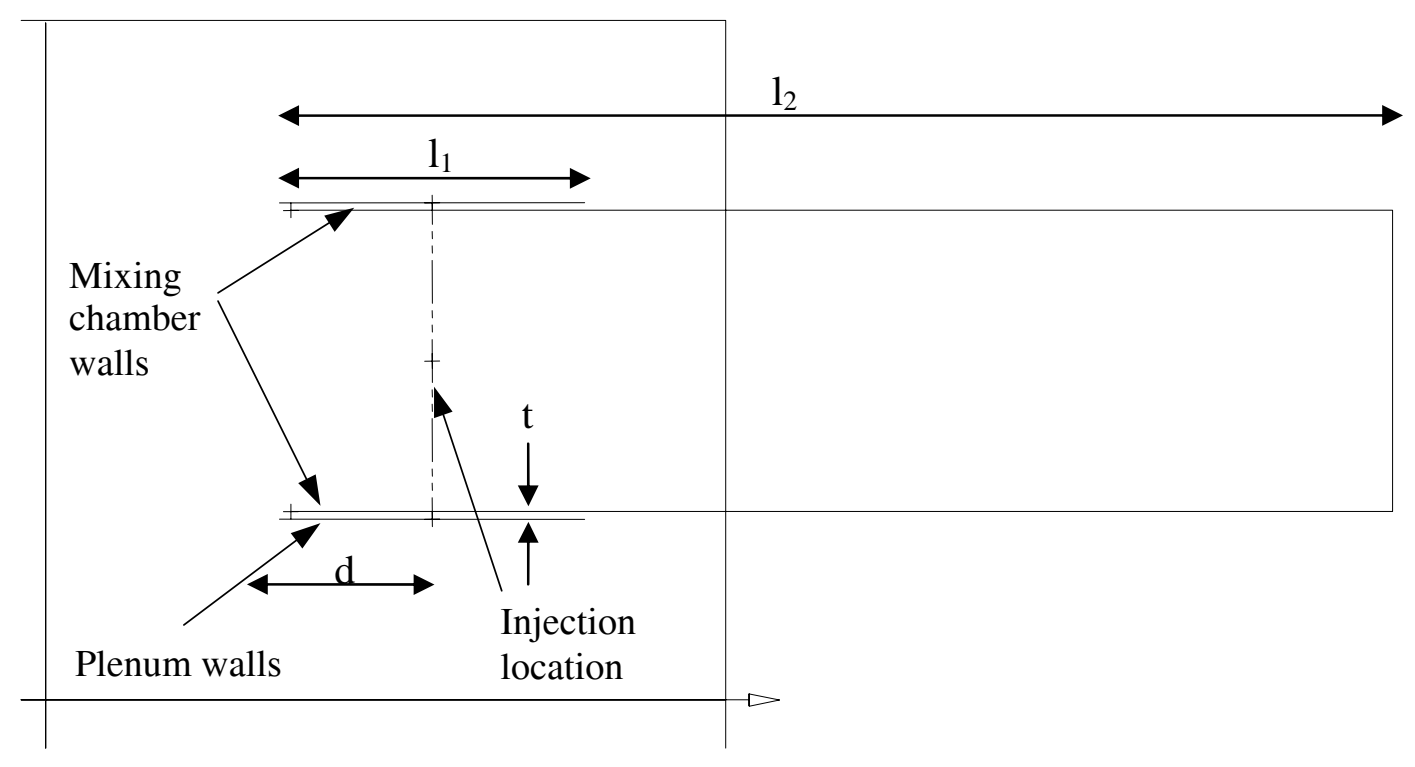

Figure 4-4 Entities referenced from the MCSS for the ID and OD liner concepts

The dashed line defines the distance $d$ downstream of the inlet, where the second stream is introduced. The shorter lines, on the top and bottom, represent the length $l_{1}$ of the outer and inner plenums. They also define the liner thickness t. The mixing chamber wall lines define the length $l_{2}$ of the mixing chamber. The points on the longer lines define where the front-end components will mate up with the liners. After the MCSS is defined, the next step is to model the components. 


\subsubsection{Component Modeling}

Each component was modeled by first adding the MCSS. After that by a technique called waving, the datums, curves, and points in the MCSS were added to the component. In Unigraphics, the concept of waving geometry allows the referencing of objects from one part to another. Standard dimensioning practices generally forbid chaining dimensions in this manner. But, by referencing these objects, any changes to the MCSS will propagate through all of the components.

For the front-end, three different concepts were selected. The first concept has an array of four rectangular openings. The second has a cylinder with a flow separator in the middle. The third has a set of four cylindrical tubes. These three different front-end concepts are displayed in Figure 4-5.
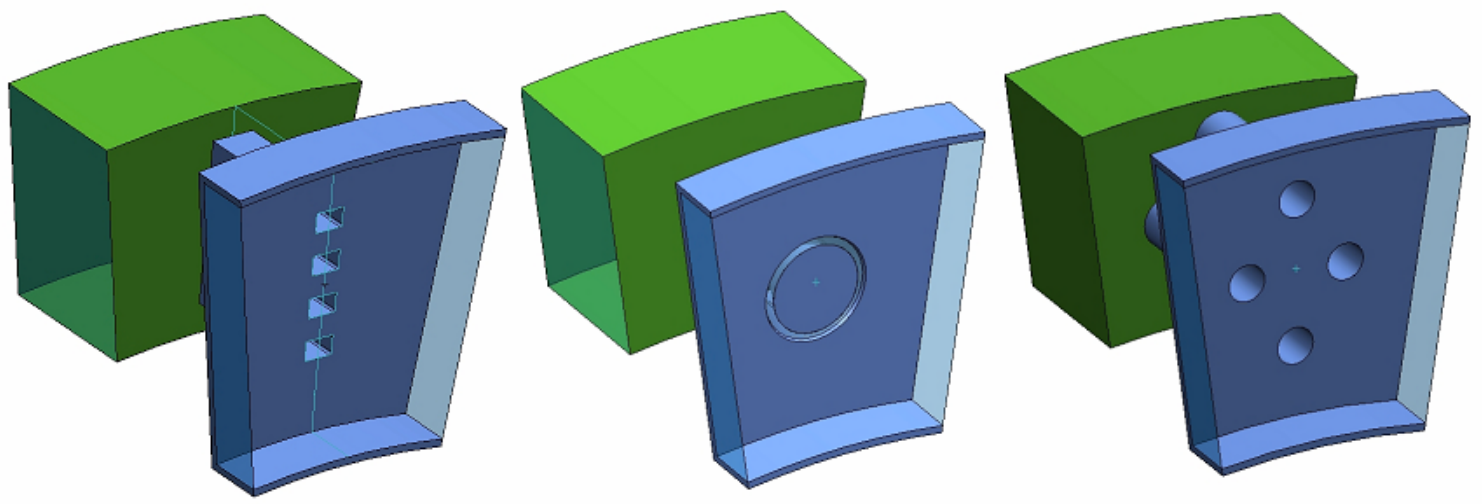

Figure 4-5 Front-end concepts

For the different liner concepts, three orifice shapes were selected. The shapes of the orifices used to inject the second stream are circular, elliptical, and triangular. These orifice concepts are shown in Figure 4-6 

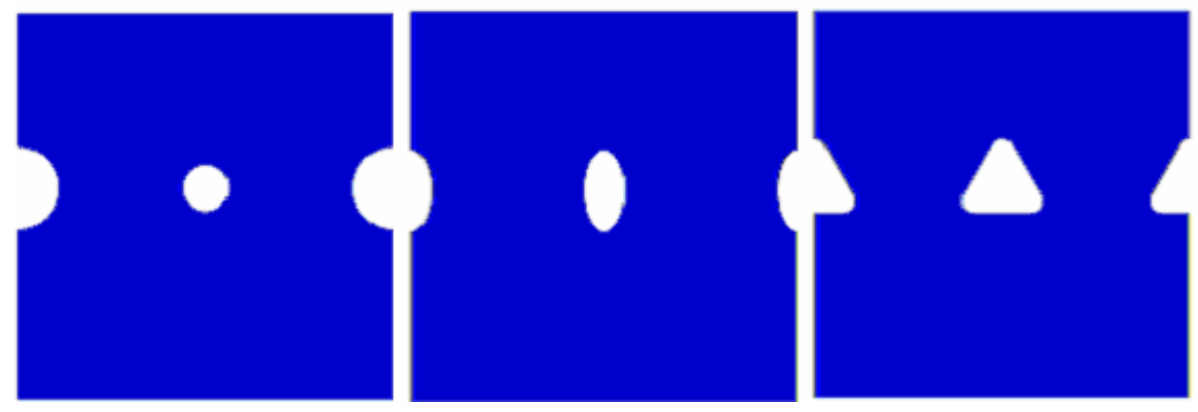

Figure 4-6 Liner orifice concepts

When constructing these concepts, certain modeling techniques were applied to ensure a valid model. First, the liner was revolved beyond the actual sector width. Second, the holes were added with the centroid of the area matching up with the second stream injection location. Each orifice was created separately to ensure the face IDs would not change. Third, all faces and curves needed for the mesh generation were tagged. Fourth, the liner was trimmed to the appropriate sector size. The reason for this is sometimes the holes will be on the periodic boundaries, which require one complete hole and two half holes. Other times the holes might not be on the periodic boundaries leaving only two complete holes. This can be seen in Figure 4-7.

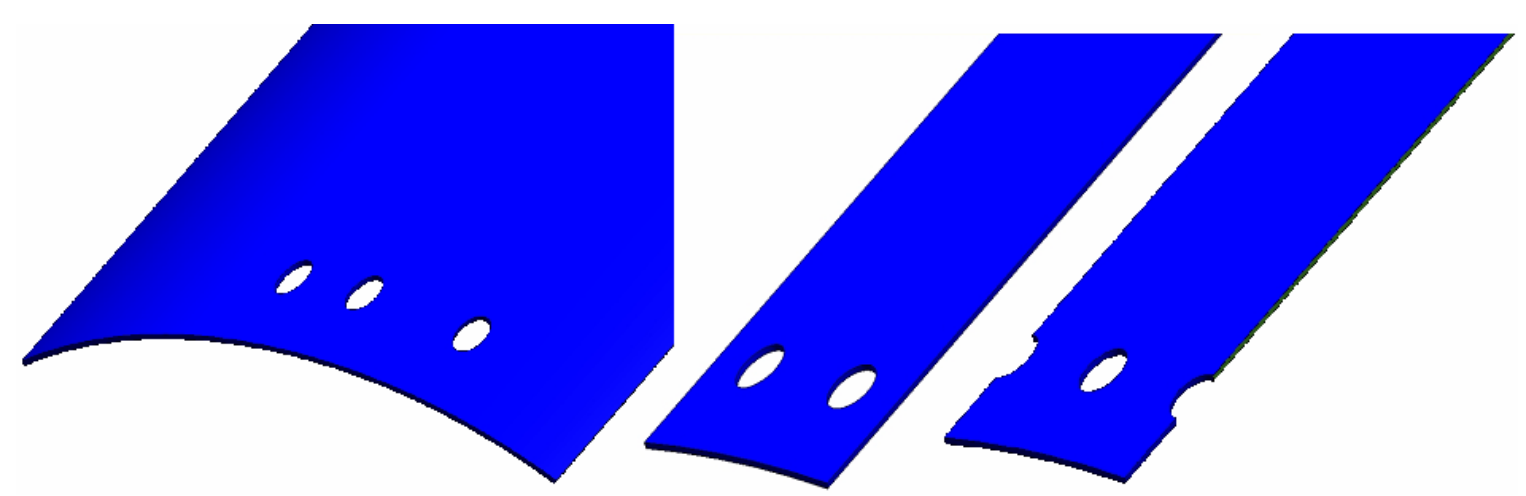

Figure 4-7 Before and after the model was trimmed 
With the concepts modeled, the next step is to match the flow area for each component concept to ensure a fair comparison. To calculate the flow area $\left(\mathrm{AC}_{\mathrm{d}}\right)$ needed for the desired pressure drop, the following equation was used.

$$
A C_{d}=\frac{\dot{m}}{\sqrt{\frac{2 \Delta p}{\rho}}}
$$

A is the geometric area of the orifice, $C_{d}$ is the discharge coefficient, $\dot{m}$ is the mass flow rate, $\rho$ is the density of the fluid, and $\Delta \mathrm{p}$ is the difference between the total pressure upstream and down stream static pressure. The $C_{d}$ parameter was used to properly size the geometric area (A) for each component. The parameters shown in List 0-1 were used to adjust the geometric area for the elliptical orifice to match the correct flow area.

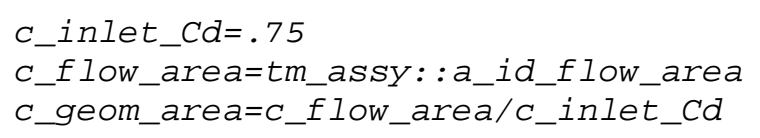

\section{List 0-1 Parameters used to calculate the necessary geometric area}

In the pseudo code $\mathrm{Cd}$ is $\mathrm{C}_{\mathrm{d}}$. The user sets c_inlet_Cd. Next the expression c_flow_area is passed from the assembly to the components, and then c_geom_area is found by dividing the $\mathrm{AC}_{\mathrm{d}}$ by $\mathrm{C}_{\mathrm{d}}$. After the geometric area was determined, the geometric area is updated.

For round holes with sharp edges and a length over diameter (L/d) around 0.2 the $C_{d}$ is approximately 0.7. For the oval and triangular holes the $C_{d}$ is unknown and must be determined. To do this a $C_{d}$ was guessed for the oval and triangle holes. A guess of 0.75 and 0.6 were selected respectively. To find the actual $C_{d}$ for these holes, CFD simulations 
were performed. The $C_{d}$ for the holes was calculated by using Equation 4-2 at the measured pressure drop from the CFD simulations. The values calculated for $C_{d}$ for the different concepts are shown in Appendix A.

After front-end and liner concepts were modeled, they were assigned object attributes to resolve the geometry for the mesh generation step. The required faces, curves, and points were tagged using ICEM's UG Mesher interface tool. For example, the faces tagged for front-end concept \#3 are shown in Figure 4-8.
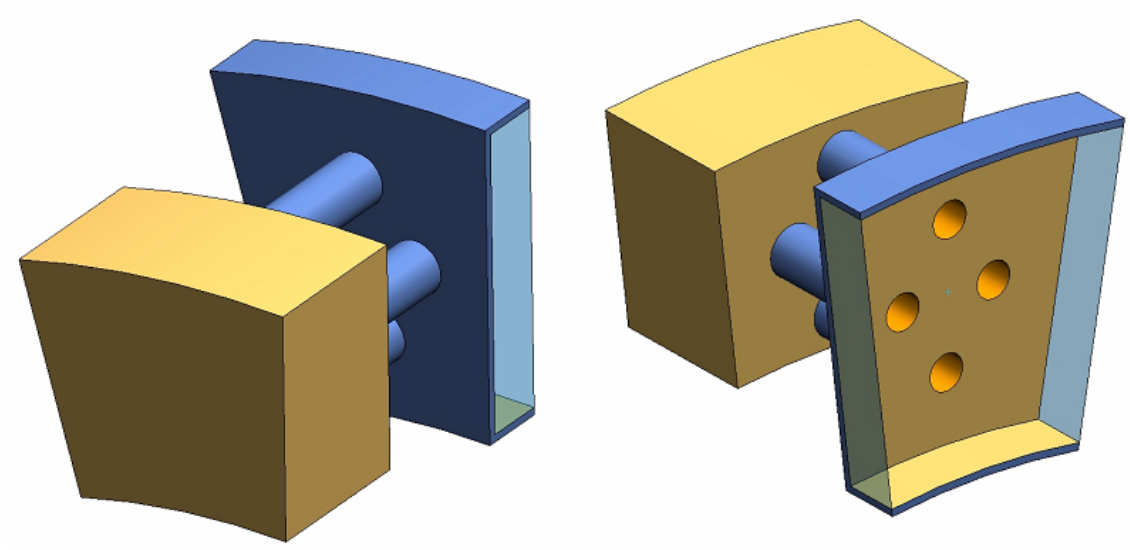

Figure 4-8 Surfaces tagged for mesh generation.

The gray face on the right was tagged as the inlet family. The rest of the lighter faces were tagged and assigned to their respective families. The edges of the selected faces were also tagged. A screen grab from ICEM shows the families, faces, and curves tagged for the mesh generation step in Figure 4-9. 


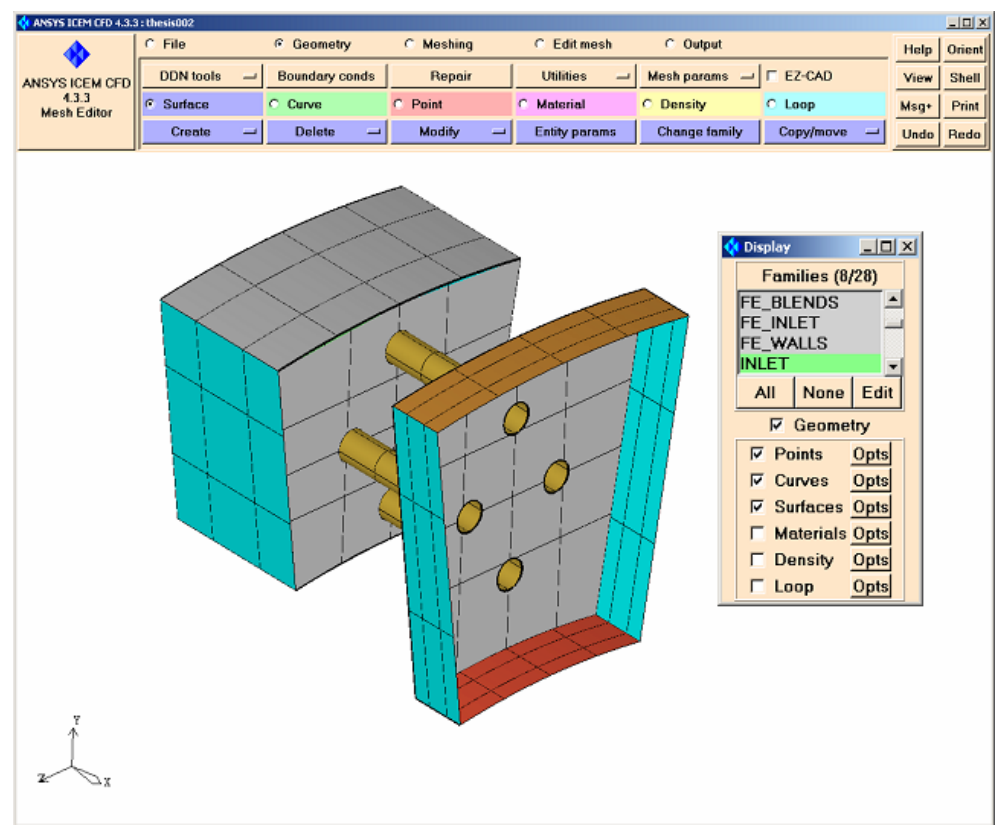

Figure 4-9 Family, faces, and curves that were tagged for front-end concept \#3

The other front-end concepts were tagged in a similar manner. For the liners, just the surfaces were tagged and assigned families, since they will be checked with the geometry processor.

\subsubsection{Assembly Modeling}

Adding the MCSS first, and then the components, the assembly model was constructed. To define which components are present for a given configuration, “controlled by expression” parameters were used to toggle them on and off. Next, an air solid was created using entities from the MCSS and revolving them. The air solid without the front-end or liners subtracted can be seen in Figure 4-10. 


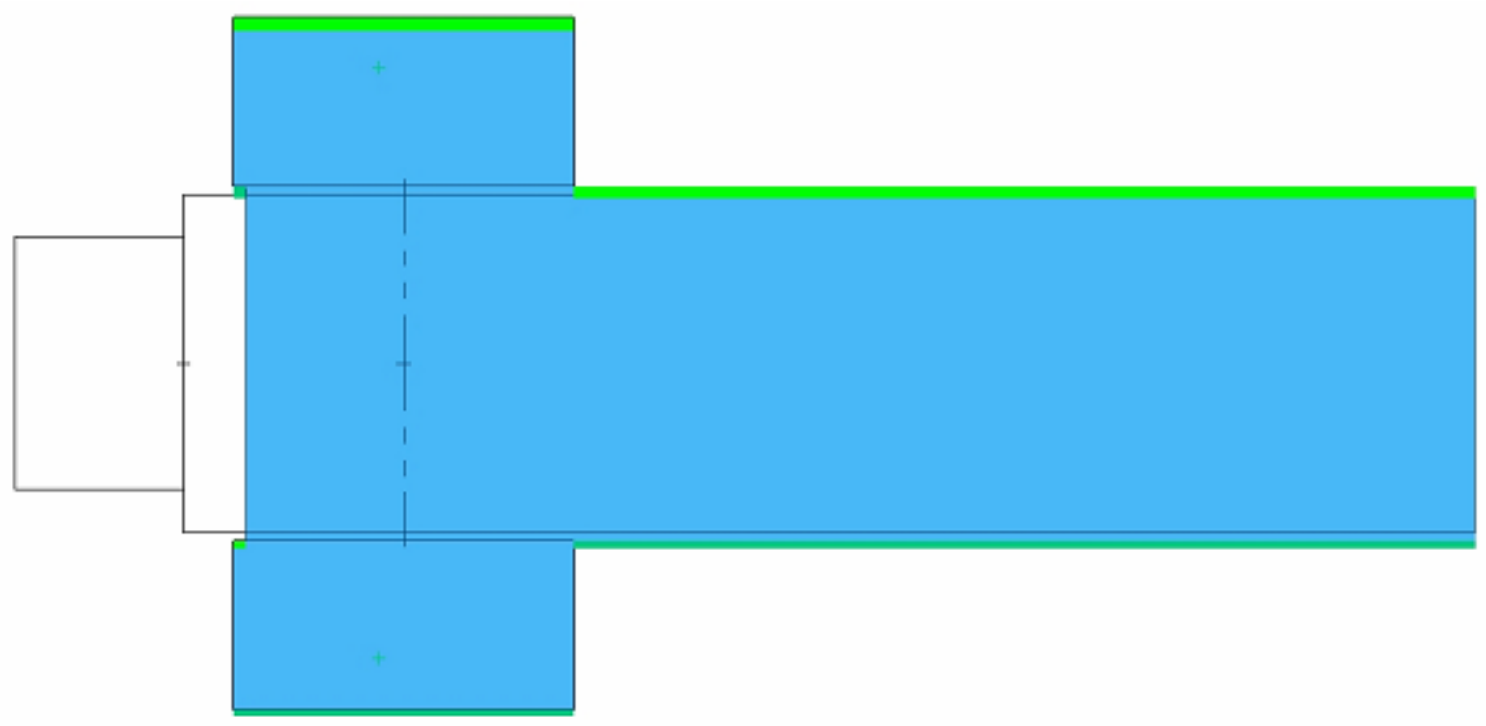

Figure 4-10 Initial air solid

As seen in the figure above, there is no solid portion for the different front-end concepts to be subtracted from, due to the different topologies for each front-end. The different front-end concepts have added to them two sheet bodies to provide periodic and inlet(s) surfaces in order to close off the air solid. These added sheet bodies are shown in Figure $4-11$

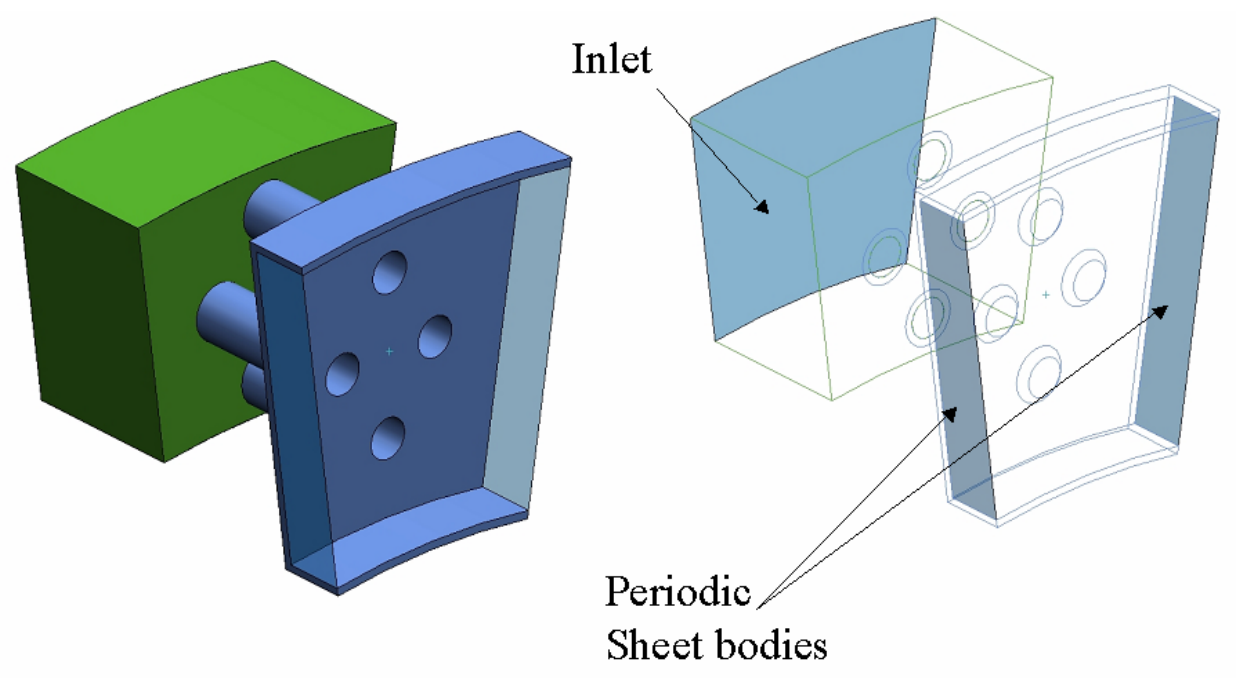

Figure 4-11 Sheet bodies used to close the air solid 
Next, the OD and ID liners were subtracted from the air solid. Figure 4-12 shows the resulting air solid.

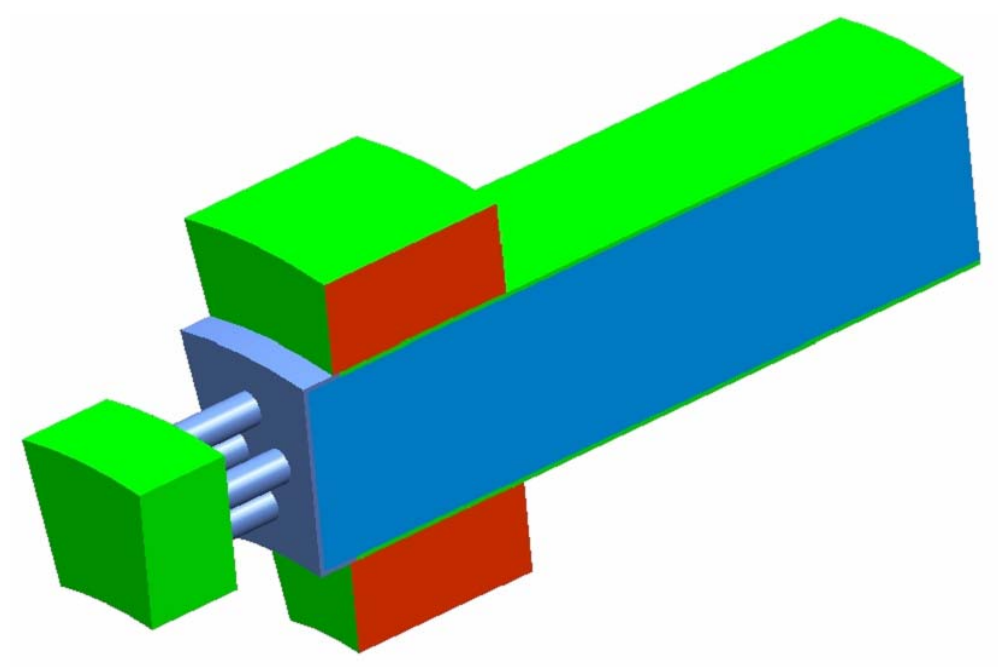

Figure 4-12 Air solid model

When the components were added to the assembly and removed using “controlled by expression”, a few problems arose. First, a linked body was used to subtract the component from the air solid. When one component is toggled off and another on, the linked body of the inactive component remained, causing two of the same component to be subtracted from the air-solid. For example, when the triangular orifice was unsuppressed, the oval orifice remained. This is shown in Figure 4-13. 


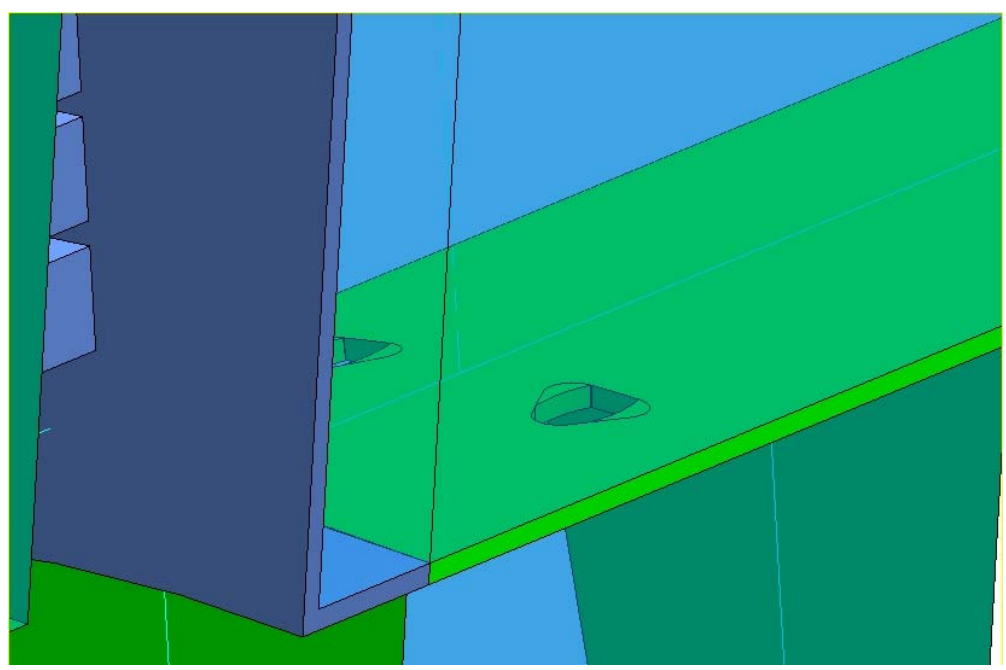

Figure 4-13 Assembly issues when a component is toggle off and another one is toggled on

To resolve this issue, a feature set was created for each linked body and subtraction feature. Then, an expression was created, that would suppress the feature set when the component was suppressed. When this was done the assembly model updated correctly for all configurations.

With the air solid completed, the assembly was changed to a state which contained all of the potential periodic faces. This was done, by moving the injection holes on the ID and OD liners off of the periodic faces. The three periodic faces which resulted, are shown in Figure 4-14. These faces are the dark faces on the side of the OD and ID plenums and the lighter face on the mixing chamber. 


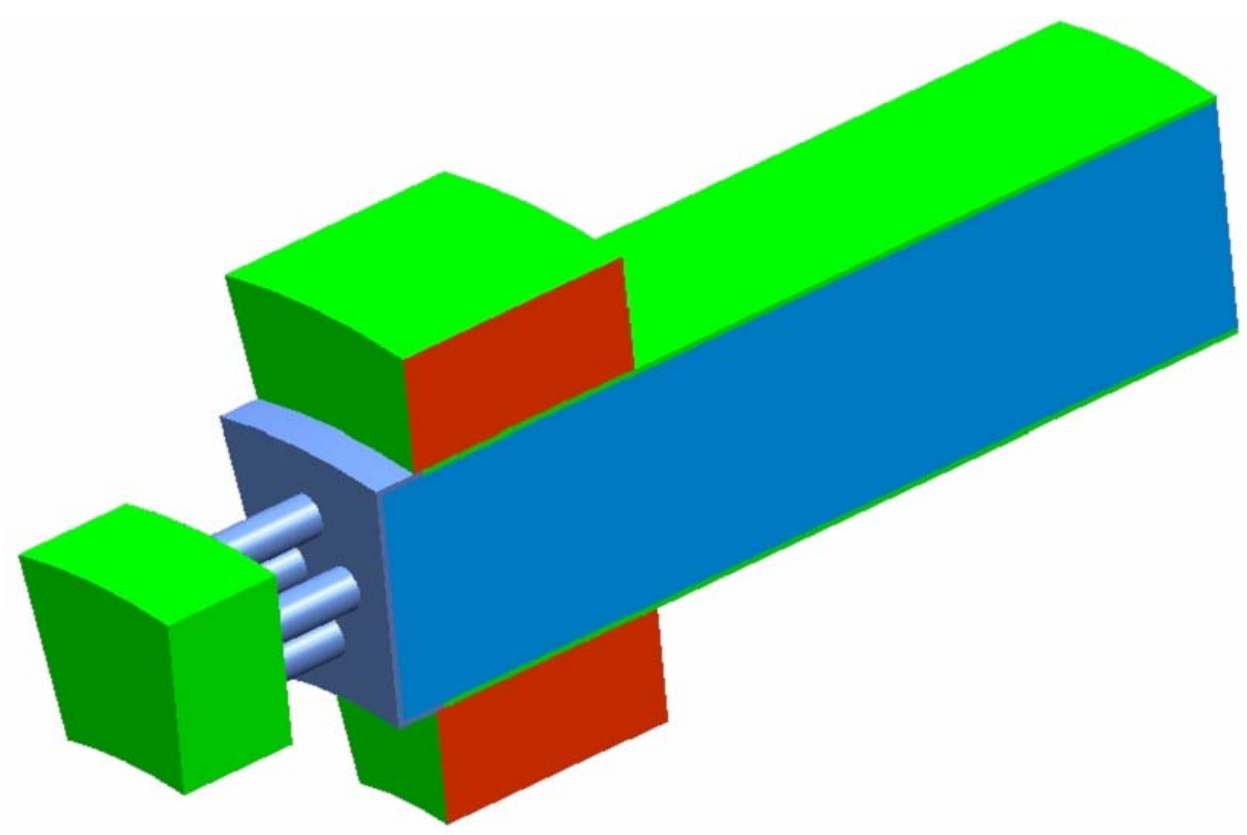

Figure 4-14 Air solid with 3 periodic faces

In this configuration, the three faces were tagged as periodic faces. This was done to ensure that any new configuration of this model would have the periodic faces tagged. The model was then saved in this state as the master model. The model at this point was ready for the geometric processor.

\subsection{Geometry Processor}

The geometry processor is used to prepare the CAD model to be gridded. The geometric processor reads in the input file, opens up the CAD model, imports the expression file, updates the model, and check for the attribute names defined by the user, which may contain topological changes. The topological changes include the number of edges and end points that are needed to resolve the geometry. The input file used in this thesis is shown in List 4-2. 


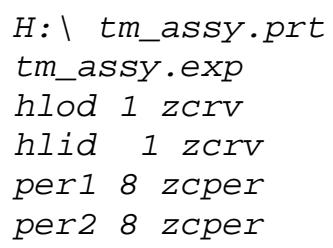

\section{List 4-2 Input file for geometric processor}

The first line tells the geometric processor which CAD model to open. The second line indicated the name of the expression file to use to update the model. The rest of the lines define the families to check, mesh size to assign to the curves, and the name of the families to assign to the curves. The commands used to open the part and update it with the specified expression file that is specified in the input file is shown in Code Frag 4-1.

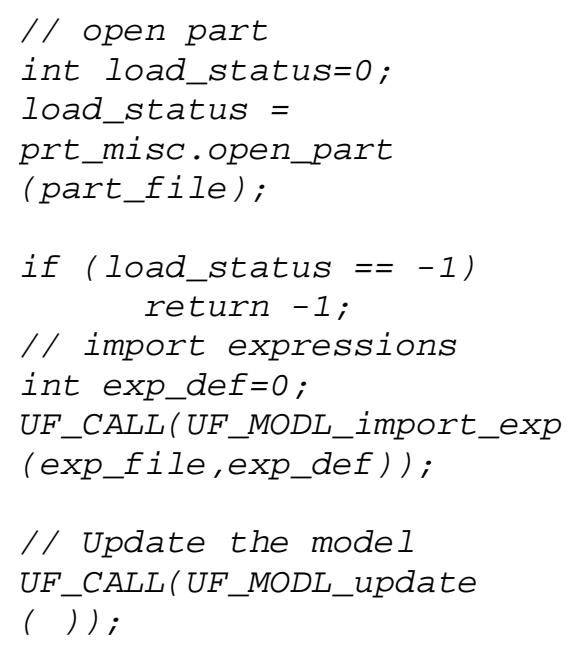

Code Frag 4-1 Code used to open the CAD model, read in the expression file and update the model

When the CAD model is updated, the new edges and points are tagged. This tagging was done using Code Frag 4-2. 


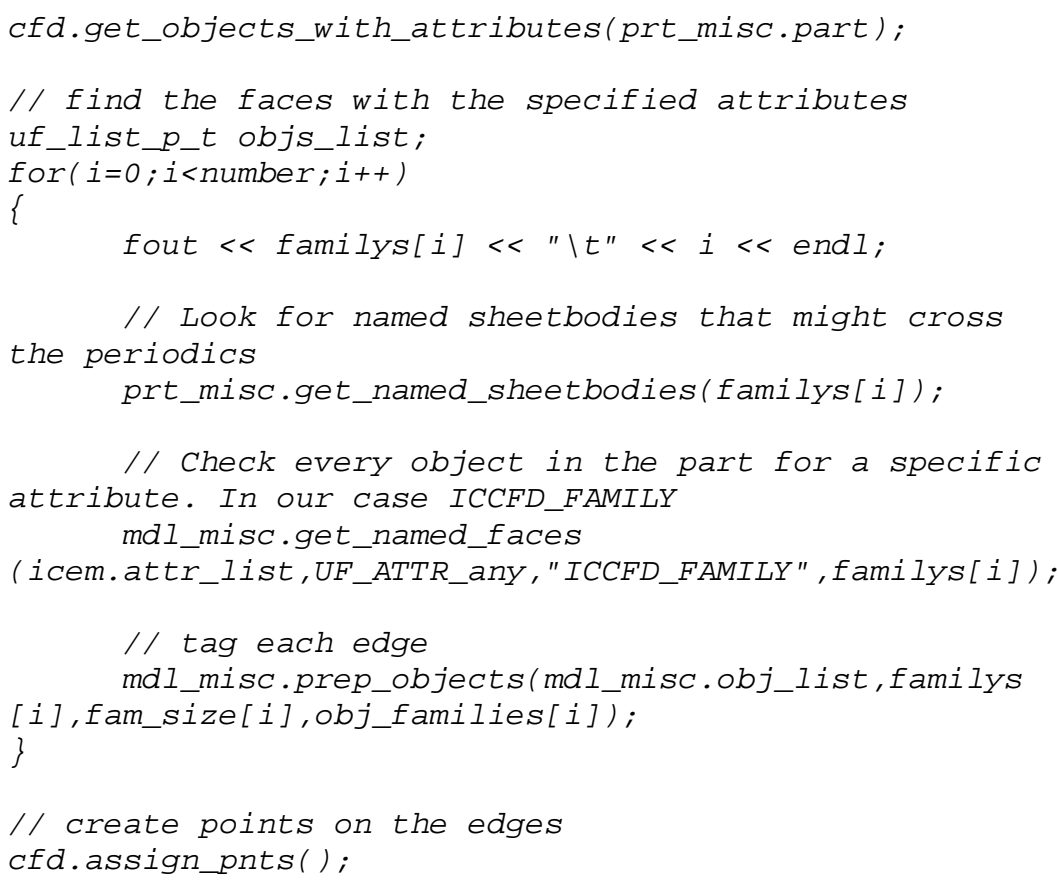

\section{Code Frag 4-2 Find the specified family edges and add points and tag them}

When the model has been processed, it was saved into the current working directory with prefix "mod_" added to the part name. Some images of ICEM input files are shown in Figure 4-15. They indicate the input files generated with and without the geometry processor. As seen in the before and after images below, curves and points were added on the outer diameter to the injection holes, mixing chamber, and plenum 
Before
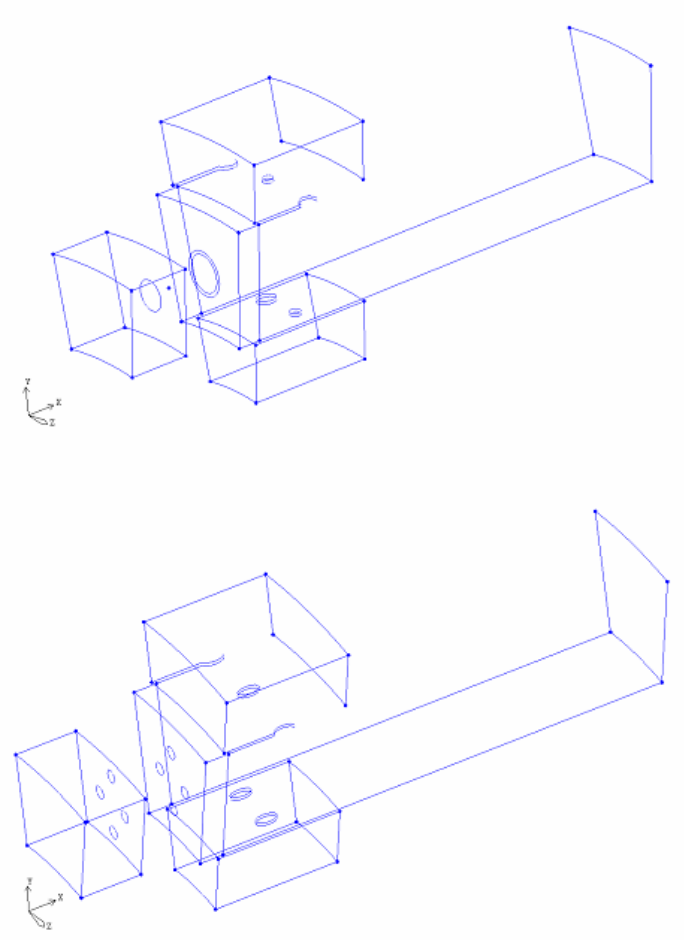
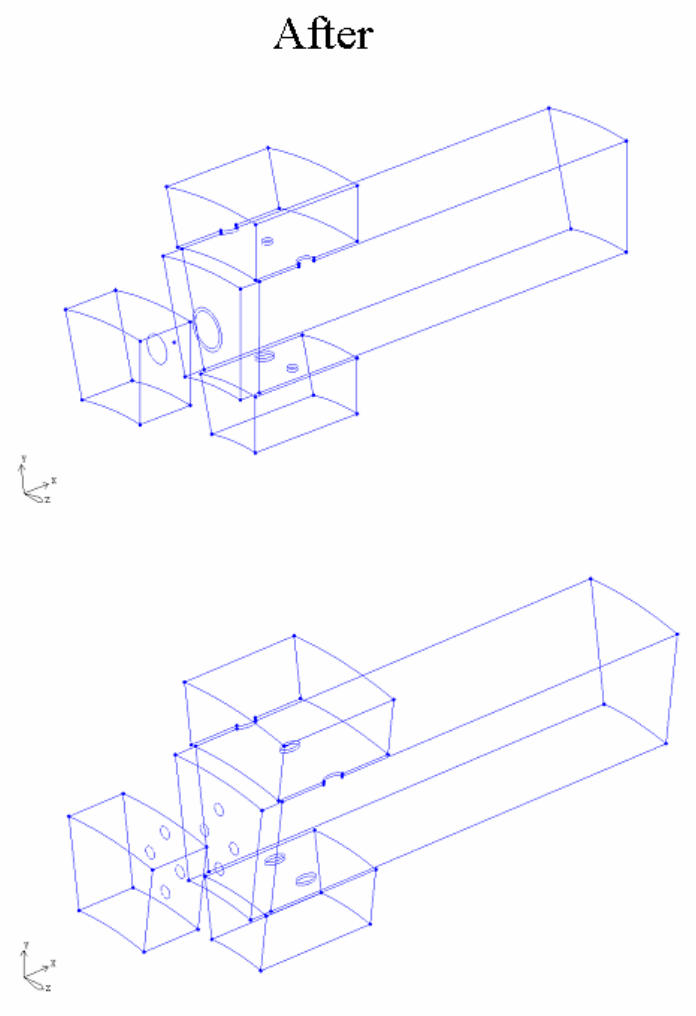

Figure 4-15 With and without running the geometry processor

\subsection{Grid Generation}

To automate the grid generation process a Tcl script was created. This script was broken up into modules for reusability. This allows for new modules to be added by using the line "source ..." as shown in Code Frag 4-3 


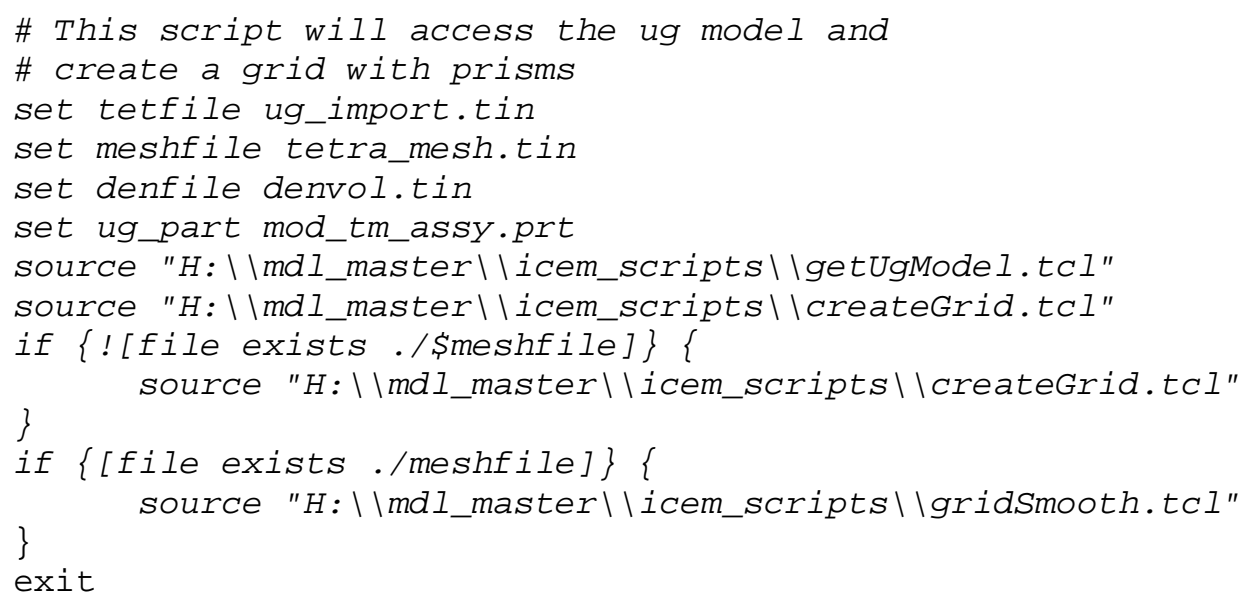

\section{Code Frag 4-3 Script created to automate the grid generation process}

The set command is used to set variables used in all of the modules. After the variables were set, then the UG model was imported using the getUGModel.tcl module.

Then the script calls the createGrid.tcl, which creates the grid input file called ug_import.tin. Next, a file containing the density volumes was merged with the input file and saved. These steps are shown in Code Frag 4-4

\section{Code Frag 4-4 Code to import geometry from Unigraphics and add density volumes}

After the tetin file was created, the grid was created and smoothed using the following command: 
ic_run_tetra./\$tetfile./tetra_mesh.uns run_cutter 1 run_smoother 0 smoother_n_iter $\odot$ smoother_aspect 0.4 n_processors 2 log tetra_cmd@.log

This command reads in the ug_import.tin file, creates a mesh called tetra_mesh.uns, turns the smoother operations off, and specifies the uses of two processors. The tetra_mesh.uns file was then smoothed using the following command

ic_smooth_elements upto 0.3 metric Quality iterations 10 prism_warp_weight 0.5 no_collapse 1 smooth TETRA_4 smooth TRI_3

This selects any tri_3 of tetra_4 elements under a quality of .3 to undergo up to 10 smoothing iterations until the element quality exceeds .3.

When the model is smoothed it will be saved for visual inspection by using the following command

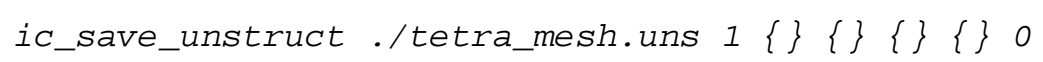

Visual inspections were needed to ensure that the geometry was imported correctly and the resulting grid was valid. After this the valid grids were export to a PATRAN neutral file format for analysis.

\subsection{CFD Analysis}

The analysis phase consists of specifying the boundary conditions, preprocessing the grid generated in ICEM, analyzing the model, and postprocessing the solution to visualize the results.

\subsubsection{Preprocessing}

For the cases in this study, preprocessing is needed to read in the grid from ICEM, which converts it to a model file that can be used by the solver. The model file consists of the grid, with the boundary condition properly assigned to the necessary nodes. After the 
model file is created, it is broken up for parallel processing. At this point the model is ready to have boundary conditions specified.

\subsubsection{Boundary Conditions}

For the two stream mixing problem, the mass flows of the two streams were specified. The hot stream flow rate was set to $0.033 \mathrm{lbm} \backslash \mathrm{s}$ at $500{ }^{\circ} \mathrm{F}$. The cold stream flow rate was set at $0.066 \mathrm{lbm} / \mathrm{s}$ and $70{ }^{\circ} \mathrm{F}$. The desired pressure drop across the liners was set to be 3\%. The table used to specify the boundary conditions (BC's) can be seen in Appendix A. To determine the flow velocity into the plenums, equation 4-2 was used:

$$
V=\frac{\dot{m}}{\rho A}
$$

where $\mathrm{V}$ is the velocity, $\mathrm{m}$ dot is the mass flow rate, $\rho$ is the density of the fluid, and $\mathrm{A}$ is the flow area. Using this equation the mass flow rate for the first and second streams was calculated. The velocities calculated for the different plenums can be seen in Figure 4-16

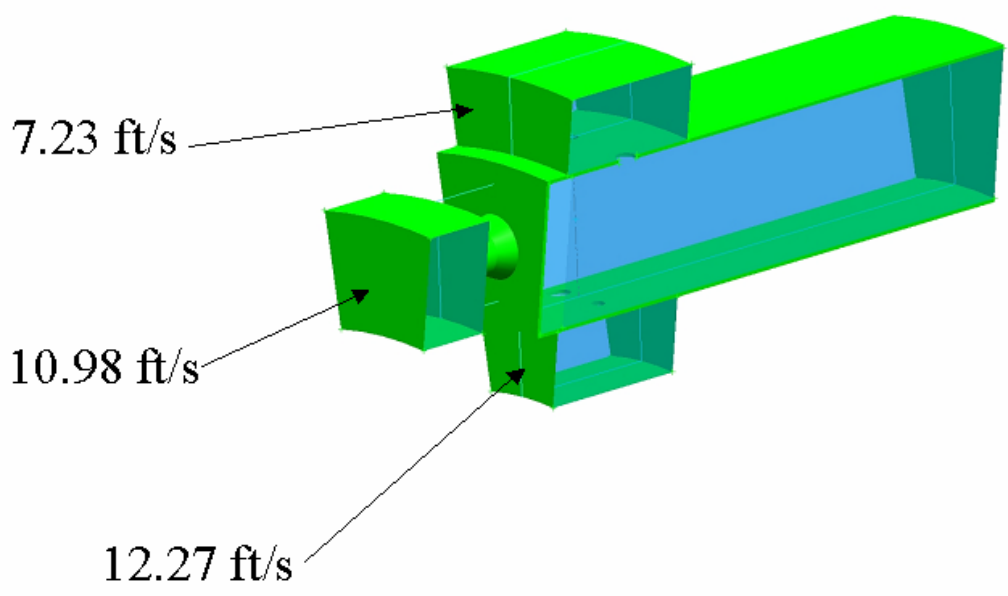

Figure 4-16 Flow velocities into the plenums.

With the boundary conditions specified the next step is to solve the model. 


\subsubsection{Analyzing the Model}

To arrive at a solution, the solver iterated until the parameters converged to their final values. It was determined that it required around 2000 iterations for the solution to converge. When converged, the solution was checked to verify that the flow field had converged. Next, each case was post-processed to view the results.

\subsubsection{Postprocess the Results}

The postprocessing of each case was done using an Ensight command file. The command file was a macro file that was generated by interactively recording the steps used to postprocess the case. The file was then edited to repeat certain commands at different locations, calculate variables, and generate images of the flow field for the desired variables. For each case, the script first opens the model and reads in the results. This was done using Code Frag 4-5.

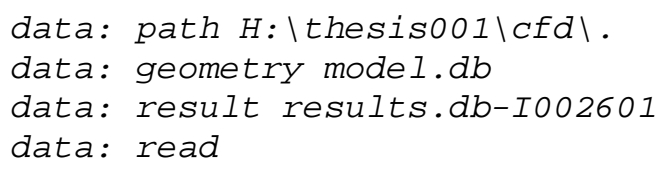

\section{Code Frag 4-5 Ensight commands to read in the model and results}

When the model is read in, different screen grabs are taken to display the model and the grid. Next, a pressure map down the centerline of the model was created. This provided a visual picture of the pressure field as a sanity check. Next, the script took point measurements in the flow field to find the pressures that were used to calculate the pressure drop. When the pressure portion of the script was complete, the script generated temperature slices of the flow field at different locations to visualize the mixing of the 
flow field. At each slice, a maximum and a minimum temperature were recorded. Finally, the script wrote all of the calculated scalar variables into an output file.

\subsection{Design Review Tool}

Upon completion of the post processing of a case, the Design Review Tool (DRT) was employed to document each solution. It provided a standard method to review each solution. The DRT is a Python script, used to generate Hyper Text Markup Language (HTML) pages. The HTML pages document each case.

The DRT script was composed using different classes to provide reusability. To set up the DRT, certain variables were modified to indicate the location of the study directory and the prefix used for each CFD case. The lines in the DRT that were updated for this project are shown in Code Frag 4-6.

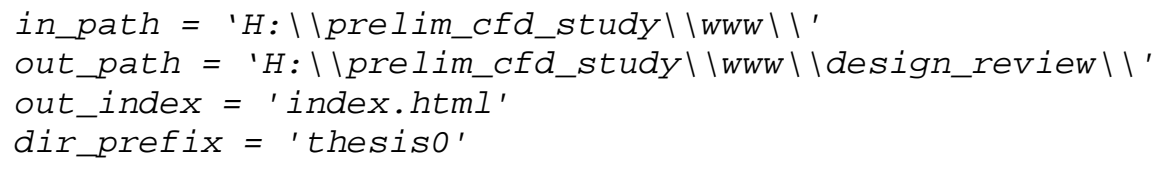

\section{Code Frag 4-6 Input needed to define the location of the files for the DRT}

The in_path specifies where the solutions were located, out_path indicates the directories where the solutions are copied to, out_index is the name used for the index page, and dir_prefix indicates the prefix of the solution directories. With these variables updated to reflect the location of the files, the Design Review is ready to be run.

To allow for code reusability, each section used on the case page was created using separate modules known as classes. The model class used to create the model section is shown in Code Frag 4-7. It takes the file model.jpg created in the Ensight script, along with the expression file used to define the UG model and displays them in a 
web page. If the user wanted to display more information or change the formatting, all that is required is to modify the model class and rerun the DRT. The other modules were set up in a similar manner.

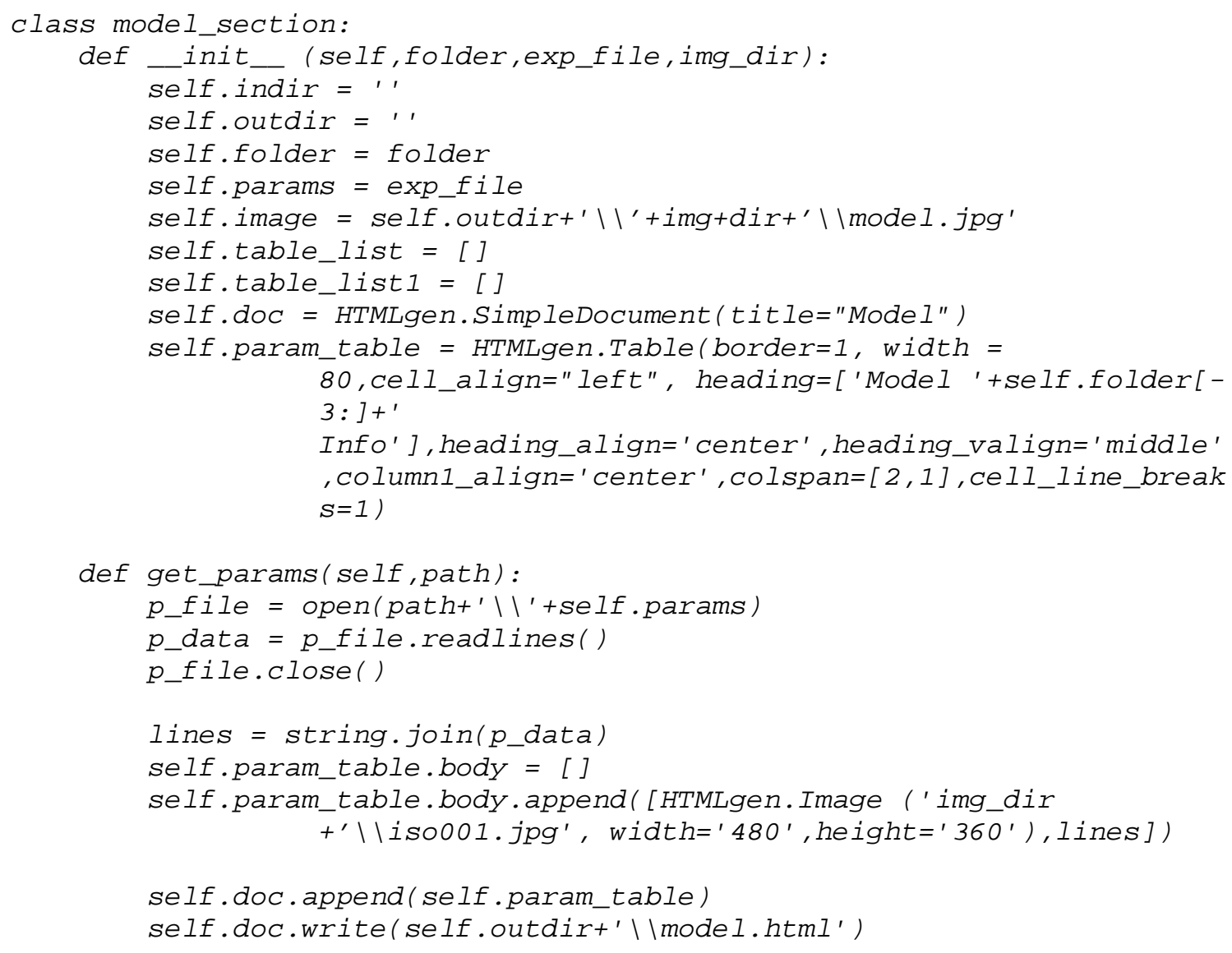

\section{Code Frag 4-7 Model class module for the DRT}

The first web page created by the DRT displays a listing of all of the cases with the predicted temperature spread at the exit plane as seen in Figure 4-17. 


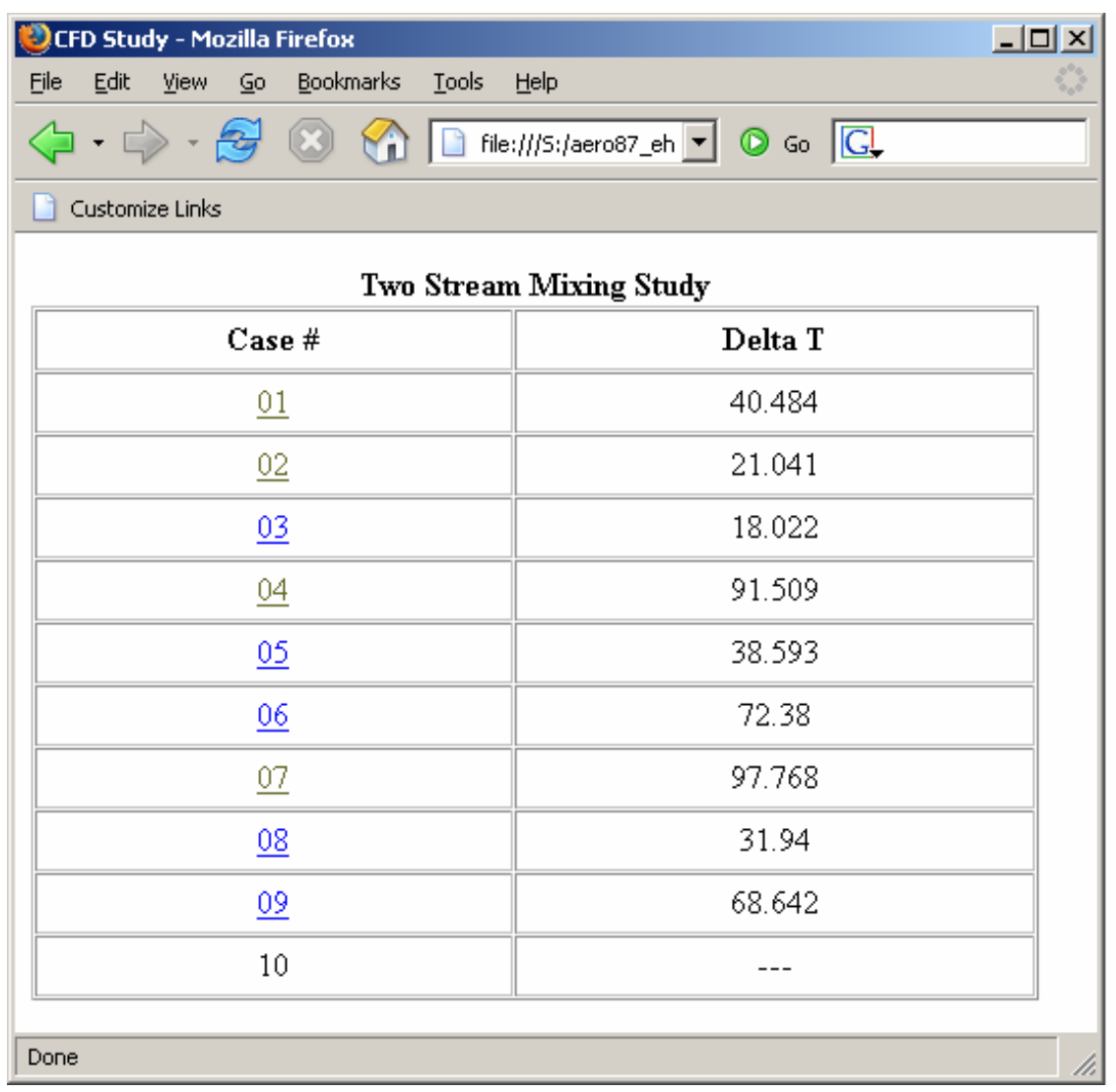

Figure 4-17 A screen grab of the preliminary CFD study page

From the "Two Stream Mixing Study” page, the design team can see which cases are completed with the objective value and which cases are in progress. The finished cases are selectable and their results can viewed by selecting a case number. The cases that are in progress display a "---” in the objective column and are not selectable. By selecting a case number for a completed case, the case page is displayed. This page provides a quick glance at the different sections of the case as seen in Figure 4-18 


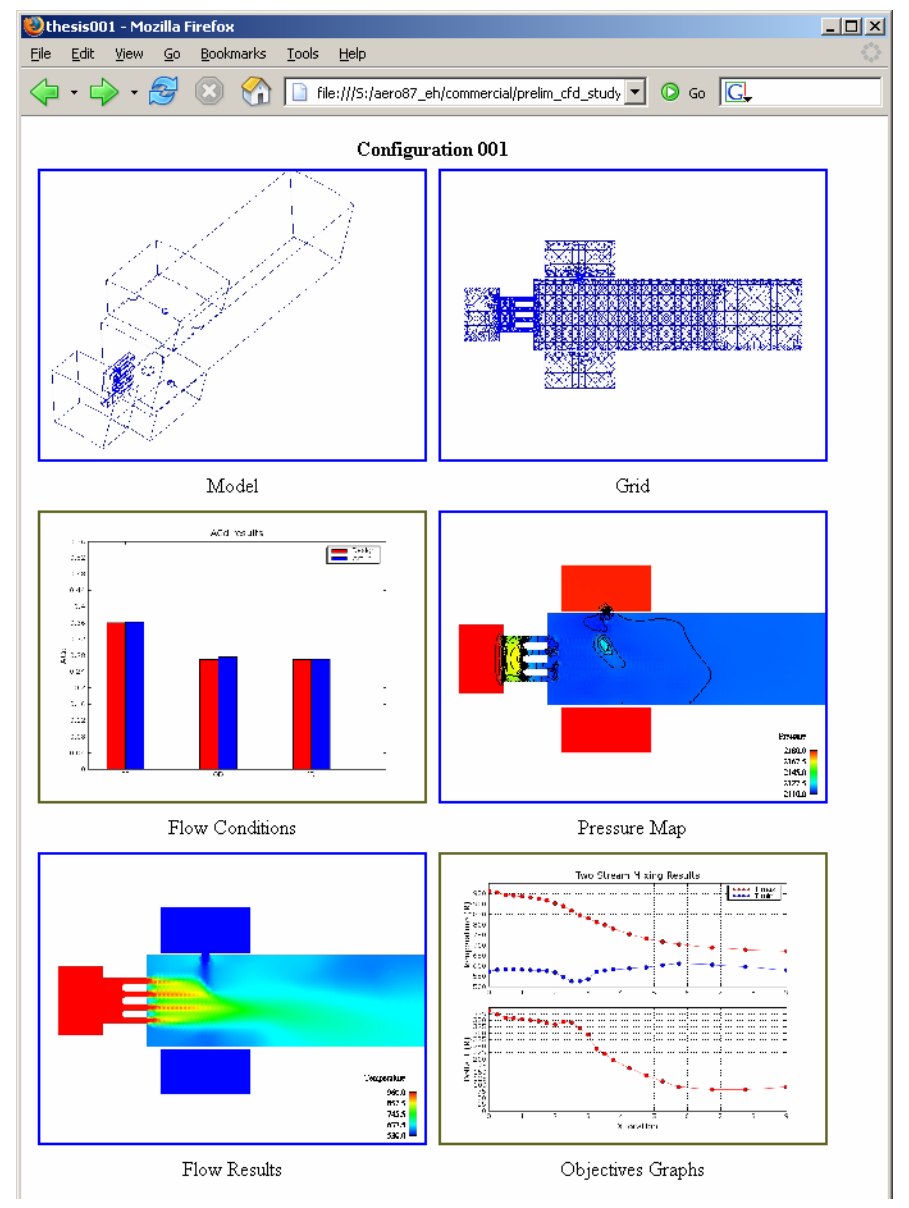

Figure 4-18 DRT: Case template

Each section can be reviewed by clicking on an image. This allows the user to review the model and solution. The model and grid section provides a review of the geometry and the grid. The model section displays a wire frame view of the model, along with the expression file used to update the CAD model. The page that is displayed when the model image is clicked is shown in Figure 4-19. The grid section shows a slice of the grid, with a table listing the families and elements that are tagged as boundary conditions. The page that displays when the grid image is clicked is shown in Figure 4-20 


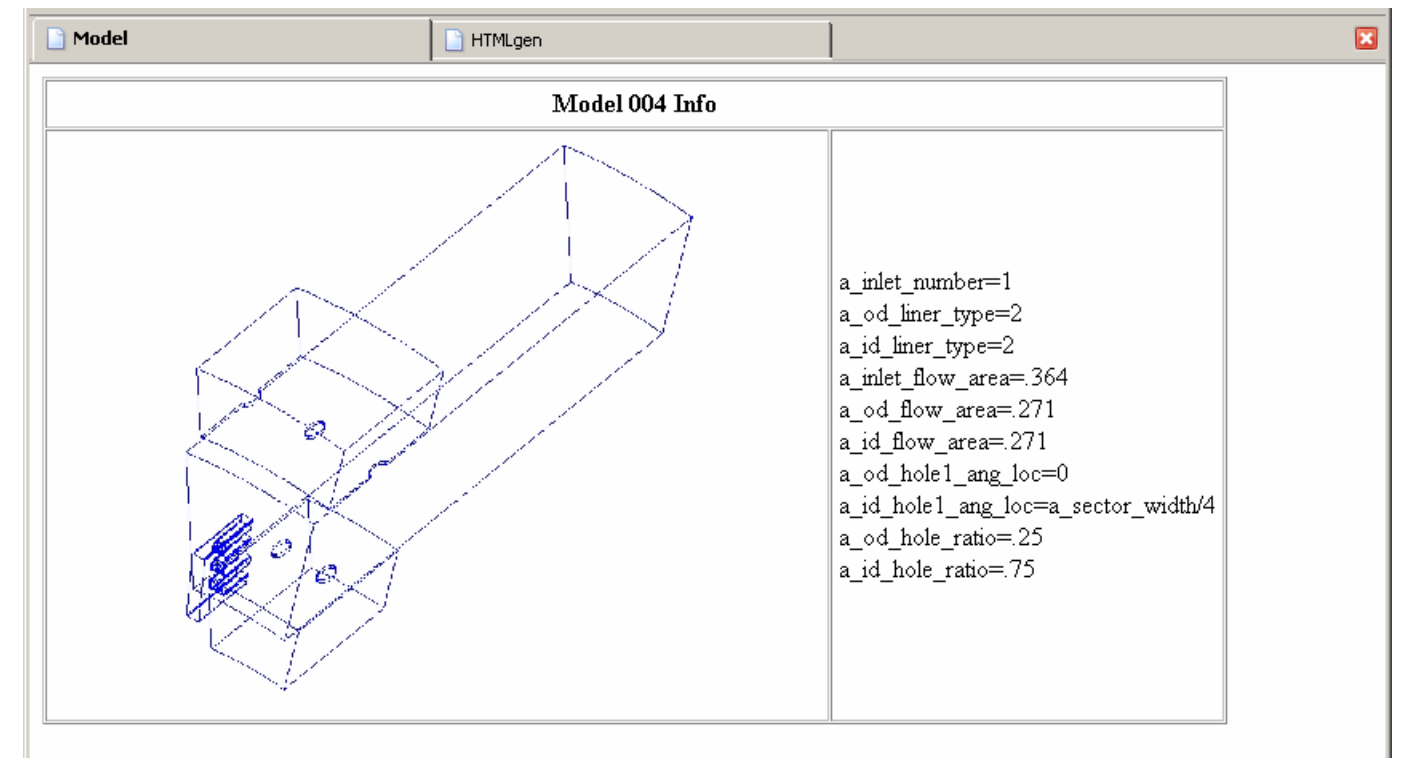

Figure 4-19 DRT: Model section

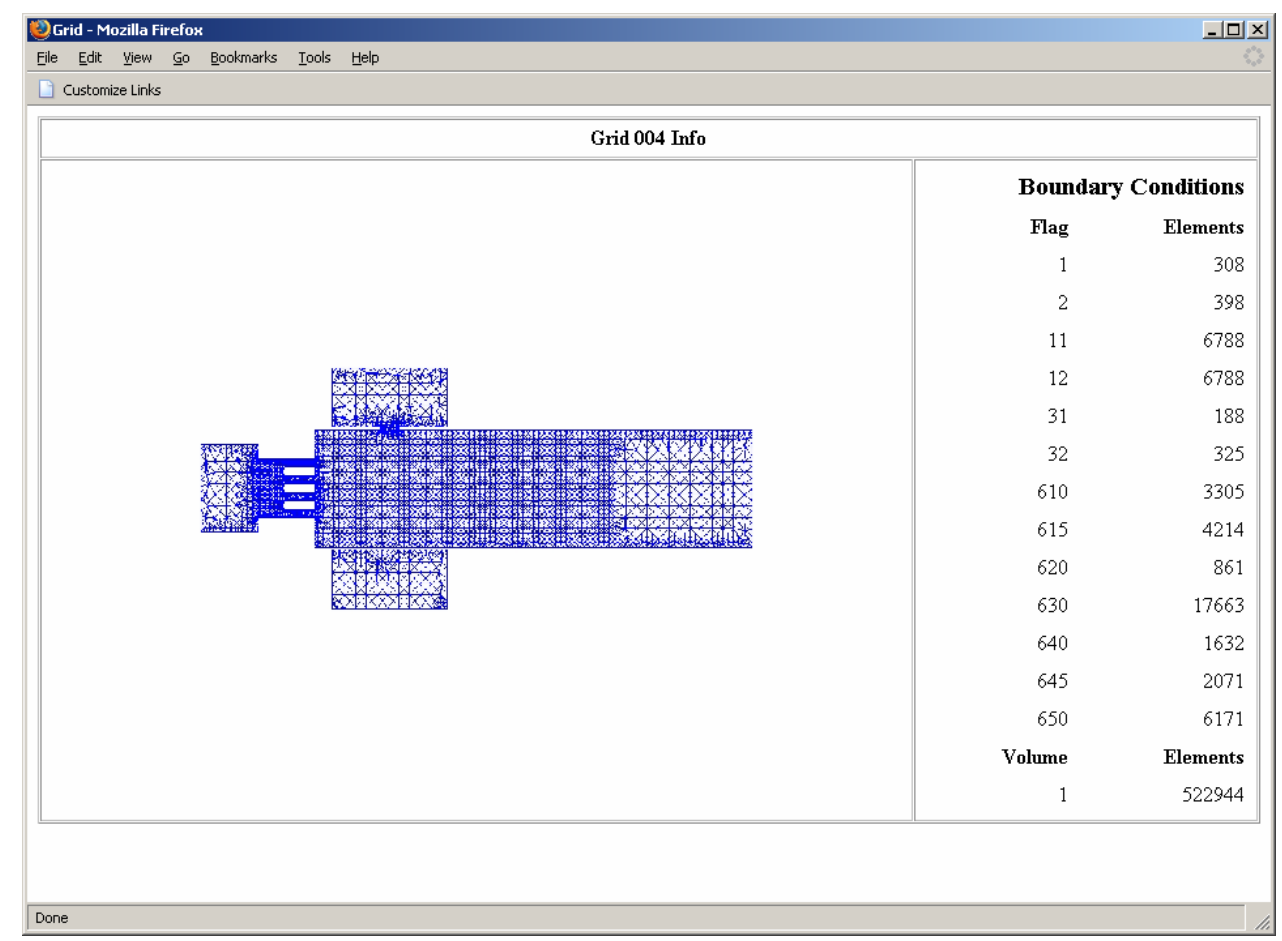

Figure 4-20 DRT: Grid section

The next two sections, flow conditions and pressure map, help verify that the

CFD solution was run properly. The flow condition page, shown in Figure 4-21, provides 
a visual check of the flow conditions. While the pressure map page, shown in Figure 4-22, provides a visual inspection of the pressure field.

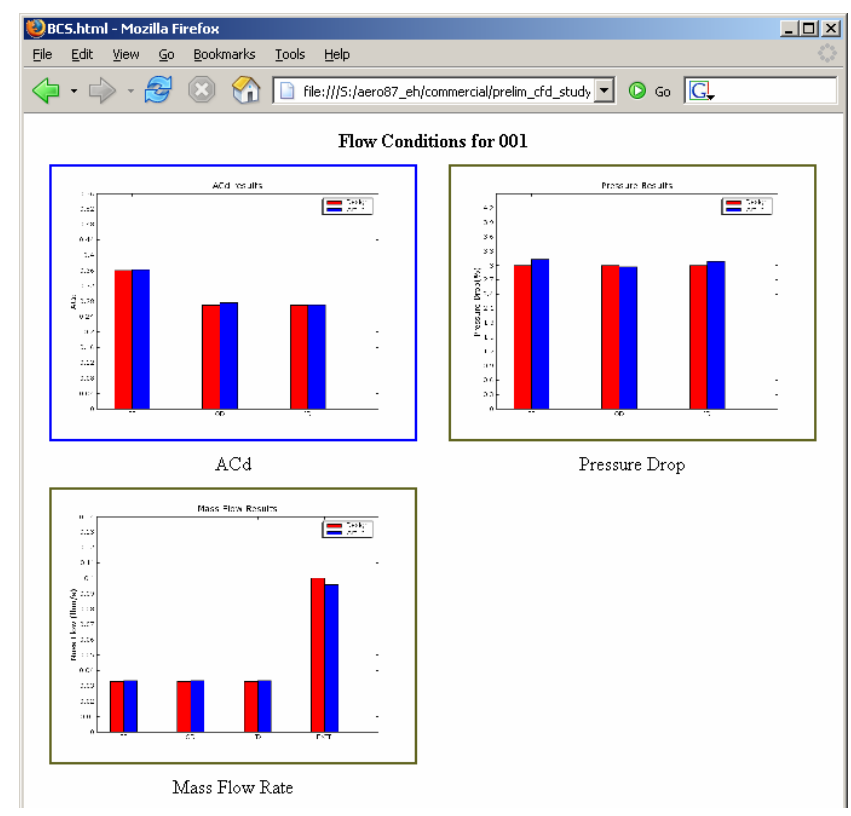

Figure 4-21 DRT: Flow conditions section

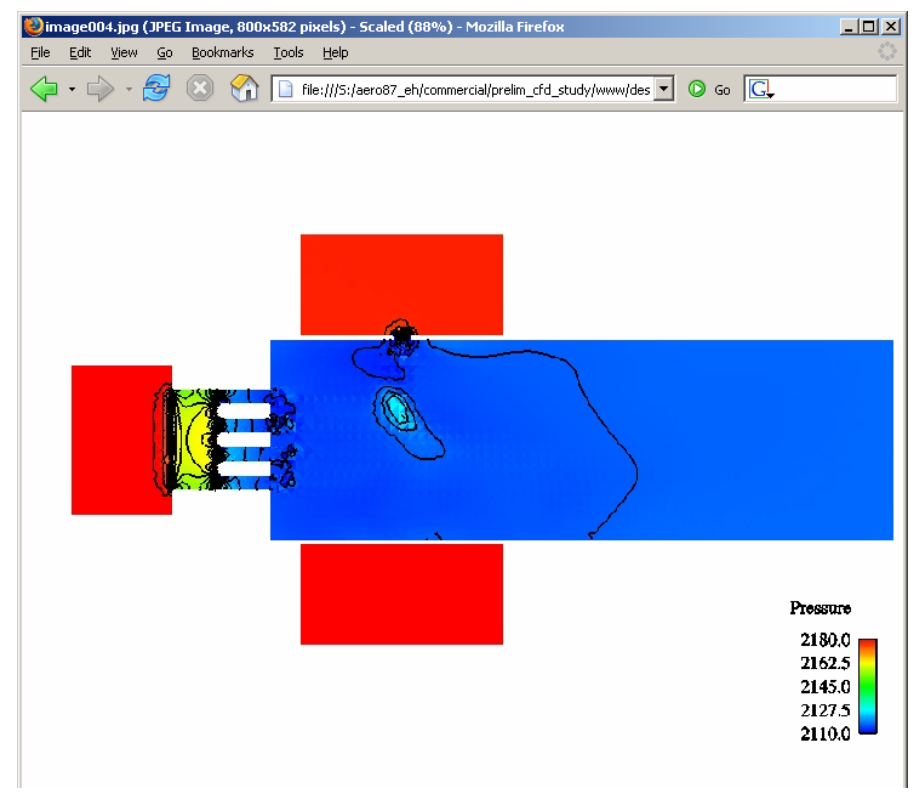

Figure 4-22 DRT: Pressure map section 
The final two sections, flow results and objectives, provide a visual representation of the results for each case. In the flow results section, different variables can be reviewed. For this thesis, only two were look at, however, if more images were created they could easily be added to this section by modifying the flow results module used by the DRT.

The page that is displayed when the flow results section is clicked on can be seen in Figure 4-23. From the flow results section page the different parameters are shown and can be reviewed by clicking on the image. When the temperature image is clicked on, all of the temperature images generated are displayed. A screen grab of the temperature page is shown in Figure 4-24. At the top center of each result page is an image that shows the axis for the different slices. The top center image for the temperature results can be seen in Figure 4-25.

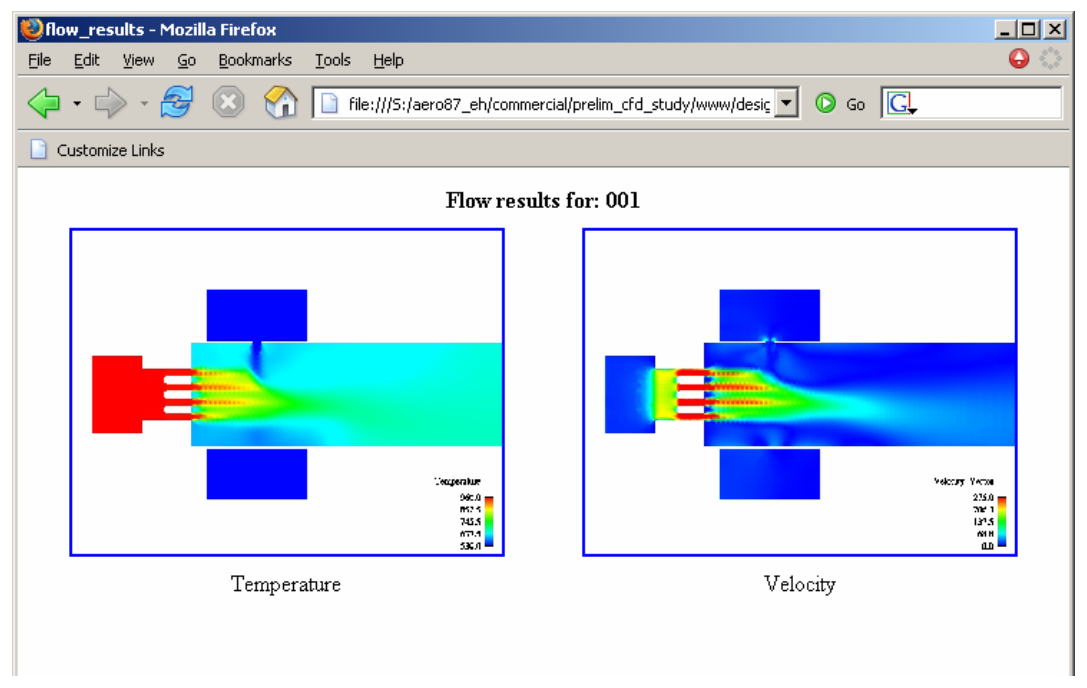

Figure 4-23 DRT: Flow results section 


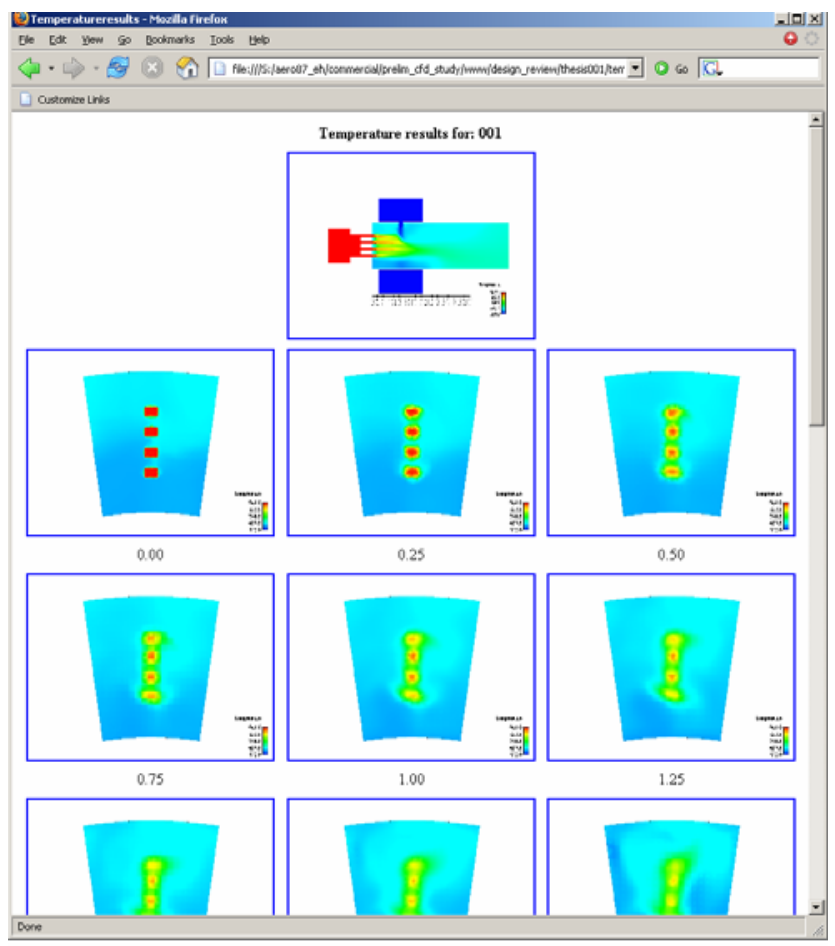

Figure 4-24 DRT: Temperature results page

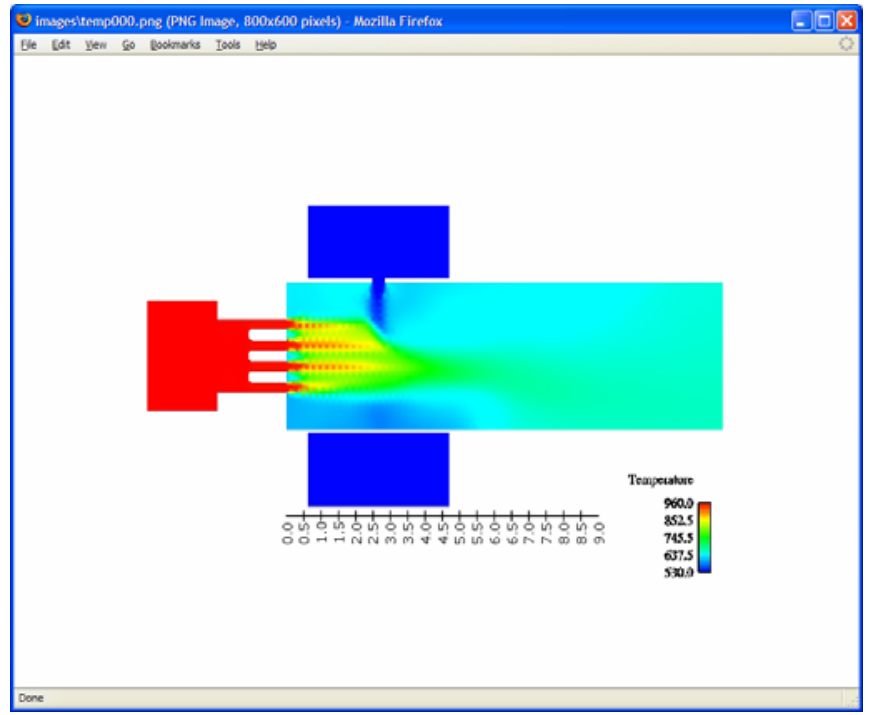

Figure 4-25 DRT: Temperature slice information image

The objective section, shown in Figure 4-26, provides a visual representation of the how quick the two streams are mixing and the temperature ranges of the flow as it 
progresses downstream. On the left hand side of the page, Figure 4-25 is shown to provide a reference of where the temperatures were calculated. On the right hand side of the page a graph is displayed to shown the maximum and minimum temperature in the flow field. By clicking on the graph an enlarged image of the graph is displayed.

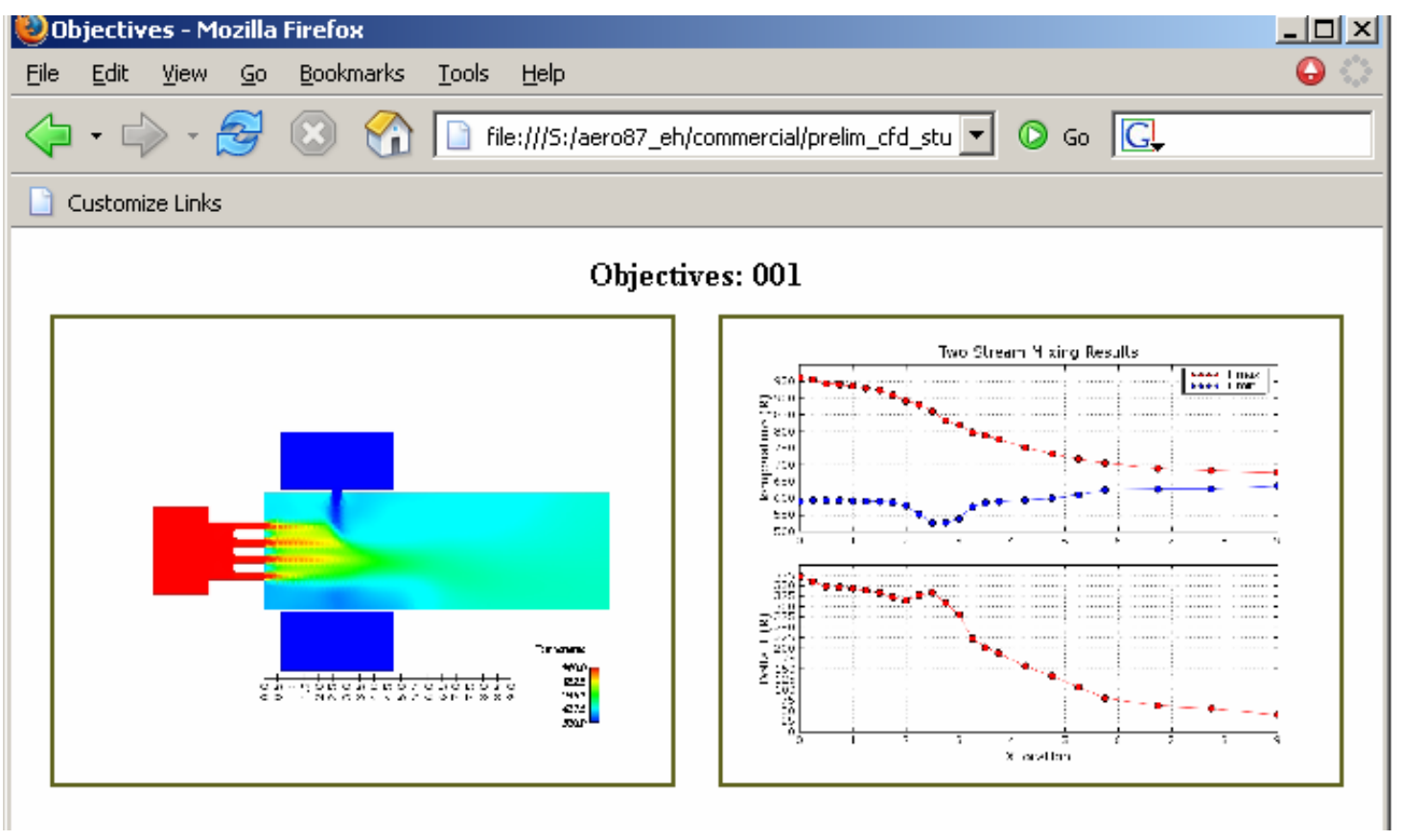

Figure 4-26 DRT: Objective section 


\section{DISCUSSION OF RESULTS}

This section presents the results of the CAD model, geometry processor, and the grid generation as applied to the conceptual study of the two stream mixing problem. The application of the method resulted in a process that could be used to analytically screen many different concepts quickly and efficiently. The results of the grid automation process and the Design Review Tool (DRT) are also presented.

To better understand the work done in this thesis to automate the grid generation process one must compare it to the current process in industry. As discussed in the introduction, this process is time consuming, costly, and involves multiple people. Automation can help eliminate these problems.

\subsection{Results: Automated Grid Generation of Parametric Models}

Automating the CAD-to-Grid process for parametric models was aided by the creation of the Master Cross Sectional Sketches (MCSS) and component modeling techniques. When these steps were added, the air solid assembly came together quickly and allowed for quick interchangeability of components. A standard process was then captured in a script to generate the grid for each new configuration. The following sections will discuss how this method improves on the current process. 


\subsubsection{Results: MCSS}

The MCSS was key in defining the critical assembly requirements. These requirements being the injection point of the two mixing streams, the mixing chamber height and length. Creating the MCSS required around an hour, but provided the parametric framework for the different concepts. The MCSS ensured that, each component in the assembly aligned properly. This approach added an extra step to the standard practices used today, but reduced the time required to assemble each component to around 15 seconds and ensured they were interchangeable.

\subsubsection{Results: Component Modeling}

Each concept was constructed using geometric entities referenced from the MCSS. This allowed for the concepts to have common entities and datums. In addition, tagging at the component level for the grid generation phase made it possible to interchange them in the assembly, which removed and added the necessary tags for the grid input file. When compared with the standard practice this takes upfront thought and time, but the payoff comes in the interchangeability of components in this and other potential assembly models.

\subsubsection{Results: Assembly Model}

Using the MCSS as a framework, the assembly model was able to swap in and out the different front ends and liners. The assembly model used to generate all of the different cases is shown in Figure 5-1. Note the components shown in Figure 5-1 are front-end concept \#1 with triangular holes in the liners that are located off of the periodic 
surfaces. Selected cases were run, which show the flexibility of the model, with different front-end concepts and hole patterns arrangements as shown in Figure 5-2 and Figure 5-3.

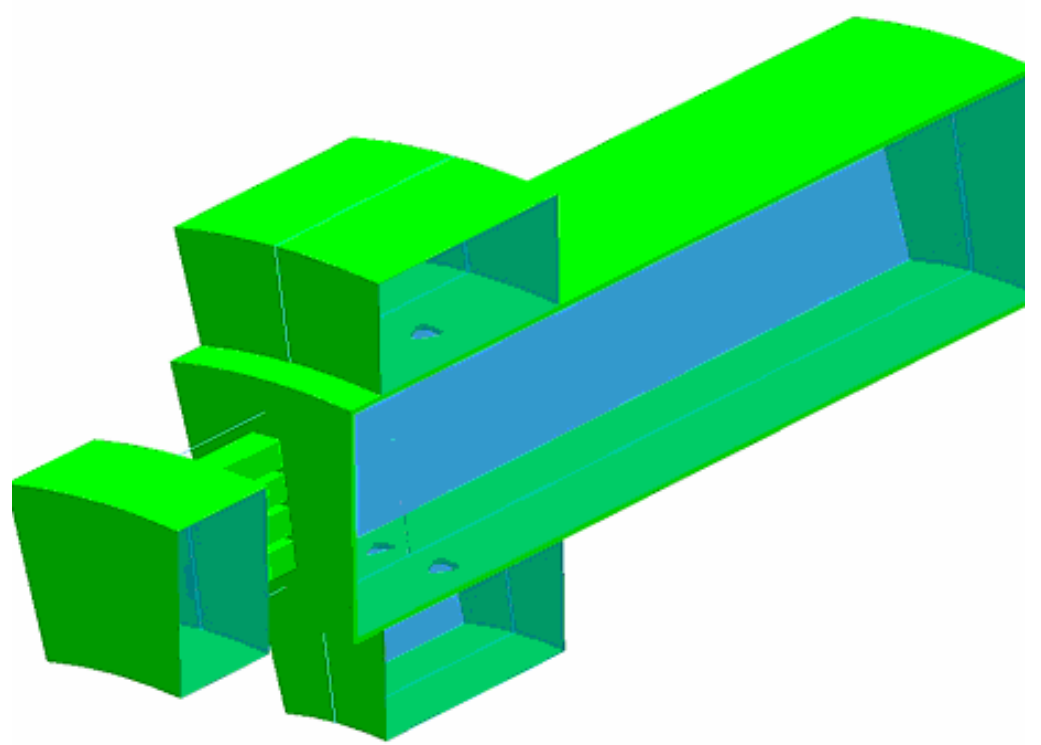

Figure 5-1 Base assembly with front-end concept \#1 and liner hole concept \#3

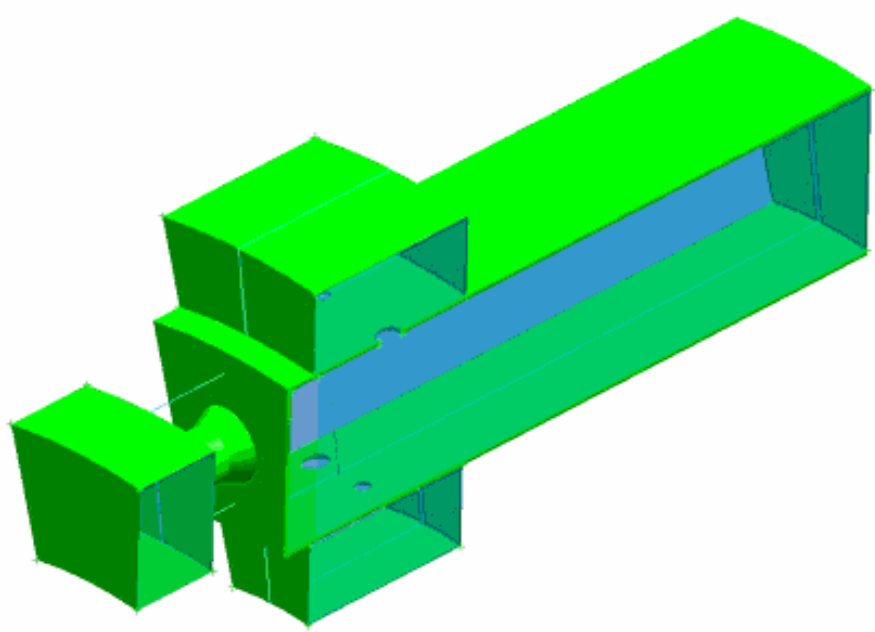

Figure 5-2 Case 002 air solid assembly with front-end concept \#2 and liner hole concept \#1 


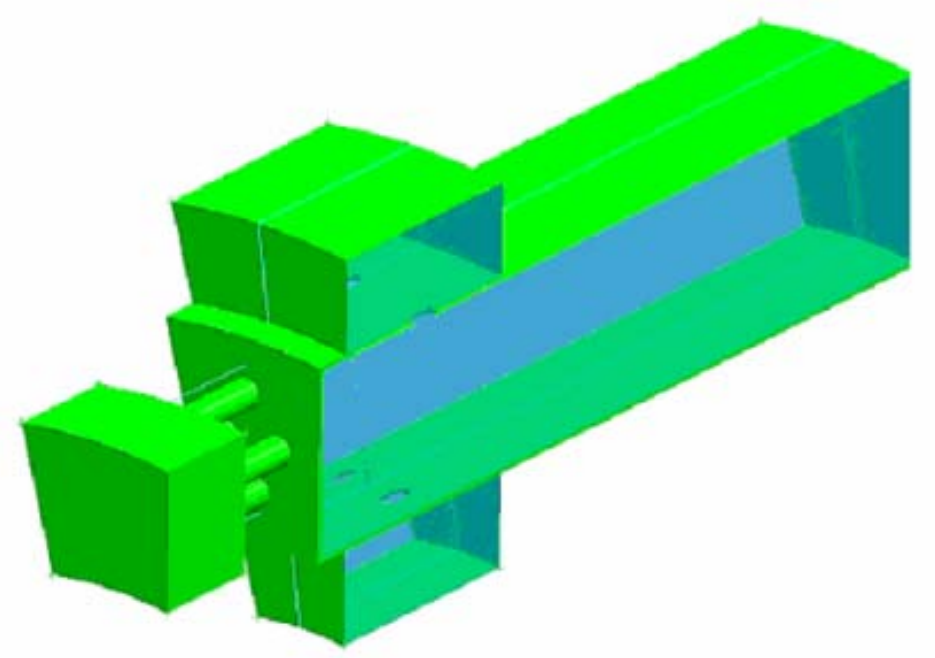

Figure 5-3 Case 006 air solid assembly with front-end concept \#3 and liner hole concept \#2

Contrast this with today's standard process of placing the different components in an assembly with the proper mating conditions. Today's approach often requires creative solutions to remove any slivers created from misalignment of the different components in the assembly. These solutions are generally done quickly to fix the model with little thought of reusability. Therefore, when changes are made down to the assembly, one or more features would likely fail.

Each concept similar to the ones above was processed using the geometry processor. This ensured that specified families in the input file were tagged and saved in the proper directory. All of the nine processed models resulted in valid input files for ICEM without any human intervention. Had this been done using standard practices, it would have required four to eight hours to modify and tag the nine models for ICEM vs the 2 minutes per model required for the geometric processor. 


\subsubsection{Grid Generation}

Using the scripting capabilities of ICEMCFD the grid generation step was automated. The script opened up the CAD model and extracted the tagged geometry. Each input file was then loaded into ICEMCFD. The input file that was automatically generated and imported into ICEM for case004 is shown in Figure 5-4.

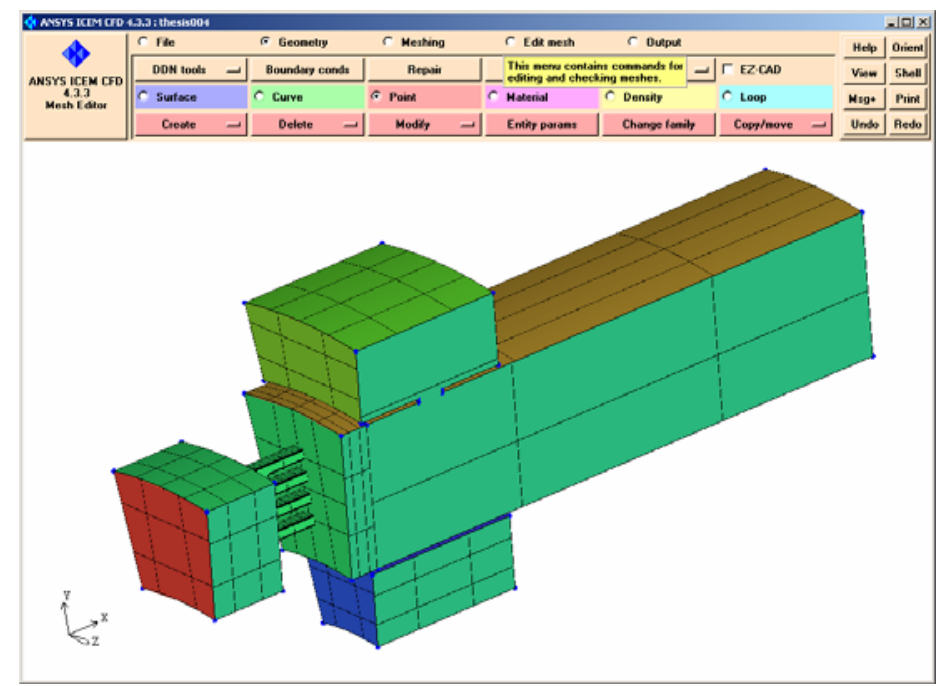

Figure 5-4 ICEM screen capture of the input file that was imported from Unigraphics

From the input file a grid was then created and smoothed using the script. A slice through the center of the grid that was generated automatically is shown in Figure 5-5. 


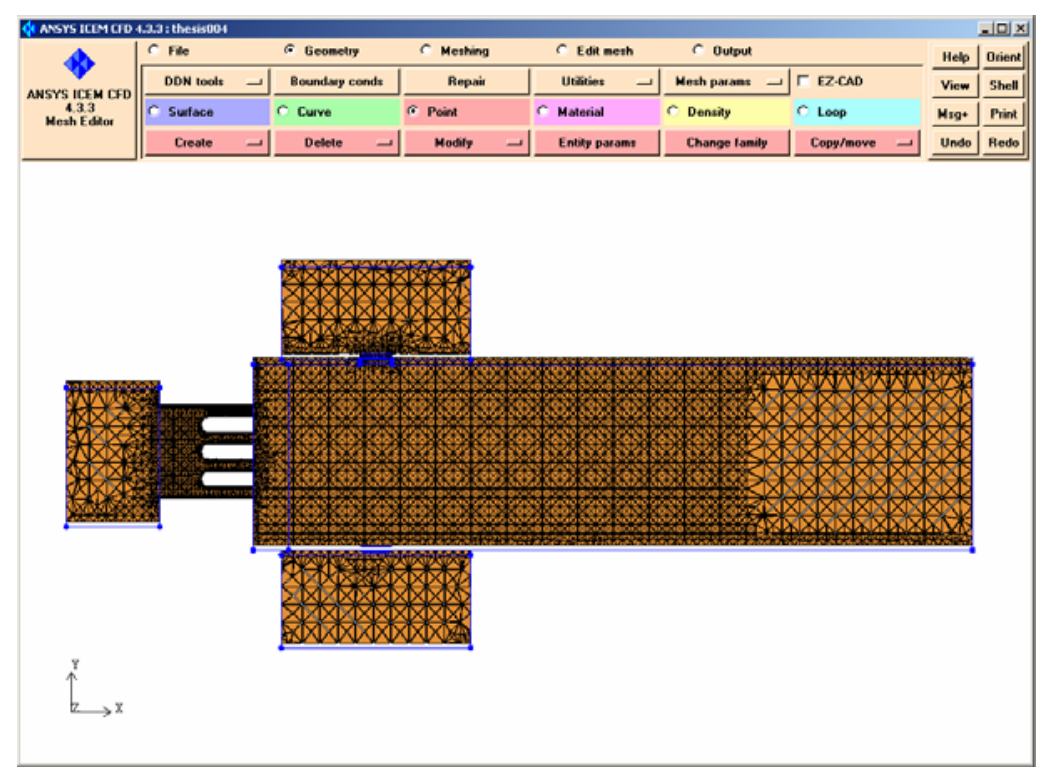

Figure 5-5 The finished grid that was generated for case004

To import the geometry, generate the grid, smooth it, and save it for inspection took around fifteen minutes. Standard practices would have required at least five to ten more minutes along with a human in the loop. Past experience has shown that it is necessary to check each grid to verify that the grid is error free of non-manifold elements, uncovered faces, low quality elements, etc. However, for the simplified geometry looked at in this thesis, all of the nine grids generated were error free.

To develop the tools used in this thesis took around 400-500 hours. However, once they are developed, they can be applied to other studies with little additional development time. In Table 5-1 is shown a comparison between the current process and the one used in this thesis. As shown in Table 5-1 the new approach takes a bit more time in the modeling set up for the first model than the current approach. However, it took $40 \%$ of the current approach to generate nine models and review them. 
Table 5-1 Current approach vs New approach

\begin{tabular}{l|r|r|r|r|}
\hline \multicolumn{1}{|c}{ Current Practice } & \multicolumn{1}{c|}{ New Approach } \\
\hline \multicolumn{1}{|c|}{ Steps } & First Model & \multicolumn{1}{c|}{ Next 8 Models } & \multicolumn{1}{c|}{ Single Model } & Next 8 Models \\
\hline Modelling & $8-12 \mathrm{hrs}$ & $32-36 \mathrm{hrs}$ & $12-16 \mathrm{hrs}$ & $4-6 \mathrm{hrs}$ \\
\hline Grid Generation & $8-12 \mathrm{hrs}$ & $32-36 \mathrm{hrs}$ & $8-12 \mathrm{hrs}$ & $3 \mathrm{hrs}$ \\
\hline Solution time & $16 \mathrm{hrs}$ & $36^{*} \mathrm{hrs}$ & $16 \mathrm{hrs}$ & $12^{\star *} \mathrm{hrs}$ \\
\hline Report Results & $4-8 \mathrm{hrs}$ & $32-56 \mathrm{hrs}$ & $4-8 \mathrm{hrs}$ & $3 \mathrm{hrs}$ \\
\hline Totals & $36-48 \mathrm{hrs}$ & $132-164 \mathrm{hrs}$ & $40-52 \mathrm{hrs}$ & $22-24 \mathrm{hrs}$ \\
\hline \multicolumn{7}{|c}{} \\
Totals for nine solutions \\
$\begin{array}{l}\text { * Assumes that three solutions were run at a time due to the added time to create a model } \\
\text { ** All eight solutions were run together due to the decreased time to generate a model }\end{array}$ \\
\hline
\end{tabular}

\subsection{Results: Design Review Tool}

The intent of the DRT was to minimize the time it takes to document each solution. Currently, the standard practice in industry is to post process the CFD solution and generate images for a set of parameters. The analyst then proceeds to make a presentation. The presentation consists of a discussion of the model, a review of the boundary conditions, and pressure field, and then a review of the results such as temperature, velocities, etc. If performed manually, the time it takes to document each case can take hours. When iterations of the model are performed, the analyst commonly takes the previous presentation as a template and updates it. This process of updating the presentation results in delaying the review of the results until a formalized presentation can be made.

The DRT helped cut this time down considerably. To determine the effectiveness of the DRT the following questions are discussed. First, does the DRT present the results effectively? Second, are the results presented in a timely manner? Third, are results of each case setup to be archived for later use? 


\subsubsection{Does the DRT present the results effectively?}

The DRT provides a standard format for each case which was set up in a way that demonstrates the elements that are reviewed in industry today. This format consists of standard layout for each case. This layout was shown previously in Figure 4-18 through Figure 4-26. By using a standard format each case can be easily compared to the other cases in the study. This format can be changed as needed by the design team and quickly updated by modifying the DRT script and reprocessing the data.

\subsubsection{Are the results presented in a timely manner?}

When each case is completed, the DRT is executed. It takes around 2.5 seconds to create all of the web pages, organize the images, and create the graphs necessary to document each case. For cases with more results and images the time required to document the case would take longer. When the DRT is finished running, each completed solution was ready for review by the design team. The standard practice used in industry today ranges from one hour to a day to document each solution. The time savings of the DRT provided the engineer and design team more time to review the results and less time generating and formatting the results for review.

\subsubsection{Are results of each case setup to be archived for later use?}

The DRT was uses a common directory structure. This directory structure places the results of the different cases in a common directory. The base directory has a folder for each case. Each case folder has their results placed in this folder as well as an images folder, that contains all of the images. This enables the DRT to create web pages using a relative directory structure for each case. The relative directory structure allowed the base 
directory to be archived and retrieved in a different directory while maintaining the functionality of the index page.

To test out the archiving and retrieving capabilities of the tool the base directory was compressed and moved to hypothetical archival location. The study directory was then retrieved and moved to a different location and uncompressed. When the index page was opened, the index page worked as designed. 


\section{CONCLUSIONS}

The objective of this thesis was to show that: 1) Valid CFD grids be made in an automated way from parametric models that undergo predictable topology changes? 2) Valid CFD grids be made in an automated way when one concept is replaced with a different concept in an assembly, 3) CFD data can be evaluated more efficiently for design reviews, and 4) CFD data can be effectively documented for later use?

To achieve the first two objectives a method for going from the CAD model to a valid grid was developed which used a geometric processor to handle the predetermined topology changes. This method showed that:

- using Master Cross Sectional Sketches (MCSS) provides a common framework for the components and assembly,

- components can be tagged for analysis at the component level and used at the assembly level,

- a geometry processor can successfully update the necessary tags in a parametric models with predetermined topology changes,

- $\quad$ valid grids can be generated for CFD in an automated way, and

- more concepts can be evaluate in a timely manner 
To demonstrate the last two objectives a DRT was created. This tool proved that:

- by using a standard file structure, and file naming convention each case can be formatted for review in under 10 seconds.

- a modular structure allows sections to be modified, added, or removed in order to meet the needs of the design team, and

- the results can be archived for later use when the study is completed.

The method and tools presented in this thesis were formally verified using three different front-end and liner concepts for a two stream mixing problem. A total of nine different grids were generated to evaluate the temperature range of the two streams at different locations in the mixing chamber. The results of the method and tools were documented in the previous chapters.

It was found that by using the method outlined in this thesis that parametric models with predetermined topology changes as well as component replacement could be used to create valid grid in under an hour, where it currently takes anywhere from two hours to a week in industry. The general approach presented here will allow the aerospace, automotive, and other industries to evaluate more concepts before one concept is selected for the detailed design phase. It will also help them look at different features in the design space once the detailed design phase has begun.

The DRT was shown to be useful to present the data of nine cases for review in less than a minute and in a consistent format. It enabled the CFD analyst and design team to focus more time on the results and less time on the preparation. In addition, the data can be compared in a more consistent manner by using a standard format to review the results. This work also provides a starting point for future work in coupling the CAD tool more closely with CAE analysis tools. 


\subsection{Future Work}

There are multiple activities that would take the method outlined for this thesis to the next level. One activity would be to integrate the geometry processor, which handled predetermined topology changes (i.e. Holes on the periodics, component interchanging, new edges created on a surface) with the discrete feature work done by King (2004). Another activity would be to validate this process using more complex geometry and cases from industry.

As for the DRT more work is needed to:

1. extend the capabilities of the tool for comparing the different concepts (i.e. allowing the user to see a side by side comparison of two or more case of their choice),

2. make it more user friendly by adding a graphical user interface, and

3. make it more robust to adapt to different conceptual studies and analyses. By focusing on these areas of the DRT it will become a vital tool in the design process and help in the review and documentation of each case run in a conceptual study. 



\section{REFERENCES:}

Alonso, Juan J., Martins, Joaquim R. R. A., Reuther, James J., Haimes R., Crawford, Curran A. (2003 ) "High Fidelity Aero-Structural Design Using a Parametric CAD-Based Model” AIAA 2003-4329.

Anderl, R. and Mendgen, R. (1995) "Parametric Design and its Impact on Solid Modeling Applications” Solid Modeling, Salt Lake City, Utah, pp 1-12

Ault, Holly K. (1999) “Using Geometric Constraints to Capture Design Intent” Journal for Geometry and Graphics, vol 3, No. 1, pp 39-45.

Crocker, D. Scott, Nickolaus, Dan, Smith, Clifford E., (1998) "CFD Modeling of a Gas Turbine Combustor from Compressor Exit to Turbine Inlet” International Gas Turbine \& Aeroengine Congress \& Exhibition, Stockholm, Sweden, June 25

Cervenka, Michael, (2000) “The Rolls-Royce Trent Engine”, http://www.msm.cam.ac.uk/phase-trans/mphil/Trent1/sld014.htm

Dawes, W.N., Dhanasekaran, P.C., Demargne, A.A.J., Kellar, W.P., and Savill, A.M., (2001), "Reducing Bottlenecks in the CAD-to-Mesh-to-Solution Cycle Time to Allow CFD to Participate in Design,” Journal of Turbomachinary, Vol. 123, pp. 552-557.

Haimes, R., and Crawford, C., (2003), "Unified Geometry Access for Analysis and Design” Proceedings, $12^{\text {th }}$ International Meshing Roundtable, Santa Fe, New Mexico, Sept 14-17, 2003, pp. 21-31.

Hardee, E., Chang, Kuang-Hua, Tu, Jian, Choi, Kyung K., Grindeanu, I., (1999) “A CAD-Based design parameterization for shape optimization of elastic solids" Advances in Engineering Software, vol 30, pp 185-199.

Hoffmann, C.M., Kim, K.J., (2001), Towards valid parametric CAD models, CAD Computer Aided Design, Vol 33, Issue 1, pp 81-90.

Hogge, D., (2002), Integrating Commercial CAx Software to Perform Multidisciplinary Design Optimization, M.S. Thesis, Brigham Young University.

King, M., (2004), A CAD-centric approach to CFD analysis with discrete features, M.S. Thesis, Brigham Young University. 
Lawson, John, Erjavec, John, (2001) Modern Statistics for Engineering and Quality Improvement, Wadsworth Group, Duxbury.

Lee, K., (1999), Principles of CAD/CAM/CAE Systems, Addison Wesley, Reading, MA.

Malecki, Robert E., Rhie, Chae M., McKinney, Randal G., Ouyang, Hong, Syed, Saadat A. Colket, Meredith B., Madabhushi, Ravi K. (2001) "Application of an Advanced CFD-Based Analysis System to the PW6000 Combustor to Optimize Exit Temperature Distribution - Part 1: Description and Validation of the Analysis Tool" Proceedings of ASME: ASME TURBOEXPO 2001, New Orleans, Louisiana, June 4-7, 2001-GT-0062.

Newman III, J. C., Taylor III, A. C., Barnwell, R. C., Newman, P. A., Hou, G., (1999) "Overview of Sensitivity Analysis and Shape Optimization for Complex Aerodynamic Configurations”, Journal of Aircraft, vol 36., No 1. JanuraryFebruary

Rohm III, T., (2001) Graphical Creation of CAD Parametric Application Programs, M.S. Thesis, Brigham Young University.

Rohm III, T., Jones, C.,, Tucher, S., Jensen, C., (2000), "Parametric Engineering Design Tools and Applications,” ASME Design Automation Conference, Baltimore, MD, September, 10-13, 2000, pp.657-664.

Samareh, J. A., (1999) "Status and Future of Geometry Modeling and Grid Generation for Design and Optimization,” Journal of Aircraft, vol. 36, pp. 97104.

Smith, R.J., Leshchziner, M.A., (1996), Automatic grid generation for complex geometries, Aeronautical Journal, Vol. 100, Issue 991, pp. 7-14.

Srinivasan, H., Jensen, C. G., Kopper, F. C., Staubach, J. B., and Pack, D. R., "Assembly Parametrics for Multidisciplinary Design Optimization" $8^{\text {th }}$ International Society for Productivity Enhancement Conference on Concurrent Engineering, Anaheim, CA, July 29 - August 1, 2001, pp. 158-165

Subrahmanyam, S., DeVries W., and Pratt, M., (1995), "Feature Attributes and their Role in Product Modeling," Proceedings of the Symposium on Solid Modeling and Applications, Salt Lake City, UT, May 17-19, 1995 pp. 115-124.

Tangirala, Venkat E., Tolpadi, Anil K., Danis, Al M., Mongia, Hukam, (2000) "Parametric Modeling Approach to Gas Turbine Combustor Design" Proceedings of ASME: ASME TURBOEXPO 2000, May 8-11 Munich Germany, 2000-GT-129 
Ulrich, Karl., Epinger, Steven., (2000) Product Design and Development, McGrawHill. 


\section{APPENDIX}




\section{Appendix A: Setup information and flow calculations}

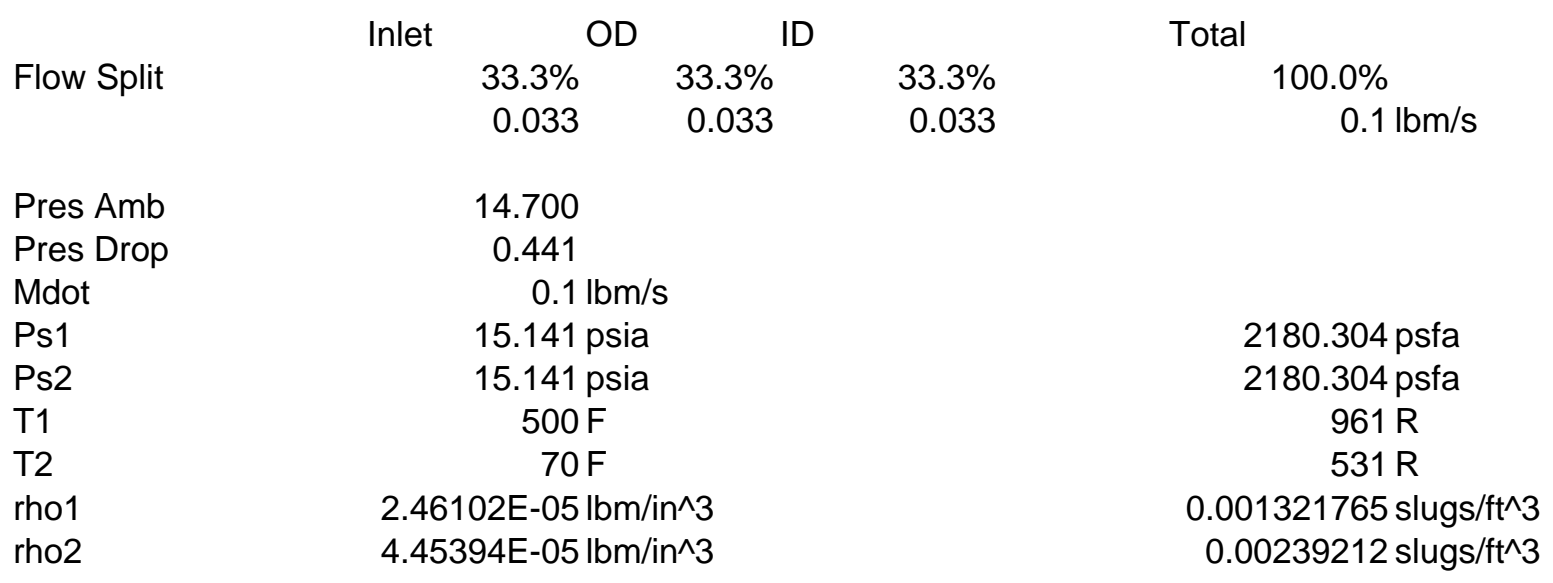

\begin{tabular}{|l|r|r|r|r|r|r|}
\hline \multicolumn{7}{|c|}{ Boundary Conditions } \\
\hline BC Name & BC Tag & BC Type & Area & P & Rho & Vel (ft/s) \\
\hline FE Inlet & 1 inlet-speed & 10.282 & 15.1410 & $2.46 \mathrm{E}-05$ & 10.9780 \\
\hline Exit & 2 & exit & 13.7090 & 14.7000 & $3.79 \mathrm{E}-05$ & 16.0404 \\
\hline Periodic & 11 periodic-a & & & & \\
\hline Periodic & 12 & periodic-b & & & & \\
\hline ID Inlet & 20 inlet-speed & 5.0840 & 15.1410 & $4.45 \mathrm{E}-05$ & 12.2673 \\
\hline OD Inlet & 21 inlet-speed & 8.6250 & 15.1410 & $4.45 \mathrm{E}-05$ & 7.2309 \\
\hline Wall & 600 moving-wall & & & & \\
\hline Wall & 620 moving-wall & & & & \\
\hline Wall & 630 moving-wall & & & & \\
\hline Wall & 640 & moving-wall & & & & \\
\hline
\end{tabular}

Acd $=$ measured equivalent area of the burner liner $\left(\mathrm{in}^{\wedge} 2\right)$

$\mathrm{g}=$ unit conversion factor, $32.2 \mathrm{lbm} * \mathrm{ft} / \mathrm{lbf}^{*} \mathrm{~s}^{\wedge} 2$

Delta $\mathrm{P}=$ measured pressure drop across the combustor liner (psid)

$\mathrm{T}=$ Combustor inlet temperature, $\operatorname{deg} \mathrm{F}$

$\mathrm{P}=$ Static pressure at the combustor liner inlet, psia

mdot $=\operatorname{sqrt}(2 * 32.2 / 144)^{\star} \mathrm{ACd}{ }^{*} \mathrm{sqrt}\left(\mathrm{rho}^{*}\right.$ deltaP $)(\mathrm{lbm} / \mathrm{s})$

rho $=2.698825^{\star} \mathrm{P} /(\mathrm{T}+459.67)\left(\mathrm{lbm} / \mathrm{ft}^{\wedge} 3\right)$

\begin{tabular}{|l|r|r|r|c|}
\hline \multicolumn{5}{|c|}{ Flow Area Calculations } \\
\hline & OD & ID & FE & Units \\
\hline ACd & 0.271 & 0.271 & 0.364 & $\left(\right.$ in^$\left.^{\wedge}\right)$ \\
\hline delP & 0.441 & 0.441 & 0.441 & $\mathrm{psi}$ \\
\hline Rho & 0.077 & 0.077 & 0.043 & $\mathrm{lbm} / \mathrm{ft}$ ^3 \\
\hline Mdot & 0.033 & 0.033 & 0.033 & $\mathrm{lbm} / \mathrm{s}$ \\
\hline
\end{tabular}

\begin{tabular}{|l|r|r|r|}
\hline \multicolumn{4}{|c|}{ Initial Guesses For Discharge Coefficient } \\
\hline & Circle & Ellipse & Triangle \\
\hline Cd & 0.7 & 0.65 & 0.6 \\
\hline Geom Area & 0.387 & 0.416 & 0.451 \\
\hline & & & \\
\hline
\end{tabular}




\begin{tabular}{|l|r|r|r|} 
& 4 Slots & Cylinder & 4 Cylinders \\
\hline Cd & 0.75 & 0.8 & 0.95 \\
\hline Geom Area & 0.485 & 0.455 & 0.383 \\
\hline
\end{tabular}

\begin{tabular}{|r|r|r|r|}
\hline \multicolumn{5}{|c|}{ Concept 1 ACD calculations } \\
\hline \multicolumn{1}{|c|}{ Target ACd } & Feom Area & \multicolumn{1}{c|}{ Cd } \\
\hline 0.33 & 0.307 & 0.357 & 0.860 \\
\hline 0.364 & 0.339 & 0.394 & 0.861 \\
\hline 0.4 & 0.370 & 0.432 & 0.856 \\
\hline & & & 0.86 \\
\hline Target ACd & & & \multicolumn{1}{c|}{ Ld } \\
\hline 0.24 & 0.246 & 0.324 & 0.757 \\
\hline 0.271 & 0.273 & 0.382 & 0.714 \\
\hline 0.3 & 0.294 & 0.423 & 0.694 \\
\hline
\end{tabular}

\begin{tabular}{|r|r|r|r|}
\hline \multicolumn{5}{|c|}{ Concept 2 ACD calculations } \\
\hline \multicolumn{1}{|c|}{ Target ACd } & \multicolumn{1}{c|}{ FE } & Geom Area & \multicolumn{1}{c|}{ Cd } \\
\hline 0.33 & 0.354 & 0.384 & 0.922 \\
\hline 0.364 & 0.354 & 0.384 & 0.922 \\
\hline 0.4 & 0.354 & 0.384 & 0.922 \\
\hline & & & \\
\hline Target ACd & Liner & Geom Area & \multicolumn{1}{c|}{ Cd } \\
\hline 0.24 & 0.279 & 0.369 & 0.755 \\
\hline 0.271 & 0.304 & 0.417 & 0.728 \\
\hline 0.3 & 0.324 & 0.462 & 0.702 \\
\hline
\end{tabular}

\begin{tabular}{|r|r|r|r|}
\hline \multicolumn{6}{|c|}{ Concept 3 ACD calculations } \\
\hline \multicolumn{1}{|c|}{ Target ACd } & \multicolumn{1}{c|}{ FE } & Geom Area & \multicolumn{1}{c|}{ Cd } \\
\hline 0.33 & 0.239 & 0.330 & 0.724 \\
\hline 0.364 & 0.264 & 0.364 & 0.726 \\
\hline 0.4 & 0.291 & 0.400 & 0.729 \\
\hline & & & \\
\hline Target ACd & Liner & Geom Area & Cd \\
\hline 0.24 & 0.267 & 0.345 & 0.772 \\
\hline 0.271 & 0.293 & 0.397 & 0.738 \\
\hline 0.3 & 0.322 & 0.445 & 0.722 \\
\hline
\end{tabular}




\section{Appendix B: DRT Scripts}

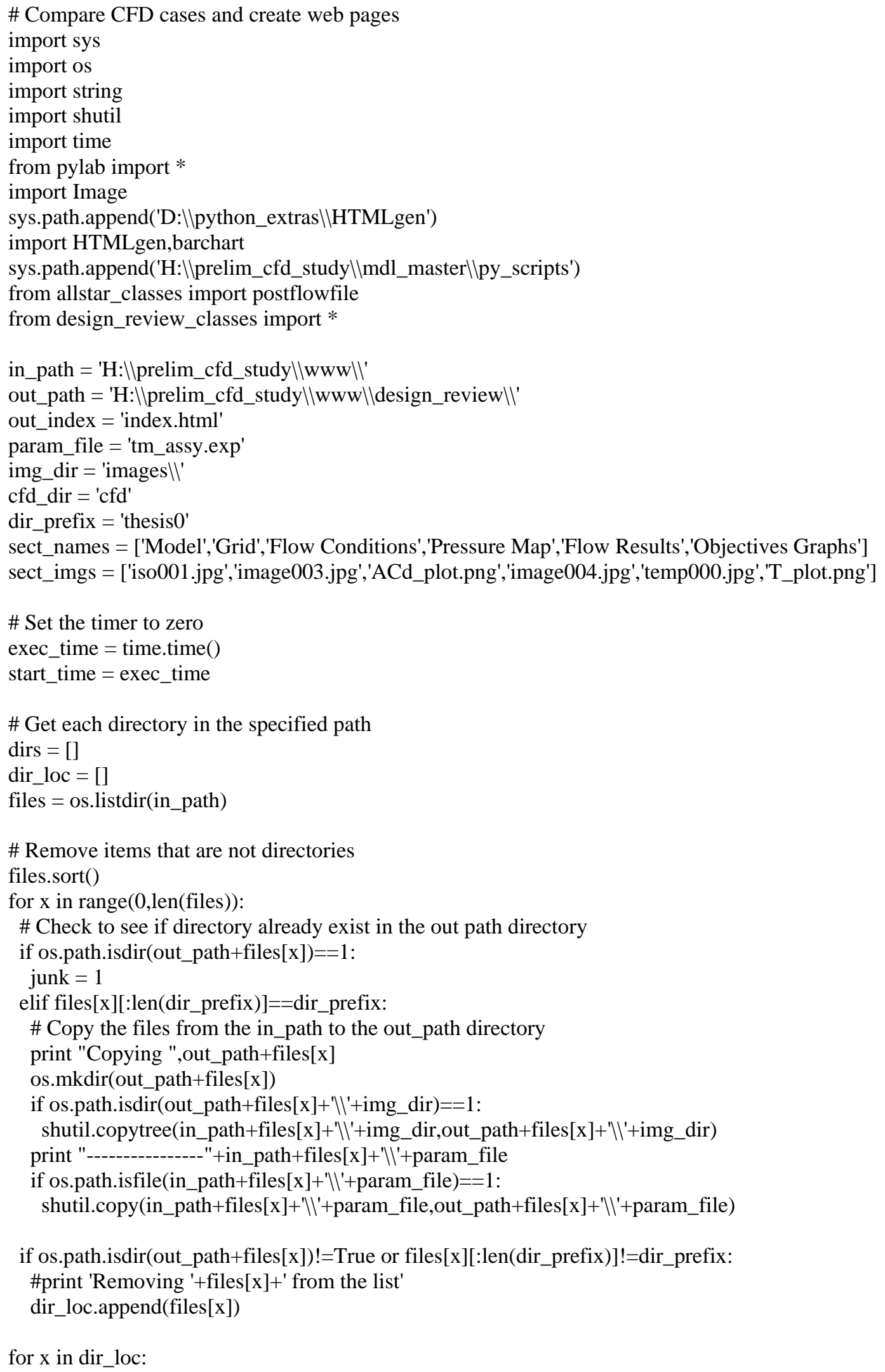


files.remove(x)

for $\mathrm{x}$ in range(0,len(files)):

exec_time $=$ time.time ()

if os.path.isdir(out_path+files[x]):

\# Gather the data from the allstar header file

ci = case_info(in_path+files[x]+'|l'+cfd_dir+'I|')

ci.get_info()

\# Create indexes of the results

if os.path.isdir(out_path+files[x]+'|l'+img_dir)==1:

case_doc $=$ HTMLgen.SimpleDocument(title $=$ files $[\mathrm{x}])$

case_table1 $=$ HTMLgen.Table(border=0, width=600, cell_align="center",

tabletitle $=$ 'Configuration '+files[x][-

3:],heading_align='center',heading_valign='middle',column1_align='center')\#,colspan=[2,1])

case_table $=$ HTMLgen.Table(border=0, cell_align="center", heading $=[$ 'Configuration '+files[x][-

3:]+' Case Info'])

case_table.body.append(['Iterations: '+str(ci.iterations)])

case_table.body.append(['Elements: '+str(ci.elements)])

case_table.body.append([HTMLgen.Href(param_file,"Parameters")])

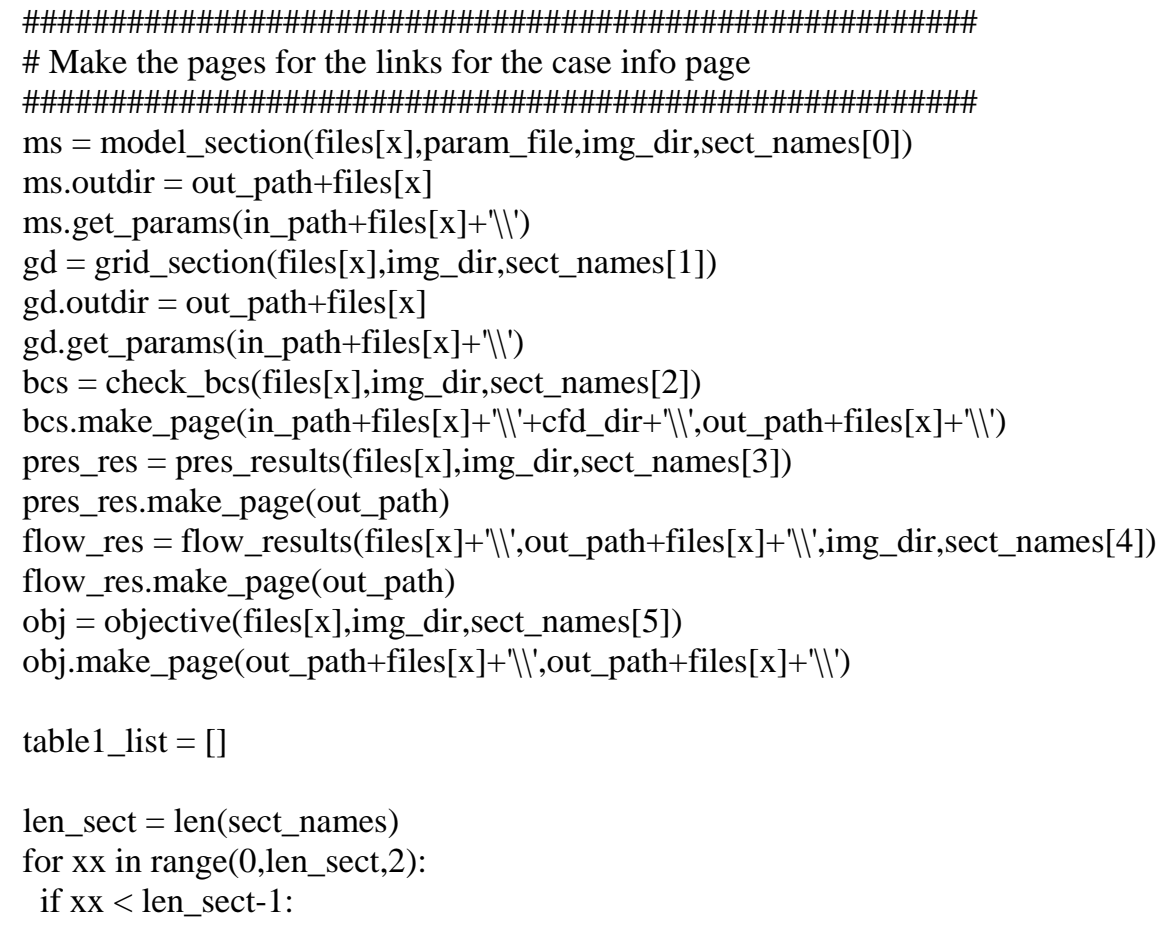

table1_list.append([HTMLgen.Href(sect_names[xx]+'.html',HTMLgen.Image(img_dir+'/'+sect_imgs[x x],width='300',height='225')),HTMLgen.Href(sect_names[xx+1]+'.html',HTMLgen.Image(img_dir+'//'

+sect_imgs[xx+1],width='300',height='225'))]) table1_list.append([sect_names[xx],sect_names[xx+1]]) else:

table1_list.append([HTMLgen.Href(sect_names[xx]+'.html',HTMLgen.Image(img_dir+'/'+sect_imgs[x x],width='300',height='225'))]) table1_list.append([sect_names[xx]]) 


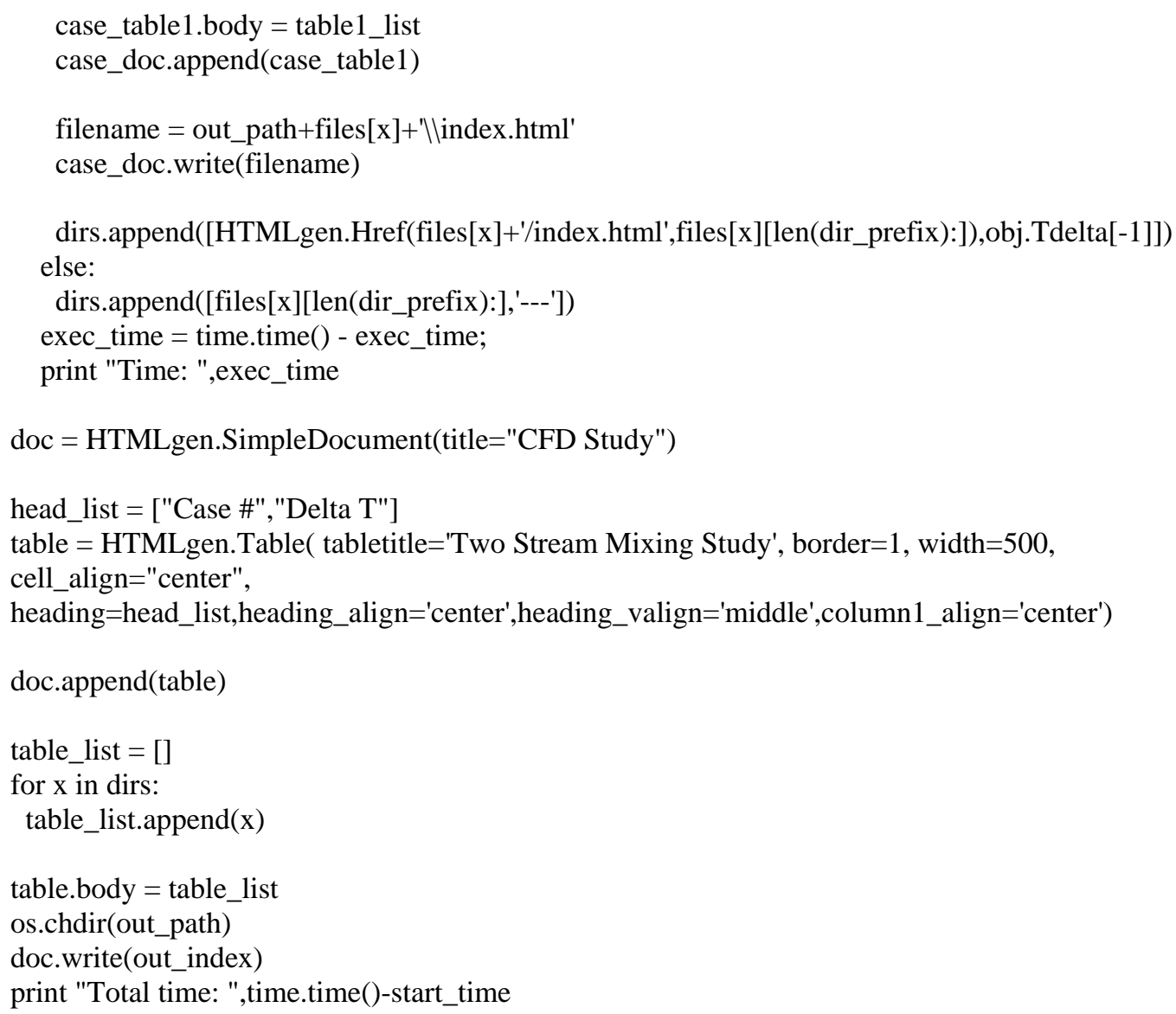




\section{Modules used by the DRT}

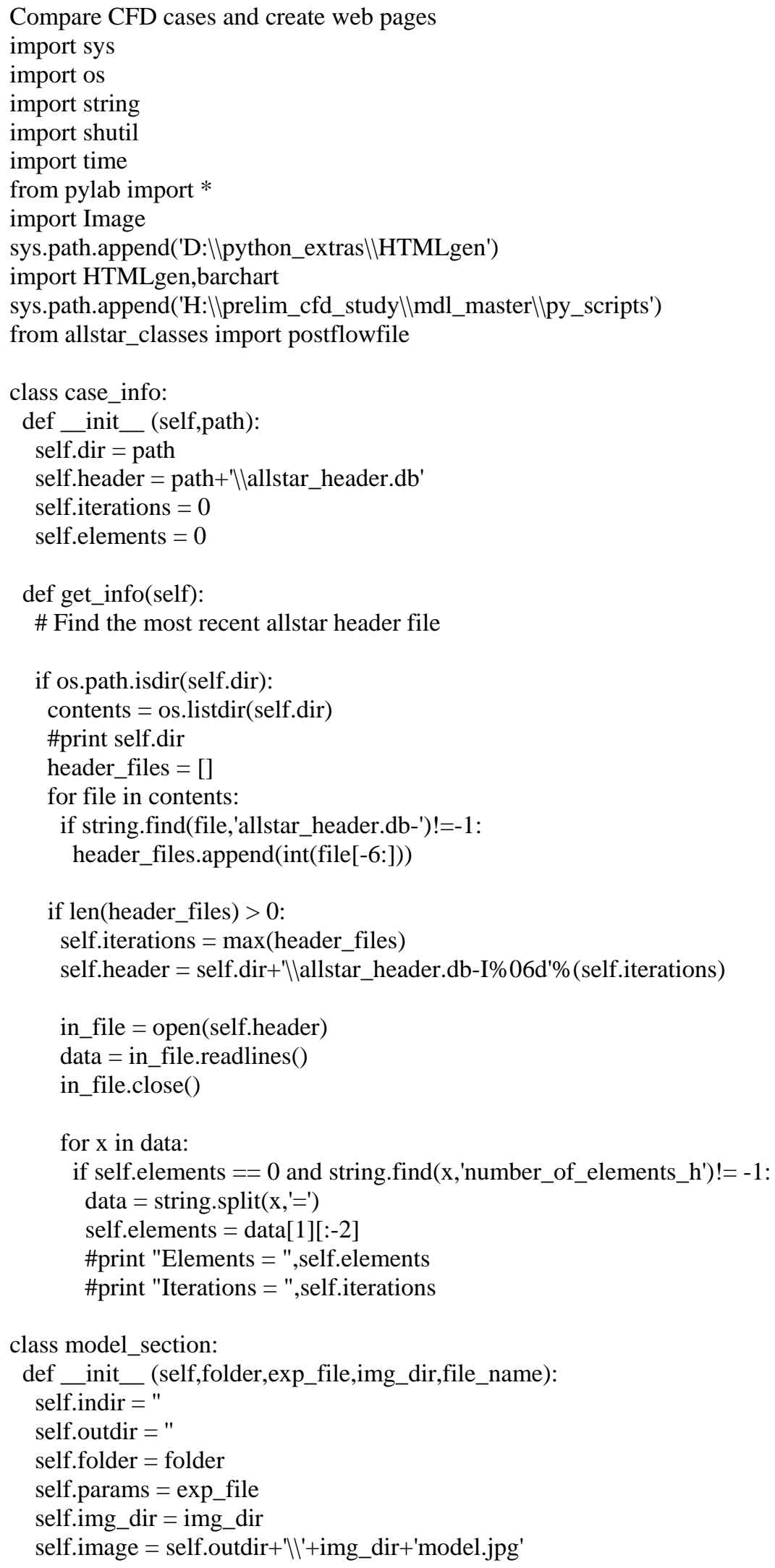




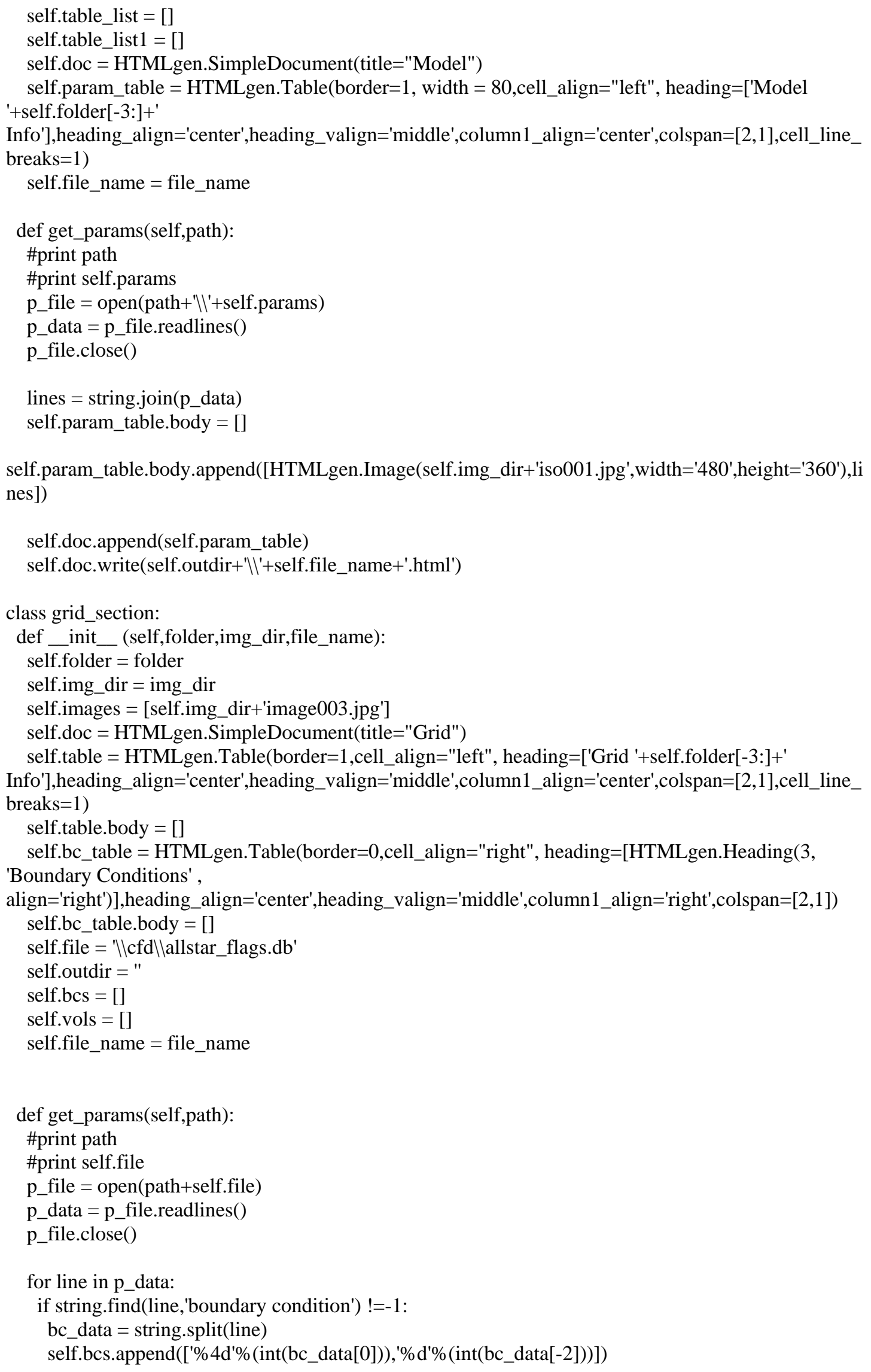




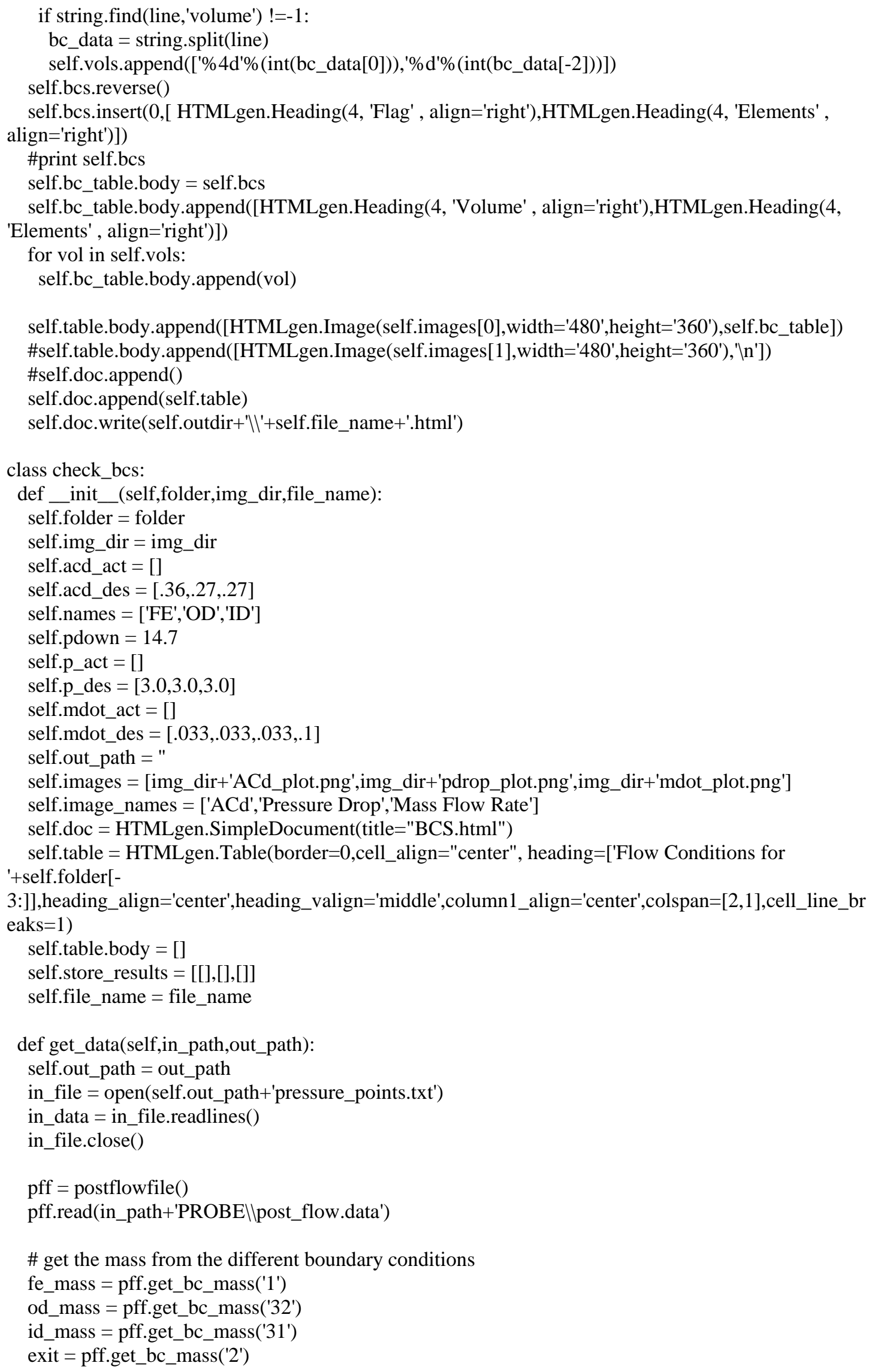


self.mdot_act.append(fe_mass)

self.mdot_act.append(od_mass)

self.mdot_act.append(id_mass)

self.mdot_act.append(fe_mass+od_mass+id_mass)

\# Calculate the pressure drops

in_data $=$ in_data[2:]

cham_ps $=0.0$

for $x$ in range $(0,4)$ :

cham_ps = cham_ps + float(string.split(in_data[x])[0])/144.0

cham_ps = cham_ps/(4.0)

\#print cham_ps

od_pt $=0.0$

for $x$ in range $(4,6)$ :

od_pt $=$ od_pt + float(string.split(in_data[x])[1])/144.0

od_pt $=$ od_pt $/ 2.0$

id_pt $=0.0$

for $\mathrm{x}$ in range(6,8):

id_pt $=$ id_pt + float(string.split(in_data[x])[1])/144.0

id_pt $=$ id_pt $/ 2.0$

fe_pt $=0.0$

for $x$ in range $(8,10)$ :

$\mathrm{fe} \_\mathrm{pt}=\mathrm{fe} \_\mathrm{pt}+$ float(string.split(in_data[x])[1])/144.0

fe_pt $=$ fe_pt $/ 2.0$

self.p_act.append(100.0*(fe_pt/cham_ps-1.0))

self.p_act.append(100.0*(od_pt/cham_ps-1.0))

self.p_act.append(100.0*(id_pt/cham_ps-1.0))

\#print self.p_act

\#calculate the ACd for each component

fe_acd = fe_mass/(1.099*sqrt(cham_ps*(fe_pt-cham_ps)/pff.get_bc_temp(1)))

od_acd $=$ od_mass $/(1.099 *$ sqrt(cham_ps*(od_pt-cham_ps)/pff.get_bc_temp(32)) $)$

id_acd = od_mass/(1.099*sqrt(cham_ps*(id_pt-cham_ps)/pff.get_bc_temp(31)))

\# add the acd values to the list

self.acd_act.append(fe_acd)

self.acd_act.append(od_acd)

self.acd_act.append(id_acd)

\# write the values to a file

post_file = open(out_path+'bc_check.txt','w')

post_file.write("ACd Values\n------------------ $\backslash n$ ")

for $\mathrm{x}$ in range(0,len(self.acd_act)):

post_file.write(self.names[x]+'ไt'+str(self.acd_act[x])+' $\mid \mathrm{n}$ ')

post_file.write("'nPressure Drop Values $\backslash n$------------------ $\backslash n$ ")

for $x$ in range(0,len(self.p_act)):

post_file.write(self.names[x]+'ไt'+str(self.p_act[x])+' $\backslash n ')$

post_file.write("'nMass Flow Values $\backslash n$------------------'n")

for $\mathrm{x}$ in range(0,len(self.names)):

post_file.write(self.names[x]+' $\backslash \mathrm{t}^{\prime}+\operatorname{str}($ self.mdot_act $\left.[\mathrm{x}])+{ }^{\prime} \backslash \mathrm{n}^{\prime}\right)$

post_file.close() 


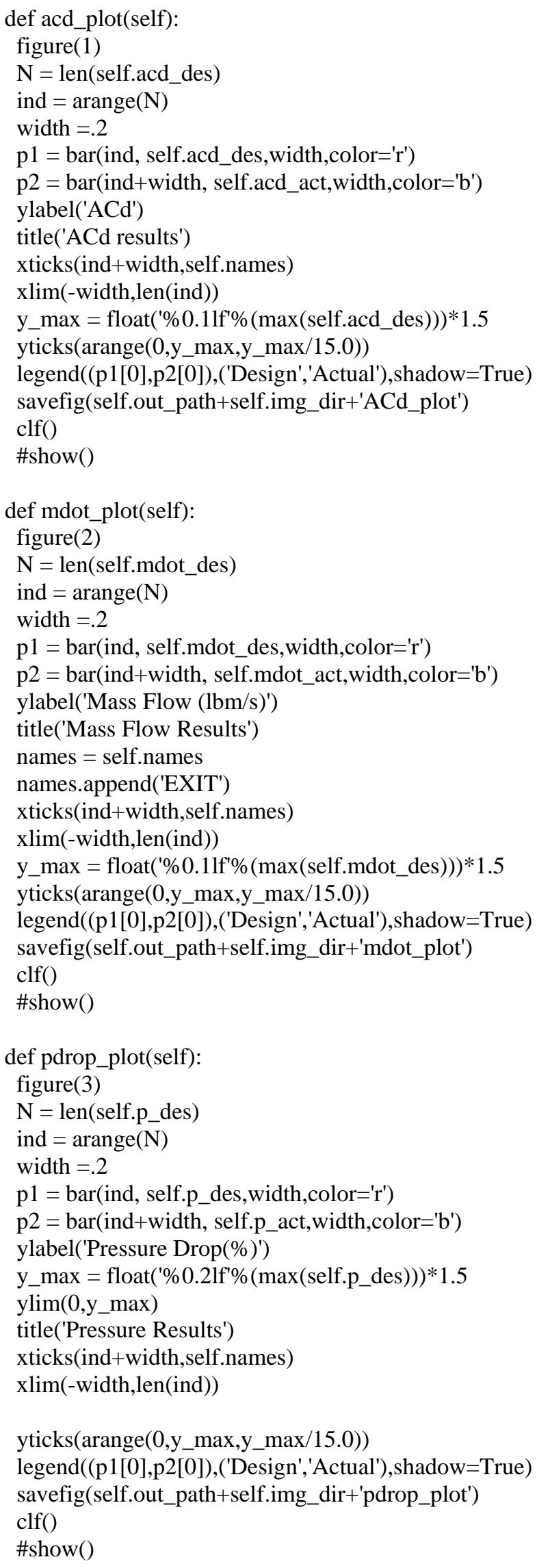




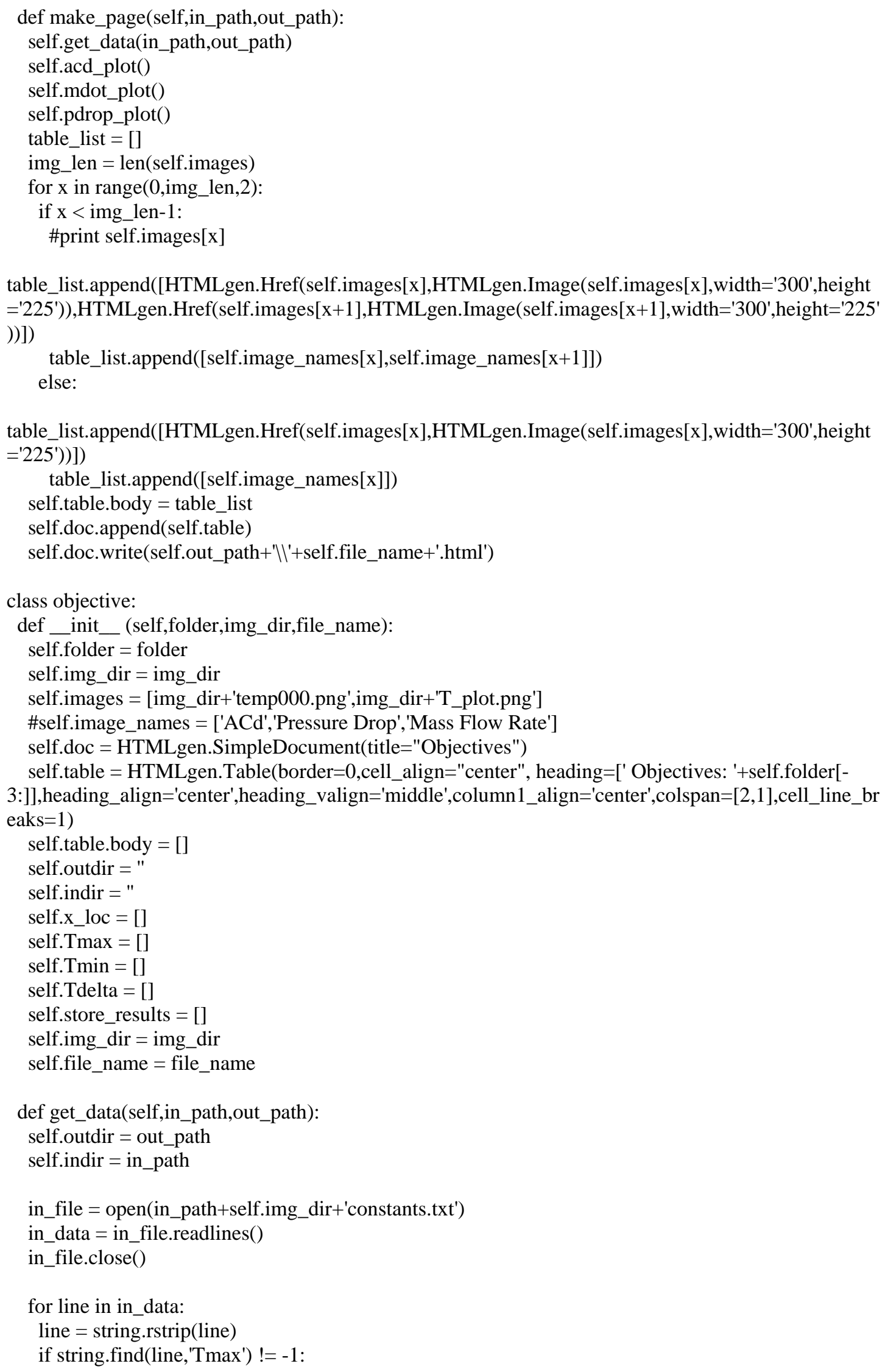




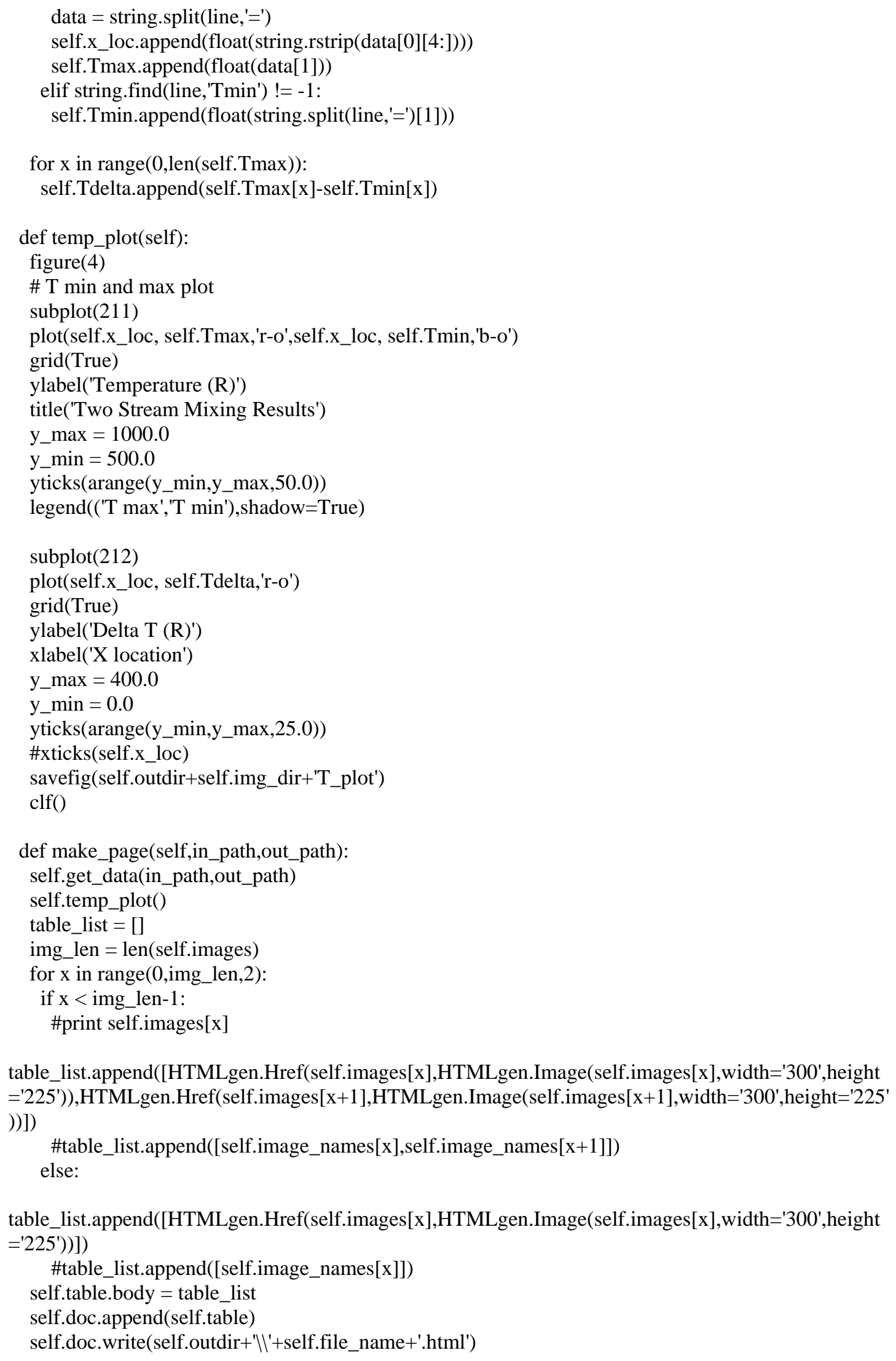




\section{Appendix C: DRT Screen Grabs For Concept 001}

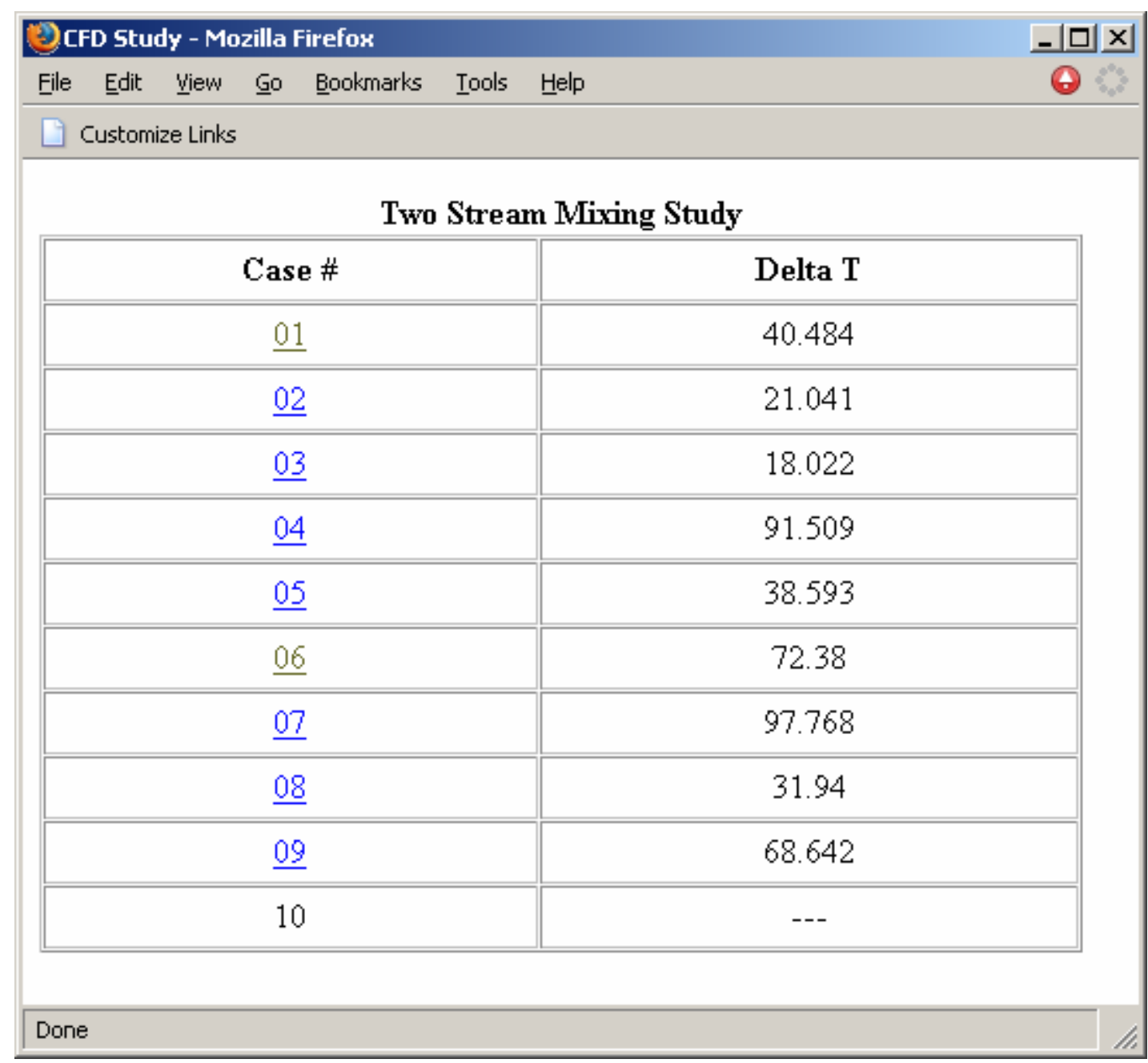

Index Page 


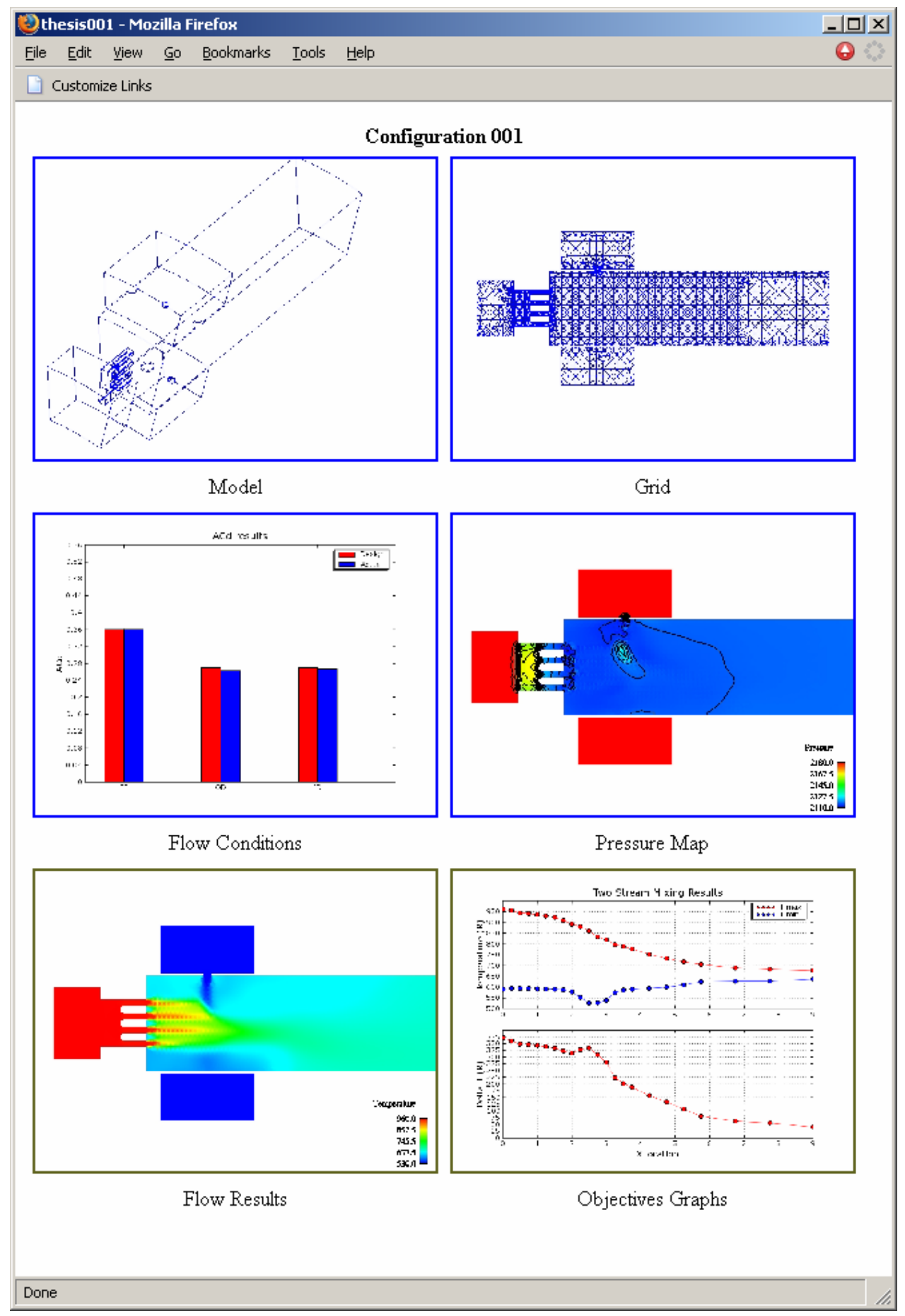

Case Review Page 


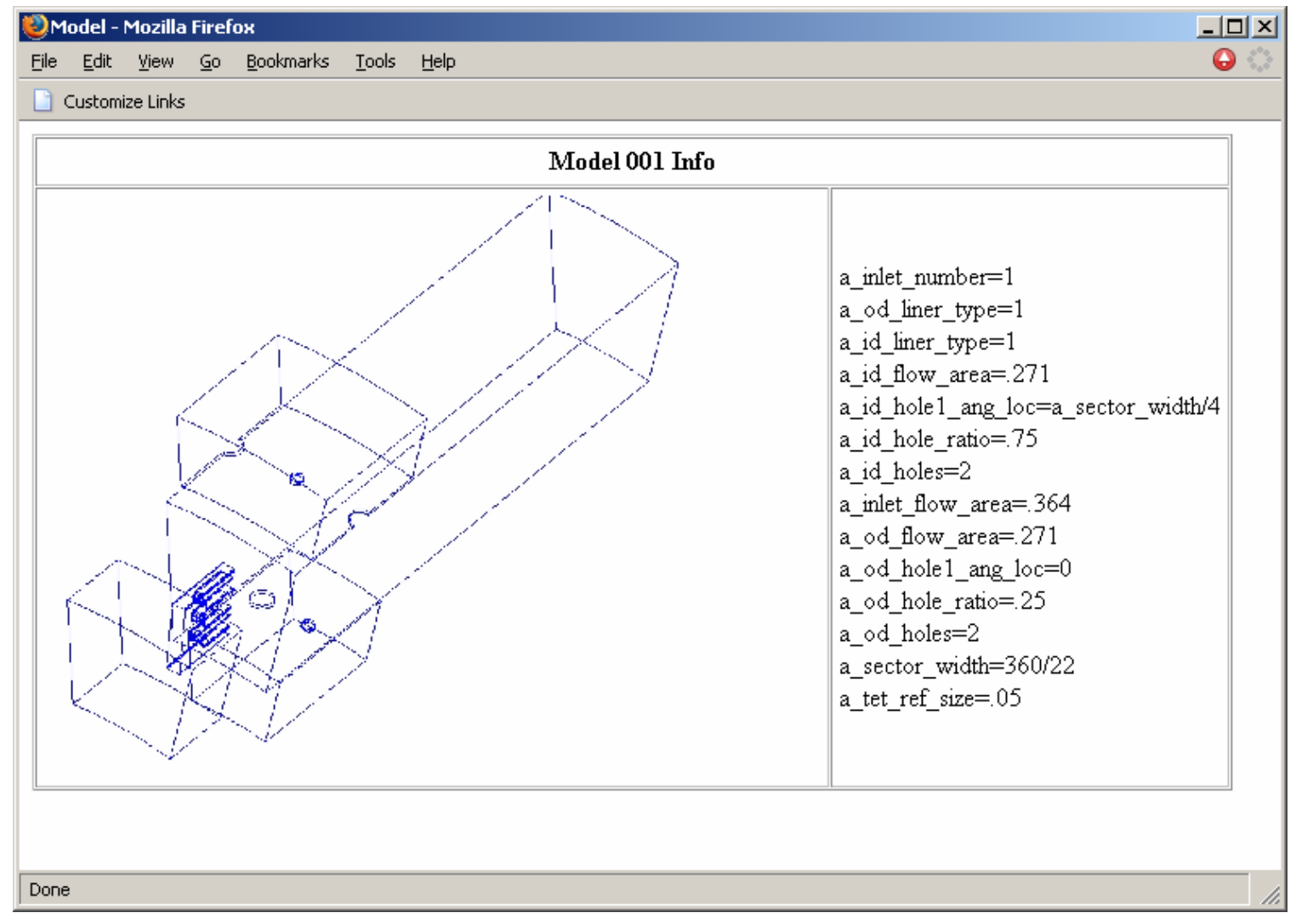

Model Section 


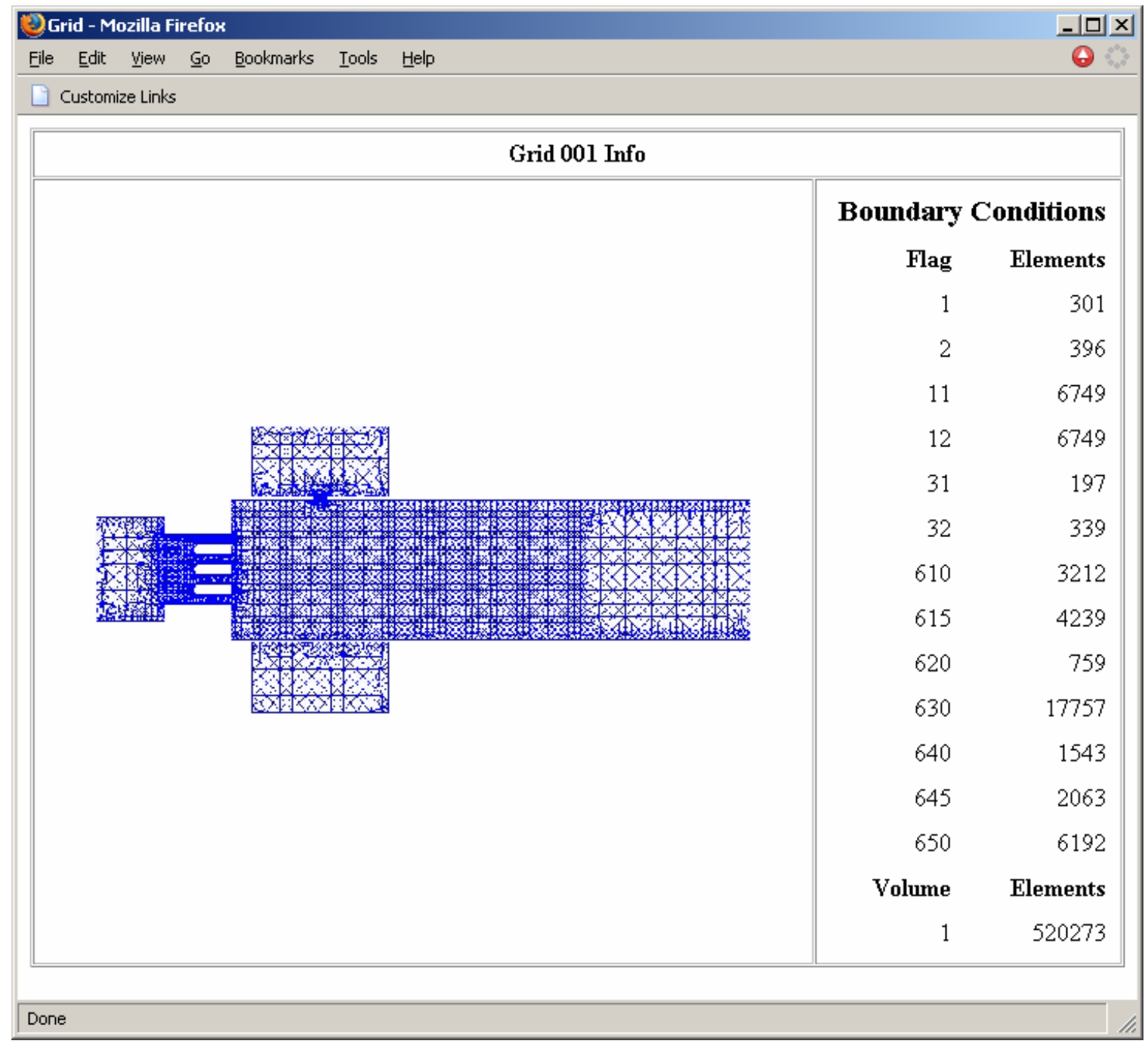

Grid Section 


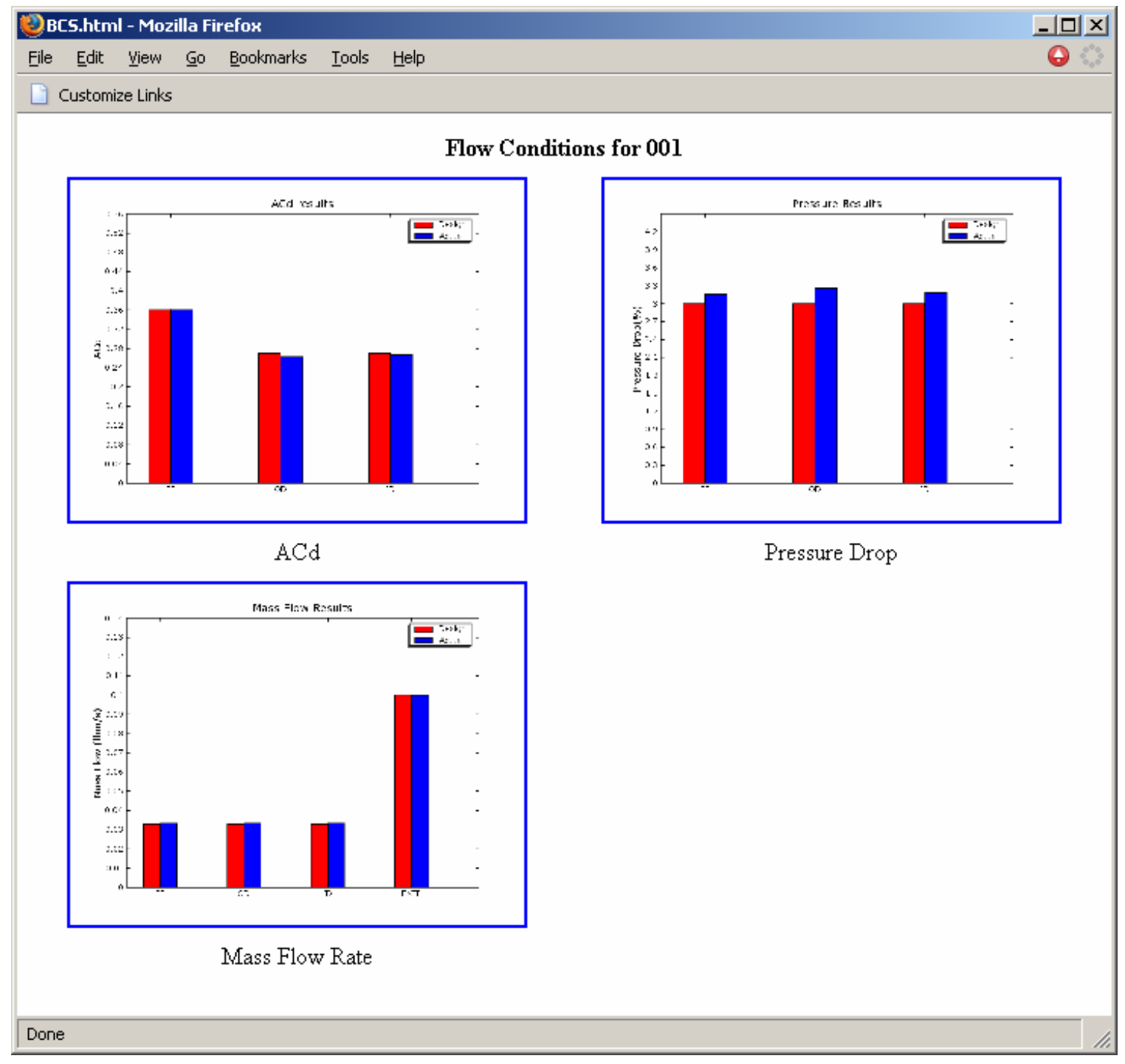

Flow Condition Section 


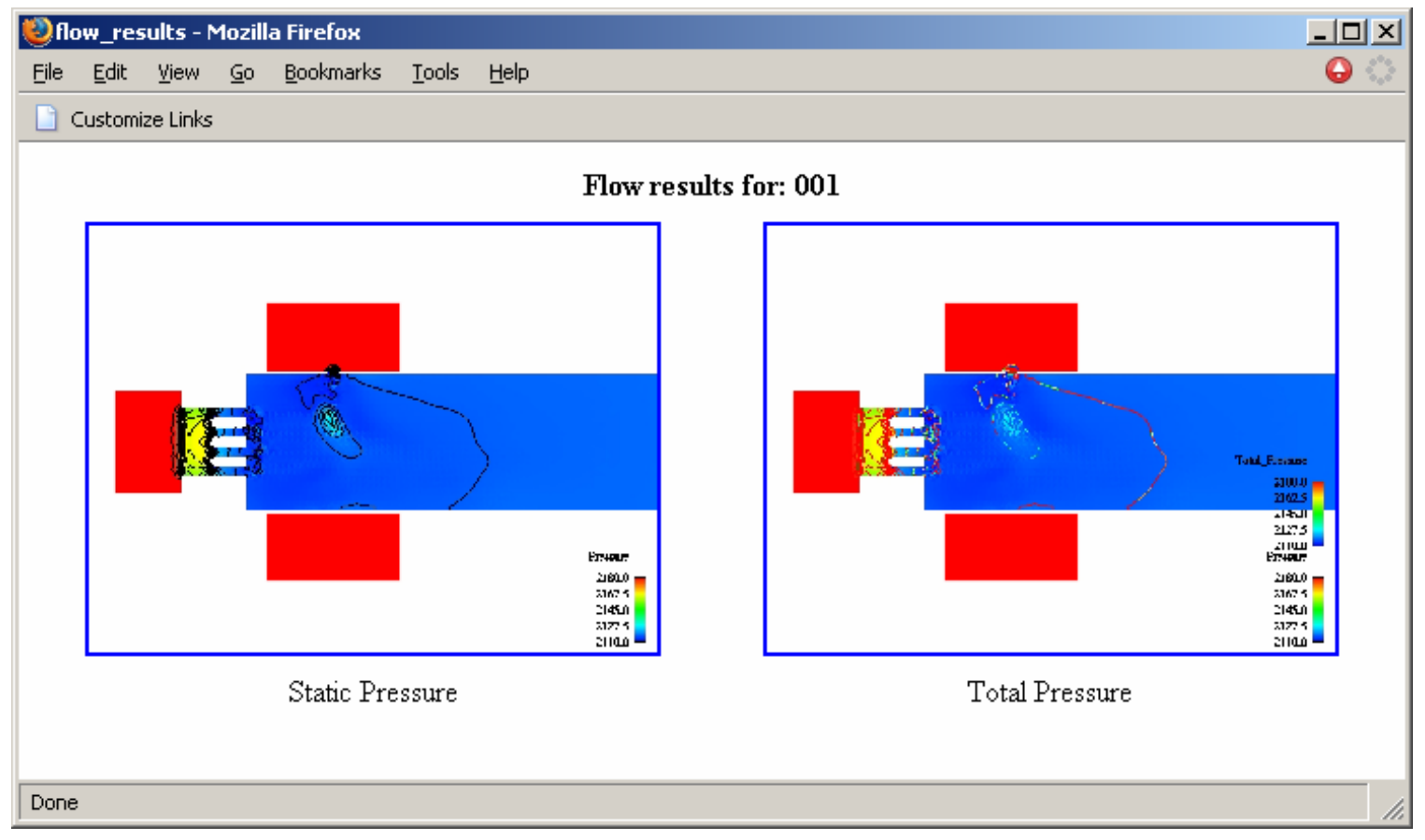

\section{Pressure Section}

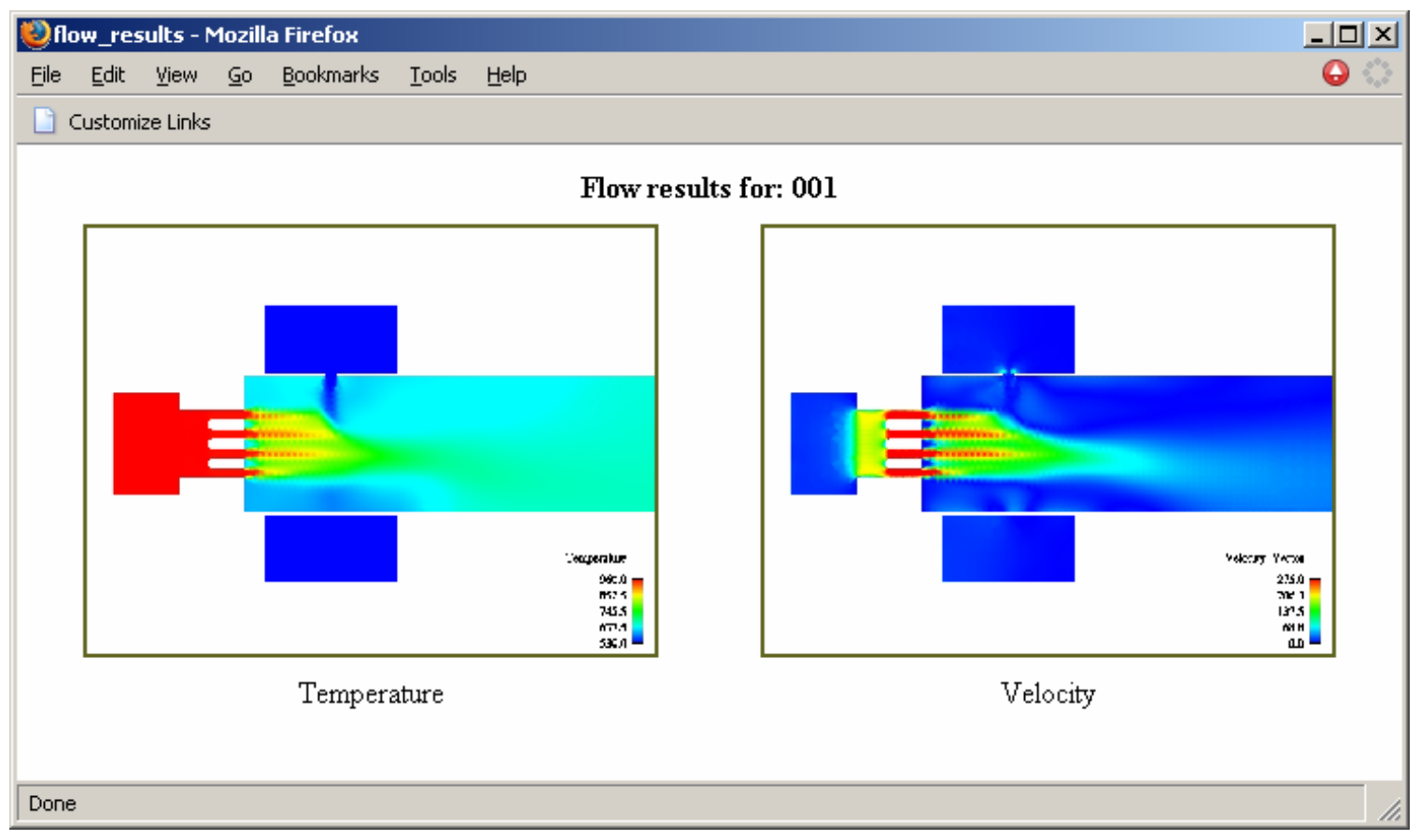

Flow Results Section 


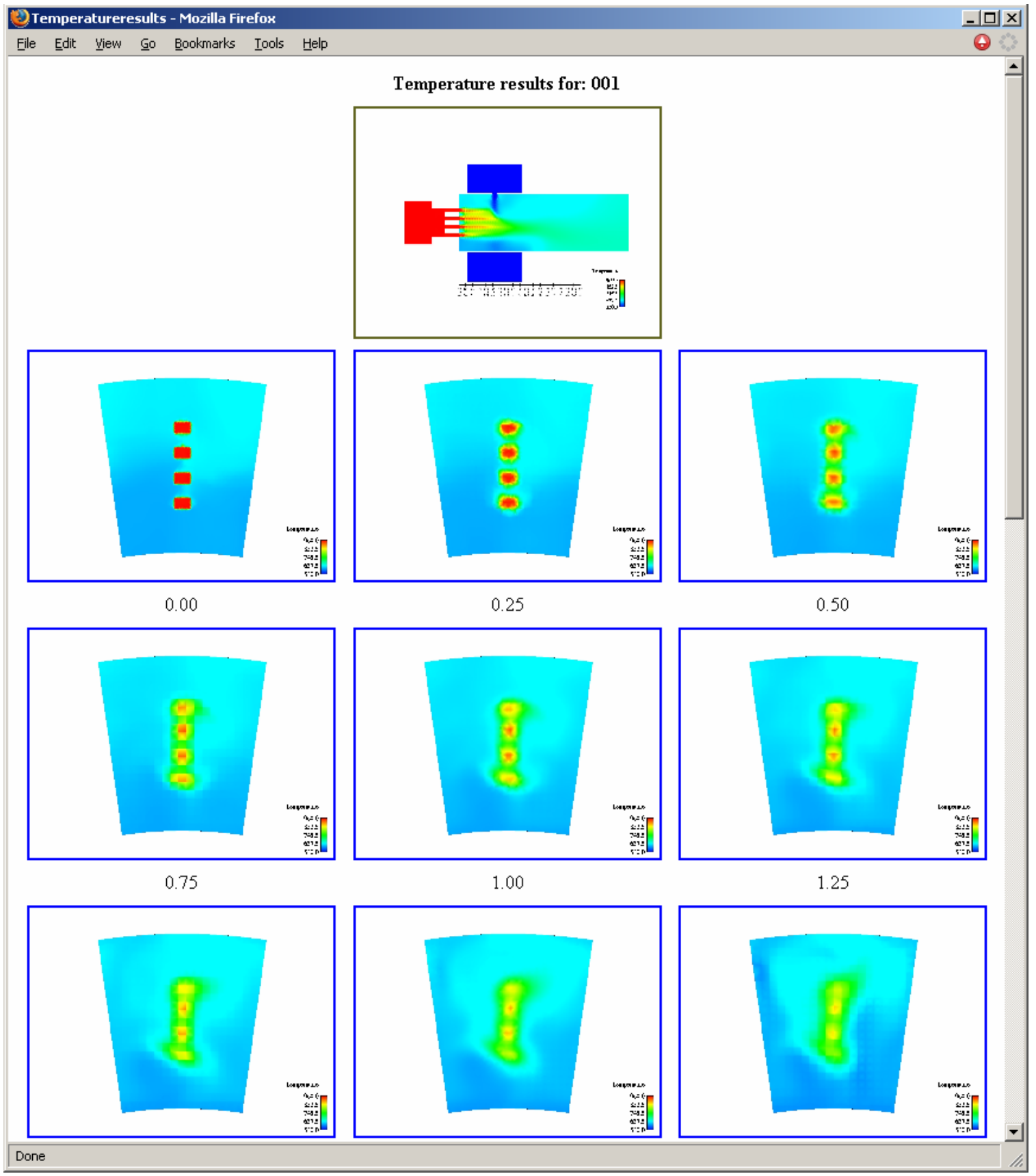




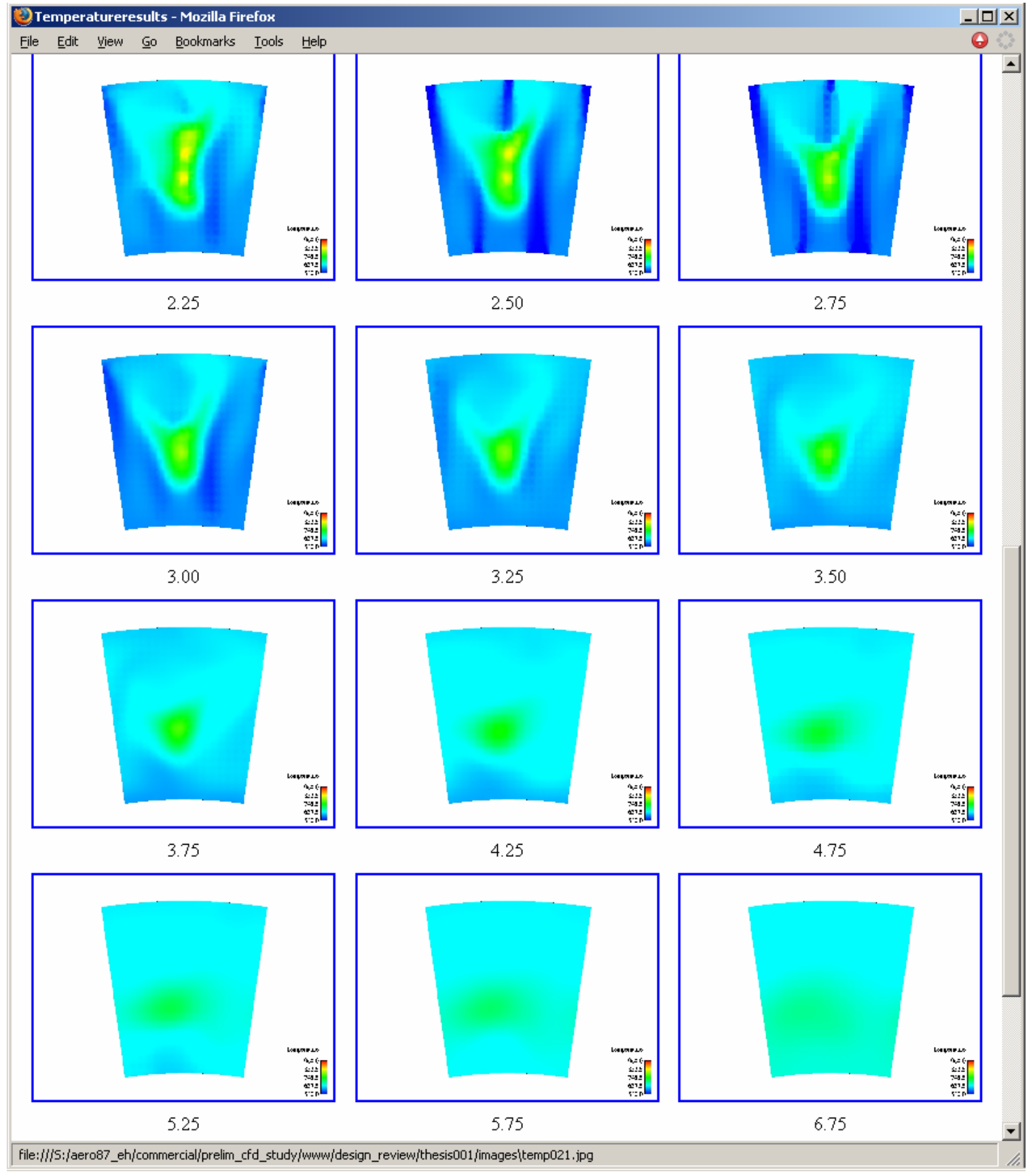




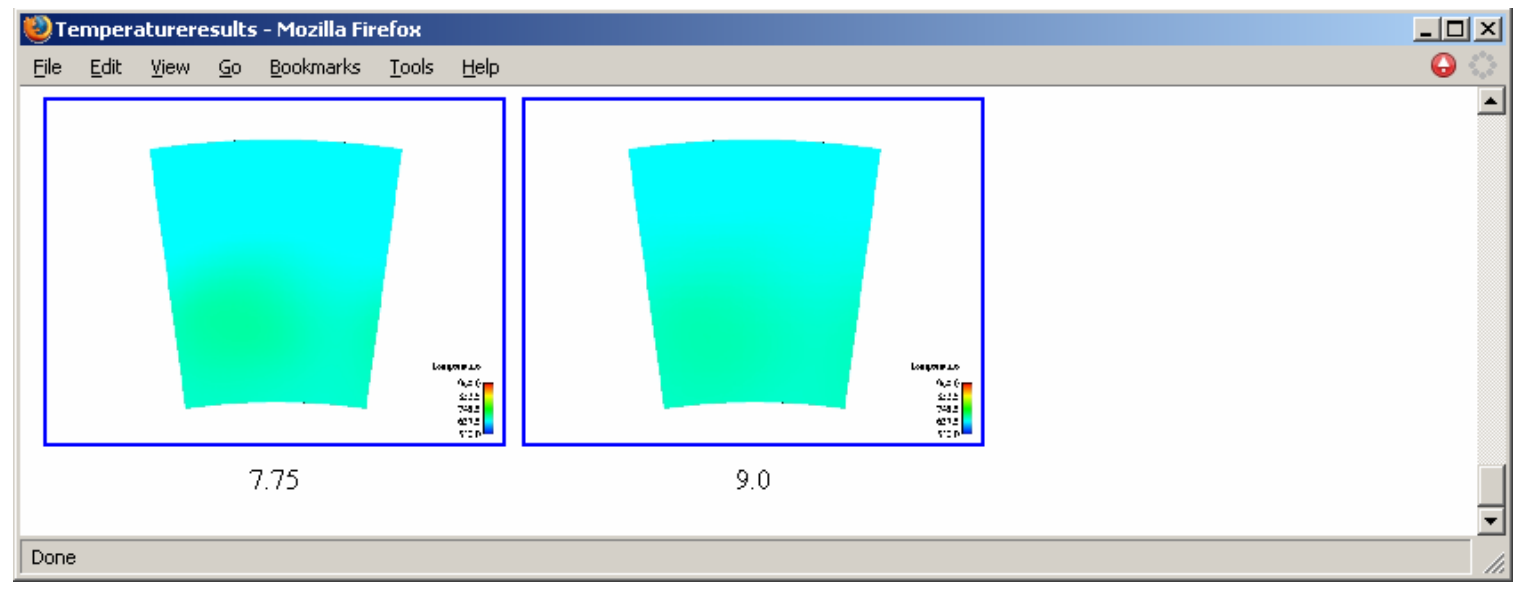

Temperature Results Section 


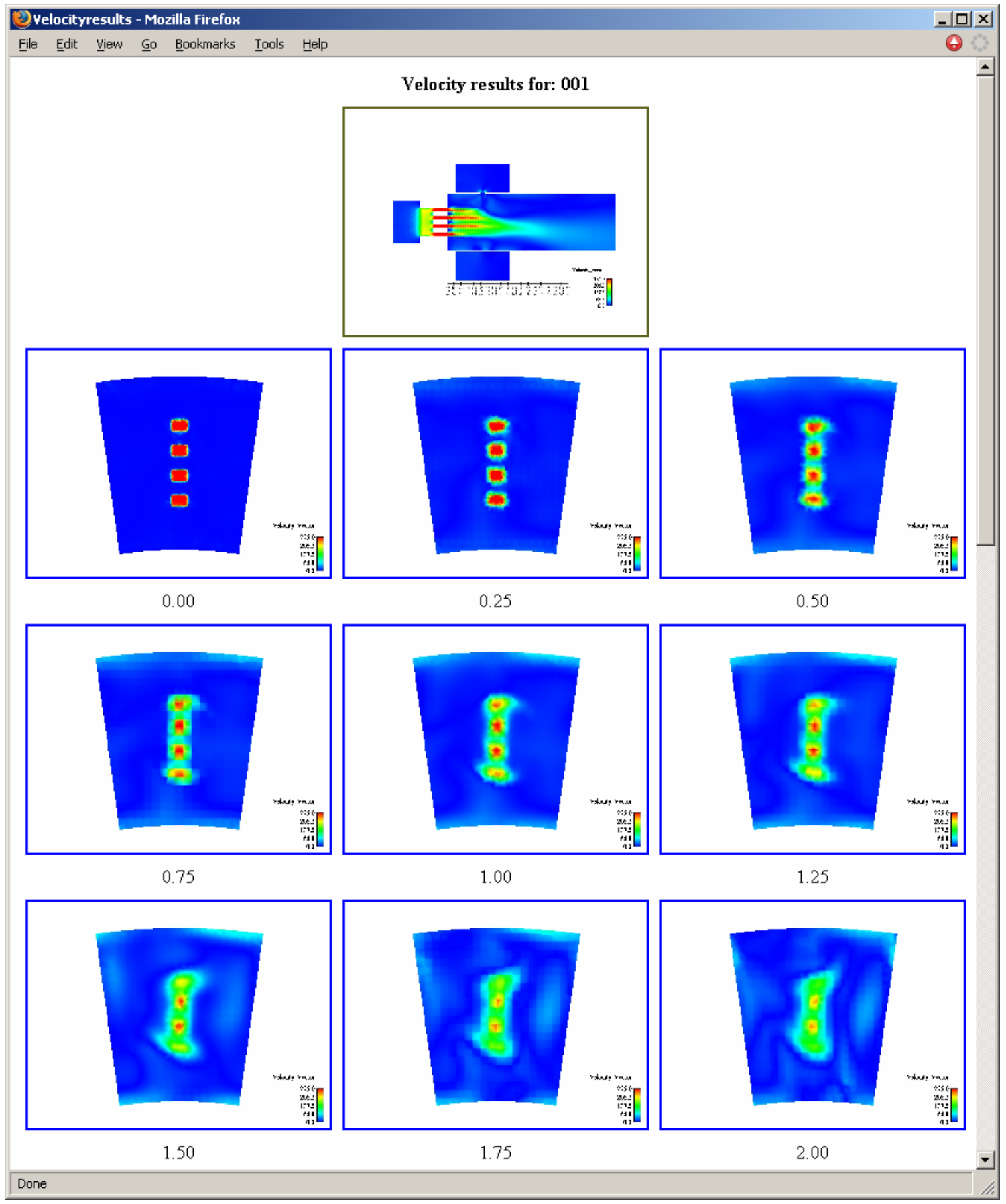




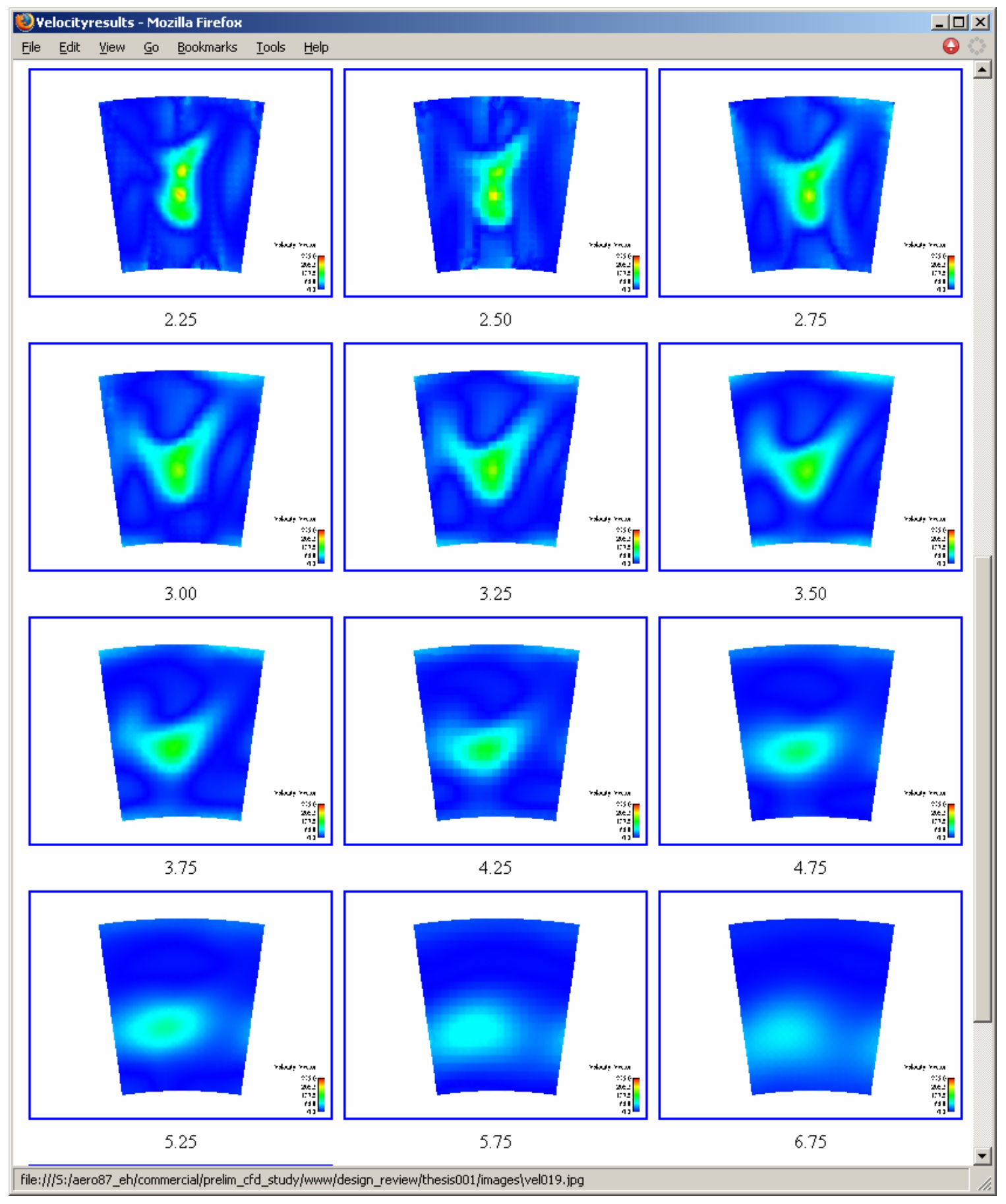




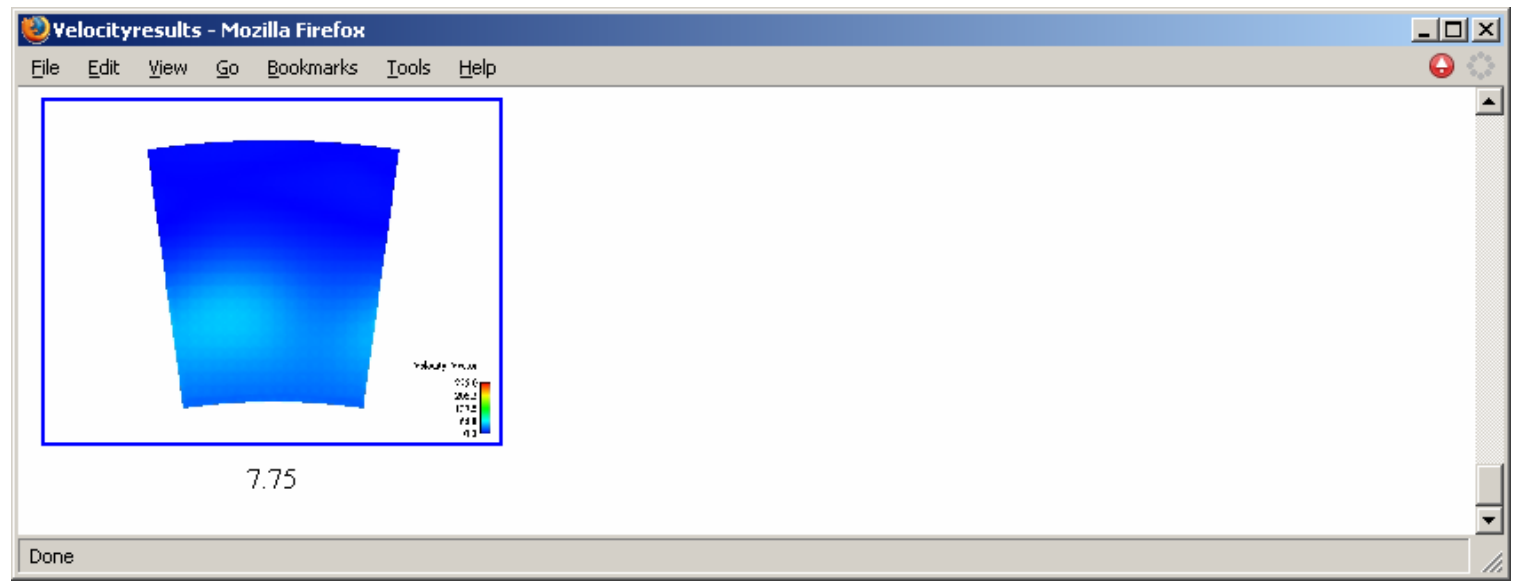

Velocity Results Section

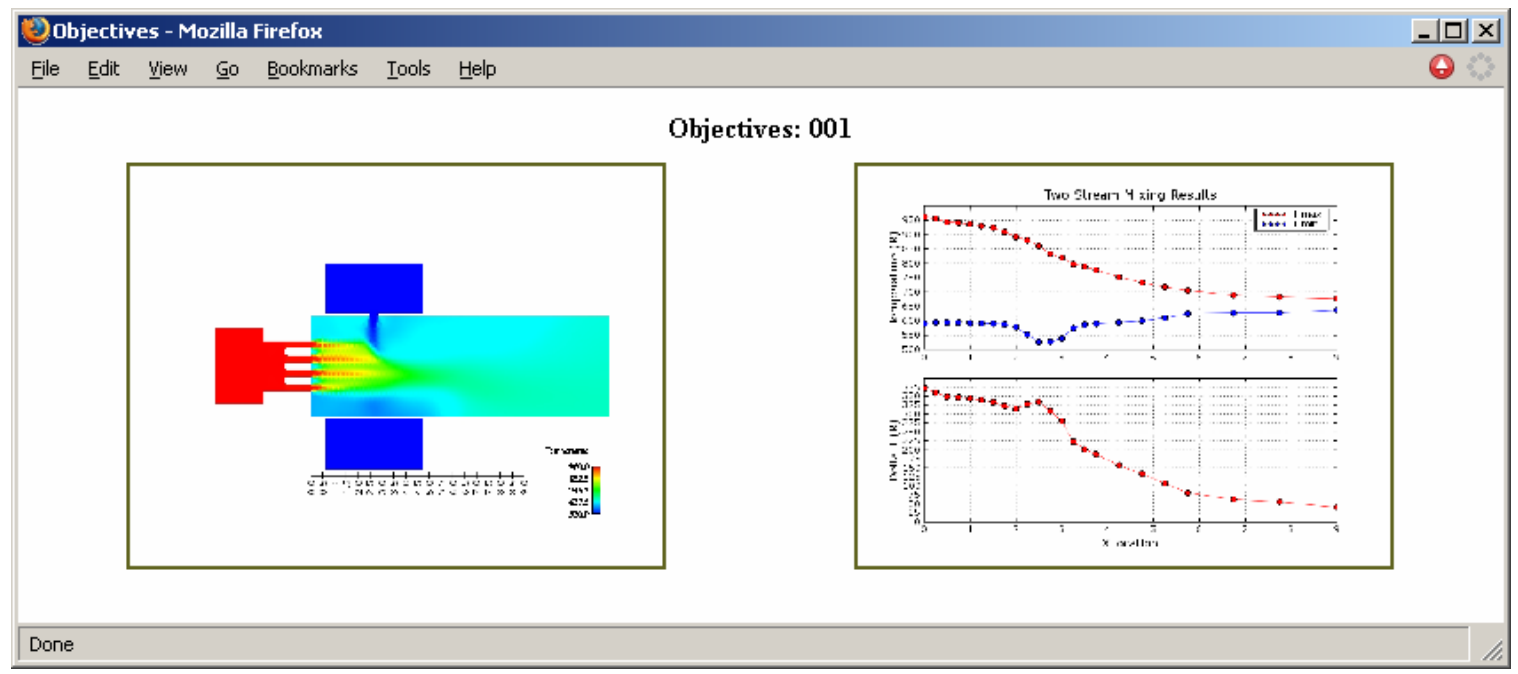

Objective Section 


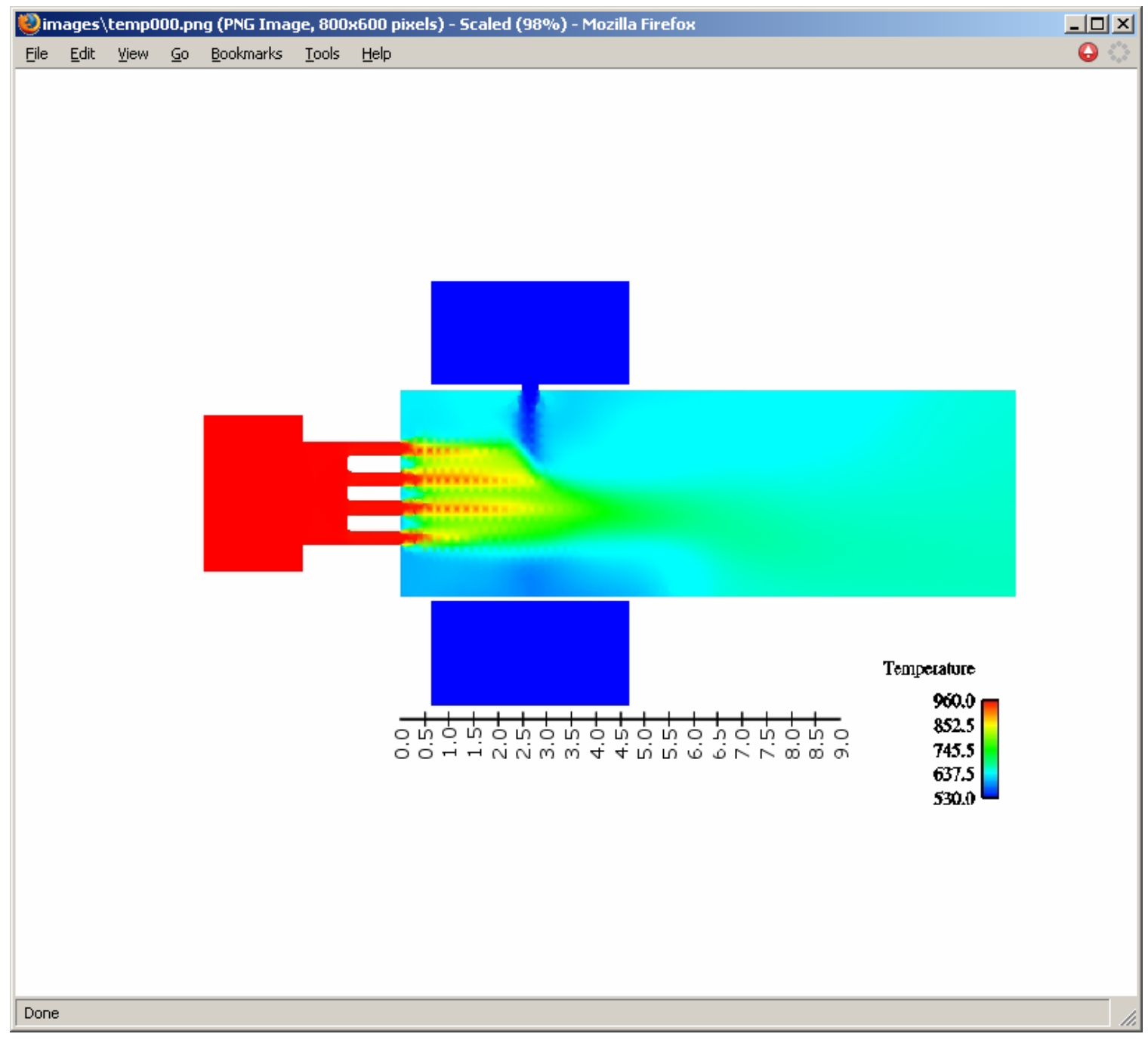

Temperature Plane Section 


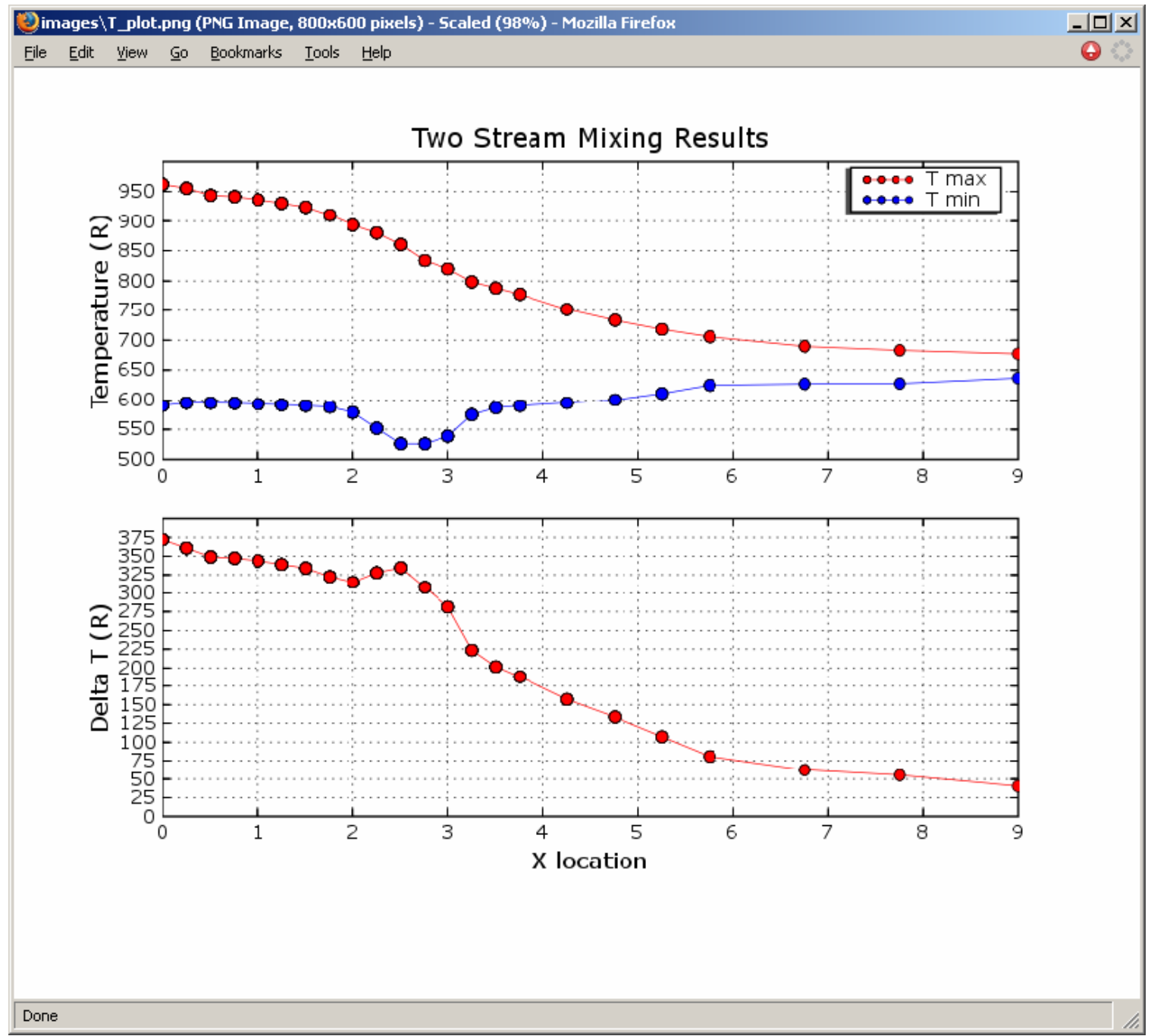

Mixing Results Section 